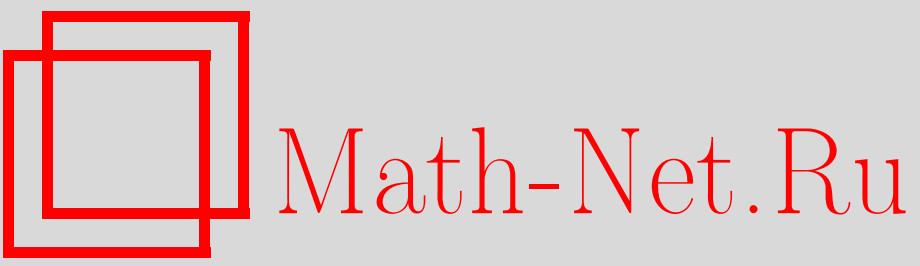

А. Д. Коршунов, Монотонные булевы функции, УМН, 2003, том 58, выпуск 5, 89-162

DOI: https://doi.org/10.4213/rm667

Использование Общероссийского математического портала Math-Net.Ru подразумевает, что вы прочитали и согласны с пользовательским соглашением

http://www.mathnet.ru/rus/agreement

Параметры загрузки:

IP: 54.162 .127 .20

26 апреля 2023 г., $11: 17: 21$ 


\title{
МОНОТОННЫЕ БУЛЕВЫ ФУНКЦИИ
}

\author{
А. Д. Коршунов
}

\begin{abstract}
Монотонные булевы функции являются важным объектом в дискретной математике и математической кибернетике. Тематика, связанная с такими функциями, активно исследуется в течение нескольких десятилетий. Получено значительное число результатов. Опубликовано большое число статей. Однако до сих пор нет ни монограбфии, ни обзорной статьи, где достаточно полно были бы освещены результаты исследований по монотонным булевым функциям.

Цель обзора - изложение основных результатов по монотонным булевым функциям, полученных за последние пятьдесят лет.

Библиографофия: 356 названий.
\end{abstract}

\section{СОДЕРЖАНИЕ}

Введение . . . . . . . 90

Глава 1. Число и структура монотонных булевых функций $\ldots . \ldots \ldots \ldots . .92$

$\S 1.1$. Число монотонных булевых функций ......................... 92

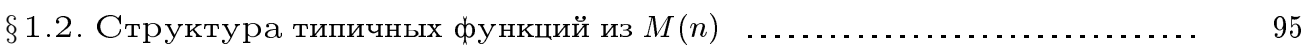

$\S 1.3$. Функции из $M(n)$ с фиксированным числом нижних единиц $\ldots . . . . . .996$

$\S 1.4$. Замкнутые классы монотонных булевых функций $\ldots \ldots \ldots \ldots \ldots \ldots . . .297$

$\S 1.5$. Структура типичных функций из замкнутых классов . ........... 100

$\S 1.6$. Еще одно множество монотонных булевых функций $\ldots . . . . . . . . . . .101$

$\S 1.7$. Монотонные булевы функции и шпернеровы семейства .......... 104

$\S 1.8$. Развитие метода. Другие результаты ......................... 105

Глава 2. Сложность вычисления (реализации) монотонных булевых функ-

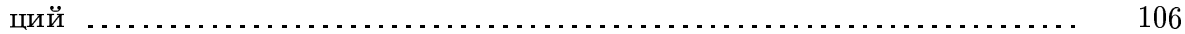

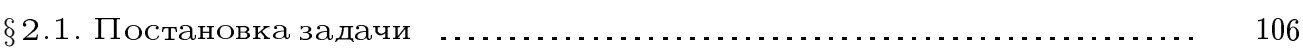

$\S 2.2$. Сложность булевых схем …................................ 108

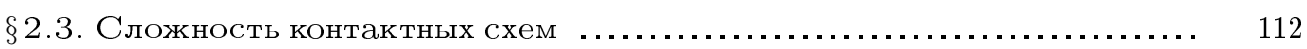

$\S 2.4$. Сложность вычисления и задания монотонных булевых функций из замкнутых классов ............................................. 114

$\S 2.5$. Симметрические монотонные булевы функции $\ldots \ldots \ldots \ldots \ldots \ldots \ldots \ldots . . \ldots \ldots$

$\S 2.6$. Влияние отрицаний на сложность вычислений монотонных булевых

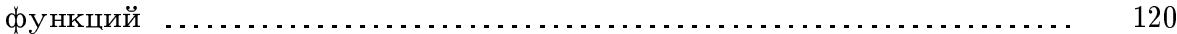


$\S 2.7$. Нижние оценки сложности схем, вычисляющих конкретные монотонные булевы функции . .................................... 121

$\S 2.8$. Высокие нижние оценки. Методы Разборова и Андреева ......... 125

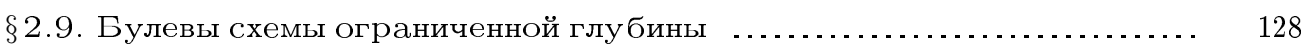

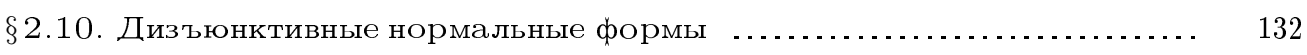

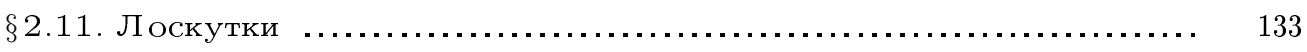

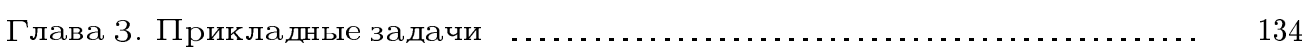

§3.1. Расшифровка монотонных булевых функций …............... 134

$\S 3.2$. Максимальные верхние нули монотонных булевых функций $\ldots . . . . .137$

$\S 3.3$. Пороговые монотонные булевы функции и системы линейных нера-

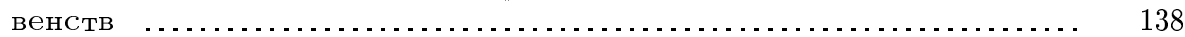

$\S 3.4$. Тесты и монотонные булевы функции ........................... 140

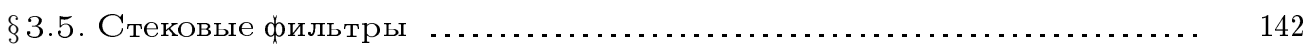

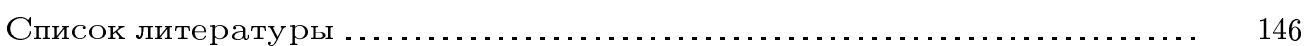

\section{Введение}

Булева функция - функция, переменные которой, как и сама функция, принимают значения 0 и 1 . Булевы функции являются одним из основных объектов дискретной математики. Они возникли при постановке логических задач и названы по имени Дж. Буля, положившего начало их применению в математической логике.

Позднее выяснилось, что язык булевых функций удобен для описания функционирования некоторых дискретных управляющих систем таких, как контактные схемы, булевы схемы (частный вид схем из функциональных элементов), логические сети и др. Такие управляющие системы представляют собой весьма содержательный объект с математической точки зрения.

В множестве всех булевых функций известны следуюшие классы функций: линейных, монотонных, пороговых, симметрических и некоторых других.

Среди этих классов значительньй интерес представляет класс монотонных булевых функций. Такой интерес объясняется несколькими причинами. Укажем некоторые из них.

Во-первых, класс монотонных булевых функций является наиболее мошным среди перечисленных классов и в то же время его структура сильно отличается от структуры множества всех булевых функций.

Во-вторых, класс монотонных булевых функций является предполным в множестве всех булевых функций, т.е. не существует замкнутого (относительно суперпозиции) класса булевых функций, содержащего все монотонные функции и отличного от класса монотонных булевых функций и множества всех булевых функций.

В-третьих, на язьке монотонных булевых функций ставятся и решаются разнообразные дискретные экстремальные задачи.

Работа выполнена при финансовой поддержке Российского фонда фундаментальных исследований (грант № 02-01-00939). 
Тематика, связанная с монотонными булевыми функциями, активно изучается в течение нескольких десятилетий. Получено много результатов. Опубликовано несколько сот статей. Отдельные результаты освешены в монографиях [61], [62], [187], [190], [209], [210], [226], [268], [329], [338], [55] и обзорах [20], [30], [57], [58], [85], [101], [133], [159], [160], [186], [249], [267], [351]. Однако до сих пор нет ни монографии, ни обзорной статьи, где достаточно полно были бы изложены результаты исследований по монотонньмм булевьгм функциям.

Цель настоящего обзора состоит в освешении основных результатов по монотонным булевьм функциям, полученных за последние пятьдесят лет.

Следует отметить, что в обзоре нет сведений о диссертациях, препринтах, отчетах, тезисах и статьях в электронном виде, поскольку содержашиеся в них результаты, как правило, опубликованы в более доступных изданиях. В нем также отсутствует информация о статьях с чисто логическим содержанием.

На протяжении всего обзора используются следующие понятия и обозначения.

Пусть $\widetilde{\alpha}=\left(\alpha_{1}, \ldots, \alpha_{n}\right)$ и $\widetilde{\beta}=\left(\beta_{1}, \ldots, \beta_{n}\right)$ - различные двоичные наборы длины $n$. Говорят, что набор $\widetilde{\alpha}$ предшествует набору (меньше набора) $\widetilde{\beta}$ (обозначение: $\widetilde{\alpha} \prec \widetilde{\beta})$, если $\alpha_{i} \leqslant \beta_{i}$ при каждом $i, 1 \leqslant i \leqslant n$.

Булева функция $f\left(x_{1}, \ldots, x_{n}\right)$ называется монотонной, если $f(\widetilde{\alpha}) \leqslant f(\widetilde{\beta})$ на любых двоичных наборах $\widetilde{\alpha}=\left(\alpha_{1}, \ldots, \alpha_{n}\right)$ и $\widetilde{\beta}=\left(\beta_{1}, \ldots, \beta_{n}\right)$ таких, что $\widetilde{\alpha} \prec \widetilde{\beta}$.

Множество монотонных булевых функций от $n$ фиксированных переменных обозначается через $M(n)$.

Двоичный набор $\widetilde{\alpha}$ длины $n$ называется нижней единичей функции $f \in M(n)$, если $f(\widetilde{\alpha})=1$ и $f(\widetilde{\beta})=0$ на любом таком двоичном наборе $\widetilde{\beta}$ длины $n$, что $\widetilde{\beta} \prec \widetilde{\alpha}$.

Двоичный набор $\widetilde{\alpha}$ длины $n$ назьвается верхним нулем функции $f \in M(n)$, если $f(\widetilde{\alpha})=0$ и $f(\widetilde{\beta})=1$ на любом таком двоичном наборе $\widetilde{\beta}$ длины $n$, что $\widetilde{\alpha} \prec \widetilde{\beta}$.

Число единиц в двоичном наборе назьвается весом набора. Через $E^{n}$ обозначается $n$-мерный булев куб, т.е. неориентированньй граф с $2^{n}$ вершинами, которые помечены двоичньми наборами длины $n$. В этом графе две вершины, помеченные наборами $\widetilde{\alpha}=\left(\alpha_{1}, \ldots, \alpha_{n}\right)$ и $\widetilde{\beta}=\left(\beta_{1}, \ldots, \beta_{n}\right)$, смежны (соединены ребром) тогда и только тогда, когда расстояние Хемминга $\rho(\widetilde{\alpha}, \widetilde{\beta})=\sum_{i=1}^{n}\left|\alpha_{i}-\beta_{i}\right|=1$.

Множество вершин в $E^{n}$, помеченных наборами веса $k, 0 \leqslant k \leqslant n$, называется $k$-м слоем в $E^{n}$ и обозначается через $E^{n, k}$.

Через $\lfloor x\rfloor$ обозначается наибольшее натуральное число, не превосходящее действительное число $x$, а через $\lceil x\rceil$ - минимальное натуральное число, не меньшее $x$.

Пусть функции $f(n)$ и $g(n)$ принимают положительные значения при любом натуральном $n$. Тогда запись $f(n)=O(g(n))(f(n) \leqslant O(g(n)))$ означает: сушествует такая константа $c>0$, что $f(n)<c \cdot g(n)$ при любом $n \geqslant 1$. Запись $f(n)=\Theta(g(n))$ означает: сушествуют константы $c_{1}>0$ и $c_{2}>0$ такие, что $c_{1} g(n) \leqslant f(n) \leqslant c_{2} g(n)$ при любом $n \geqslant 1$. Запись $f(n) \sim g(n)$ означает, что $\lim _{n \rightarrow \infty}(f(n) / g(n))=1$. Запись $f(n)=\Omega(g(n))$ означает сушествование константы $c>0$ такой, что $f(n)>c \cdot g(n)$ при любом $n$. 


\section{Глава 1.}

\section{Число и структура монотонных булевых функций}

\section{§1.1. Число монотонных булевых функций}

Задача о числе монотонных булевых функций от $n$ переменньх известна давно. Первоначально она рассматривалась Р. Дедекиндом на алгебраическом языке как задача о числе свободных дистрибутивных структур с $n$ образуюшими [47]. Позднее эта задача также стала известна как комбинаторная задача о числе шеернеровых семейств, заданных на $n$-элементном множестве [290]. Краткие сведения о шерернеровых семействах будут даны в $\S 1.7$.

Длительное время задача о нахождении $|M(n)|$ - числа монотонных булевых функций от $n$ переменных - не рассматривалась. На нее было обращено внимание только в 40-х годах. В настоящее время известно, что $|M(1)|=3,|M(2)|=6,|M(3)|=20$, $|M(4)|=168,|M(5)|=7581$ [40], [41], [174], $|M(6)|=7828354$ [46], [174], [316], $|M(7)|=2414682040998$ [16], [17], [40], [109], $|M(8)|=56130437228687557907788$ [333].

Поскольку число функций из $M(n)$ быстро возрастает с ростом $n$ (например, $\left.|M(9)|>10^{42}\right)$, возможность вычисления $|M(n)|$ при $n>8$ становится проблематичной.

Конечно, при рассмотрении задачи о числе монотонных булевых функций от $n$ переменных возникает желание найти точные формулы. Однако, по всей видимости, постановка задачи о нахождении точных формул для числа объектов, обладаюших заданными свойствами, во многих случаях требует уточнения, ибо произвольные формулы можно получить довольно просто. В самом деле, пусть в множестве объектов из конечного множества требуется найти число объектов, обладающих нетривиальными предписанными свойствами. Это число можно найти следуюшим образом. Сначала все объекты множества подходящим способом нумеруются. Затем они перебираются в порядке нумерации. Наконец, про каждый очередной объект выясняется, обладает ли он заданньми свойствами. Эту процедуру, как правило, можно записать в виде формулы. Приведем пример.

Пусть $\pi(n)$ обозначает число простых чисел, не превосходяших натурального числа $n$. Тогда при любом $n \geqslant 4$ имеем

$$
\pi(n)=2+\sum_{s=4}^{n} \operatorname{sign} \prod_{k=2}^{\sqrt{s}}\left(\frac{s}{k}-\left\lfloor\frac{s}{k}\right\rfloor\right) .
$$

Эта формула проста. Однако не ясно, как можно извлечь из нее информацию о величине $\pi(n)$.

Для числа монотонных булевых функций от $n$ переменных известна следуюшая формула:

$$
|M(n)|=\sum_{k=1}^{2^{2^{n}}} \prod_{j=1}^{2^{n}-1} \prod_{i=0}^{j-1}\left\{1-b_{i}^{k} b_{j}^{k} \prod_{m=0}^{\log _{2} i}\left(1-b_{m}^{i}+b_{m}^{i} b_{m}^{j}\right)\right\}
$$


где $b_{s}^{r}=\left\lfloor r 2^{-s}\right\rfloor-2\left\lfloor r 2^{-s-1}\right\rfloor$. Эта формула предложена А. Киселевичем [134] и по сушеству является формульной записью алгоритма перебора по всем булевым функциям от $n$ переменных и проверкой их на монотонность.

Более сложная точная формула для $|M(n)|$ имеется в [83]. Наряду с точными формулами для $|M(n)|$ известны рекуррентные формулы [15], [65], [109], [257].

Нахождением нижних и верхних оценок для $|M(n)|$ в общем случае занимались в течение длительного времени. Очевидной является нижняя оценка

$$
|M(n)|>2^{\left(\begin{array}{c}
n \\
\lfloor n / 2\rfloor
\end{array}\right),}
$$

где правая часть равна числу монотонных булевых функций из $M(n)$, все нижние единицы которых расположены в $E^{n,\lfloor n / 2\rfloor}$.

Первые нетривиальные верхние оценки были получены в [78], [316], [340]. Например, оценка

$$
\left.|M(n)|<n^{(\lfloor n / 2\rfloor}\right)+2
$$

содержится в статье [78]. Более точная верхняя оценка для $|M(n)|$ была получена В. К. Коробковым [144]-[146]. Эта оценка имеет вид

$$
|M(n)|<2^{5\left(\begin{array}{c}
n \\
\lfloor n / 2\rfloor
\end{array}\right)} .
$$

Ж. Ансель в [96] понизил эту оценку до следующей:

$$
|M(n)|<3^{\left(\begin{array}{c}
n \\
\lfloor n / 2\rfloor
\end{array}\right)}
$$

(см. также [277]). Дальнейшее качественное продвижение в решении задачи о числе монотонных булевых функций от $n$ переменных получили Д. Клейтман и Дж. Марковский [137], [139]. Используя подход Ж. Анселя, они доказали, что

$$
\log _{2}|M(n)| \sim\left(\begin{array}{c}
n \\
\lfloor n / 2\rfloor
\end{array}\right)\left(1+O\left(\frac{\log n}{n^{1 / 2}}\right)\right)
$$

Усиление этого результата имеется в работе [223], в которой показано, что

$$
\log _{2}|M(n)| \sim\left(\begin{array}{c}
n \\
\lfloor n / 2\rfloor
\end{array}\right)\left(1+O\left(\frac{(\log n)^{3 / 2}}{n^{1 / 2}}\right)\right) .
$$

K этому же направлению относятся работы [46], [107], [108], [179].

Асимптотики для $|M(n)|$ были получены автором настоящей статьи в 1977 году [151] (для нечетных $n$ в [151] имеется опечатка). Полное доказательство этого результата было опубликовано в [152]. Оказалось, что в зависимости от четности $n$ асимптотические формулы различны. Они имеют следуюший вид. 
Теорема 1.1. При четном $n \rightarrow \infty$

$$
|M(n)| \sim 2^{\left(\begin{array}{c}
n \\
n / 2
\end{array}\right)} \exp \left\{\left(\begin{array}{c}
n \\
n / 2-1
\end{array}\right)\left(2^{-n / 2}+n^{2} 2^{-n-5}-n 2^{-n-4}\right)\right\}
$$

a при нечетном $n \rightarrow \infty$

$$
\begin{gathered}
|M(n)| \sim 2^{\left(\begin{array}{c}
n \\
(n-1) / 2
\end{array}\right)+1} \exp \left\{\left(\begin{array}{c}
n \\
(n-3) / 2
\end{array}\right)\left(2^{-(n+3) / 2}-n^{2} 2^{-n-6}-n 2^{-n-3}\right)\right. \\
\left.+\left(\begin{array}{c}
n \\
(n-1) / 2
\end{array}\right)\left(2^{-(n+1) / 2}+n^{2} 2^{-n-4}\right)\right\} .
\end{gathered}
$$

Кратко поясним основную идею доказательства теоремы 1.1. Множество всех функций из $M(n)$ разбивается на непересекающиеся классы $M(n, k), 0 \leqslant k \leqslant n$. Функция $f \in M(n)$ включается в $k$-й класс, если существует набор $\widetilde{\alpha} \in E^{n, k}$ такой, что $f(\widetilde{\alpha})=1$ и $f(\widetilde{\beta})=0$ при любом $\widetilde{\beta}$, в котором число единиц меньше $k$. Первый этап доказательства заключается в установлении следуюшего утверждения.

ТЕОРема 1.2. При любом $k<\lfloor n / 2\rfloor u n \rightarrow \infty$

$$
|M(n, k-1)|=o(|M(n, k)|)
$$

Из этой теоремы следует, что при четных $n$ нижние единицы почти каждой функции из $M(n)$ могут располагаться только в $E^{n, n / 2-1}, E^{n, n / 2}$ и $E^{n, n / 2+1}$, а при нечетных $n-$ в $E^{n,(n-3) / 2}, E^{n,(n-1) / 2}, E^{n,(n+1) / 2}$ и $E^{n,(n+3) / 2}$.

Перед доказательством теоремы 1.2 предварительно изучалась следующая вспомогательная задача. Пусть $\widetilde{\alpha}-$ произвольная вершина $k$-го слоя. Множество всех вершин $\widetilde{\beta}$ из $(k+1)$-го слоя таких, что $\widetilde{\beta} \succ \widetilde{\alpha}$, называется 1 -тенью для $\widetilde{\alpha}$. Пусть $A-$ произвольное подмножество вершин $k$-го слоя. Множество всех вершин $\widetilde{\beta}$ из $(k+1)$-го слоя таких, что $\widetilde{\beta}$ принадлежит 1-тени некоторой вершины из $A$, называется 1 -тенью множества $A$ и обозначается через $T^{1}(A)$. Далее, пусть $R(n, k, r, t)$ обозначает совокупность $r$-элементных подмножеств $A$ из $E^{n, k}$ таких, что $T^{1}(A)=t$. Задача состоит в нахождении величины $|R(n, k, r, t)|$ при всех допустимых значениях параметров $n, k, r, t$. Мы, не зная точного значения этой величины, установили для нее лишш приемлемые верхние оценки. При различных $n, k, r, t$ найденные оценки различны, но во многих случаях они имеют вид

$$
|R(n, k, r, t)|=o\left(2^{t}\right)
$$

Эти оценки существенным образом используются при доказательстве теоремы 1.2. Затем проводятся дополнительные исследования, позволяюшие установить ряд свойств, которьми обладают почти все функции из $M(n)$. Наконец, для числа таких функций находятся асимптотические формулы. 


\section{$\S 1.2$. Структура типичных функций из $M(n)$}

Пусть $A=\left\{\widetilde{\alpha}_{1}, \ldots, \widetilde{\alpha}_{r}\right\}$ - произвольное $r$-элементное множество вершин из $E^{n, k}$. Множество $A$ разобьем на связки: вершины $\widetilde{\alpha}_{i}$ и $\widetilde{\alpha}_{j}$ включаются в одну связку тогда и только тогда, когда в $A$ имеются вершины $\widetilde{\alpha}_{s_{1}}, \ldots, \widetilde{\alpha}_{s_{v}}$ такие, что расстояние Хемминга $\rho\left(\widetilde{\alpha}_{i}, \widetilde{\alpha}_{s_{1}}\right)=\rho\left(\widetilde{\alpha}_{s_{v}}, \widetilde{\alpha}_{j}\right)=2$ и $\rho\left(\widetilde{\alpha}_{s_{w}}, \widetilde{\alpha}_{s_{w+1}}\right)=2$ при любом $w, 1 \leqslant w \leqslant s-1$.

При четном $n$ обозначим через $M_{0}(n)$ множество функций $f \in M(n)$ таких, что

1 ) все нижние единицы функции $f$ находятся в $E^{n, n / 2-1}, E^{n, n / 2}$ и $E^{n, n / 2+1}$;

2) в $E^{n, n / 2}$ функция $f$ имеет не менее $\frac{1}{2}\left(\begin{array}{c}n \\ n / 2\end{array}\right)-n 2^{n / 2}$ и не более $\frac{1}{2}\left(\begin{array}{c}n \\ n / 2\end{array}\right)+n 2^{n / 2}$ нижних единиц;

3 ) функция $f$ имеет не более $2^{n / 2}$ нижних единиц в $E^{n, n / 2-1}$, множество этих единиц состоит из одноэлементных и двухэлементных связок и число двухэлементных связок не превосходит $n^{4}$;

4) функция $f$ имеет не более $2^{n / 2}$ нижних единиц в $E^{n, n / 2+1}$, множество этих единиц состоит из одноэлементных и двухэлементных связок и число двухэлементных связок не превосходит $n^{4}$;

5) функшия $f$ равна 1 во всех вершинах из $E^{n, n / 2+2}, \ldots, E^{n, n}$.

В [152] доказано, что почти все функции из $M(n)$ принадлежат множеству $M_{0}(n)$, т.е. при $n \rightarrow \infty$ выполнено соотношение

$$
\left|M_{0}(n)\right| \sim|M(n)|
$$

При нечетном $n$ обозначим через $M_{1}(n)$ множество функций $f \in M(n)$ таких, что

$1)$ все нижние единицы функщии $f$ находятся в $E^{n,(n-3) / 2}, E^{n,(n-1) / 2}$ и $E^{n,(n+1) / 2}$;

$2)$ в $E^{n,(n-1) / 2}$ функция $f$ имеет не менее $\frac{1}{2}\left(\begin{array}{c}n \\ (n-1) / 2\end{array}\right)-n 2^{n / 2}$ и не более $\frac{1}{2}\left(\begin{array}{c}n \\ (n-1) / 2\end{array}\right)+$ $n 2^{n / 2}$ нижних единиц;

$3)$ функция $f$ имеет не более $2^{n / 2}$ нижних единиц в $E^{n,(n-3) / 2}$, множество этих единиц состоит из одноэлементных и двухэлементных связок и число двухэлементных связок не превосходит $n^{4}$;

4) функция $f$ имеет не более $2^{n / 2}$ нижних единиц в $E^{n,(n+1) / 2}$, множество этих единиц состоит из одноэлементных и двухэлементных связок и число двухэлементных связок не превосходит $n^{4}$;

$5)$ функция $f$ равна 1 во всех вершинах из $E^{n,(n+3) / 2}, \ldots, E^{n, n}$.

Далее при нечетном $n$ обозначим через $M_{2}(n)$ множество функций $f \in M(n)$ таких, что

1) все нижние единицы функции $f$ находятся в $E^{n,(n-1) / 2}, E^{n,(n+1) / 2}$ и $E^{n,(n+3) / 2}$;

$2)$ в $E^{n,(n+1) / 2}$ функция $f$ имеет не менее $\frac{1}{2}\left(\begin{array}{c}n \\ (n+1) / 2\end{array}\right)-n 2^{n / 2}$ и не более $\frac{1}{2}\left(\begin{array}{c}n \\ (n+1) / 2\end{array}\right)+$ $n 2^{n / 2}$ нижних единиц;

3) функция $f$ имеет не более $2^{n / 2}$ нижних единиц в $E^{n,(n-1) / 2}$, множество этих единиц состоит из одноэлементных и двухэлементных связок и число двухэлементных связок не превосходит $n^{4}$;

4) функция $f$ имеет не более $2^{n / 2}$ нижних единиц в $E^{n,(n+3) / 2}$, множество этих единиц состоит из одноэлементных и двухэлементных связок и число двухэлементных связок не превосходит $n^{4}$;

5) функция $f$ равна 1 во всех вершинах из $E^{n,(n+5) / 2}, \ldots, E^{n, n}$. 
В [152] доказано, что при $n \rightarrow \infty$

$$
\left|M_{1}(n)\right|+\left|M_{2}(n)\right| \sim|M(n)|
$$

Асимптотические формулы для числа монотонных булевых функций от $n$ переменных и информация о строении типичных функщий из $M(n)$ включены в монографии [61], [62], [329], [55].

\section{$\S$ 1.3. Функции из $M(n)$ с фиксированным числом нижних единиц}

В предыдущем параграфе было отмечено, что почти все функции из $M(n)$ принадлежат множеству $M_{0}(n)$ при четном $n$ и множеству $M_{3}(n)=M_{1}(n) \cup M_{2}(n)$ при нечетном $n$. Теперь сформулируем утверждение о числе функций из $M_{0}(n)$ и $M_{3}(n)$ с наиболее вероятным числом нижних единиц.

При четном $n$ обозначим через $M_{0}(n, z)$ множество функций $f \in M_{0}(n)$ таких, что $f$ имеет $z$ нижних единиц.

Tеорема 1.3 [163]. Пусть $п$ четно и

$$
z_{0}=\left\lfloor\frac{1}{2}\left(\begin{array}{c}
n \\
n / 2
\end{array}\right)\left(1-\frac{n / 2-1}{2^{n / 2}}\right)\right\rfloor
$$

Тогда при любом $t,|t| \leqslant n 2^{n / 2}$, и $n \rightarrow \infty$ справедливо соотношение

$$
\left|M_{0}\left(n, z_{0}+t\right)\right| \sim \sqrt{2 /\left(\pi\left(\begin{array}{c}
n \\
n / 2
\end{array}\right)\right)}|M(n)| \exp \left(-2 t^{2} /\left(\begin{array}{c}
n \\
n / 2
\end{array}\right)\right) .
$$

При нечетном $n$ обозначим через $M_{3}(n, z)$ множество функций $f \in M_{3}(n)$ таких, что $f$ имеет $z$ нижних единищ.

TеOPeMa 1.4 [163]. Пусть $п$ четно и

$$
\begin{aligned}
z_{1}= & \frac{1}{2}\left(\begin{array}{c}
n \\
(n-1) / 2
\end{array}\right)-\left(\begin{array}{c}
n \\
(n-3) / 2
\end{array}\right)(n-1) 2^{-(n+1) / 2-3} \\
& \left.-\left(\begin{array}{c}
n \\
(n-1) / 2
\end{array}\right)(n-3) 2^{-(n+1) / 2-2}\right\rfloor .
\end{aligned}
$$

Тогда при любом $t,|t| \leqslant n 2^{n / 2}$, и $n \rightarrow \infty$ справедливо соотношение

$$
\left|M_{3}\left(n, z_{1}+t\right)\right| \sim \sqrt{2 /\left(\pi\left(\begin{array}{c}
n \\
(n-1) / 2
\end{array}\right)\right)}|M(n)| \exp \left\{-2 t^{2} /\left(\begin{array}{c}
n \\
(n-1) / 2
\end{array}\right)\right\}
$$

При четном $n$ положим

$$
M_{0}^{\prime}(n)=\bigcup_{|t| \leqslant n 2^{n / 2}} M_{0}\left(n, z_{0}+t\right)
$$


Тогда из теоремы 1.3 следует, что при $n \rightarrow \infty$

$$
\begin{aligned}
M_{0}^{\prime}(n) & \sim \sqrt{2 /\left(\pi\left(\begin{array}{c}
n \\
n / 2
\end{array}\right)\right)}|M(n)| \sum_{|t| \leqslant n 2^{n / 2}} \exp \left\{-2 t^{2} /\left(\begin{array}{c}
n \\
n / 2
\end{array}\right)\right\} \\
& \sim \sqrt{2 /\left(\pi\left(\begin{array}{c}
n \\
n / 2
\end{array}\right)\right)}|M(n)| \int_{-n 2^{n / 2}}^{n 2^{n / 2}} \exp \left\{-2 x^{2} /\left(\begin{array}{c}
n \\
n / 2
\end{array}\right)\right\} d x \\
& \sim \sqrt{2 /\left(\pi\left(\begin{array}{c}
n \\
n / 2
\end{array}\right)\right)}|M(n)| \int_{-\infty}^{\infty} \exp \left\{-2 x^{2} /\left(\begin{array}{c}
n \\
n / 2
\end{array}\right)\right\} d x=M(n) .
\end{aligned}
$$

В случае нечетного $n$ положим

$$
M_{3}^{\prime}(n)=\bigcup_{|t| \leqslant n 2^{n / 2}} M_{3}\left(n, z_{1}+t\right) .
$$

Пользуясь теоремой 1.4, аналогично убеждаемся, что

$$
M_{3}^{\prime}(n) \sim M(n)
$$

Из (1.2) и (1.3) следует, что почти все функции из $M(n)$ принадлежат множеству $M_{0}^{\prime}(n)$ при четном $n$ и множеству $M_{3}^{\prime}(n)$ при нечетном $n$.

\section{§ 1.4. Замкнутые классы монотонных булевых функций}

Пусть $A$-некоторое множество булевых функций. Замыканием множества $A$ называется совокупность всех булевых функций, представимых в виде формул через функции множества $A$. Замыкание множества $A$ обозначается через $[A]$.

Класс $A$ булевых функций назьвается замкнутым, если $A=[A]$.

Э. Пост [226] описал все замкнутые классы булевых функций. Описание этих классов содержится также в монографиях [190], [338]. Мы будем использовать терминологию и обозначения из [338].

Среди замкнутых классов булевых функций имеется счетное число классов, состоящих из монотонных функций. В частности, одним из таких классов является класс всех монотонных булевых функций.

Теперь перечислим все замкнутые классы монотонных булевых функций. Предварительно напомним определения используемых понятий.

Говорят, что булева функция $f\left(x_{1}, \ldots, x_{n}\right)$ сохраняет константу 0 (константу 1 ), если $f(0, \ldots, 0)=0(f(1, \ldots, 1)=1)$.

Говорят, что булева функция $f\left(x_{1}, \ldots, x_{n}\right)$ сохраняет константы 0 и 1 , если $f(0, \ldots, 0)=0$ и $f(1, \ldots, 1)=1$.

Булева функция $f\left(x_{1}, \ldots, x_{n}\right)$ назьвается самодвойственной, если $f\left(x_{1}, \ldots, x_{n}\right)=$ $\bar{f}\left(\bar{x}_{1}, \ldots, \bar{x}_{n}\right)$, т.е. на любых двух противоположных наборах функция $f$ принимает различные значения.

Замкнутыми классами монотонных булевых функций являются следующие: 
$A_{1}$ - множество всех монотонных булевых функций;

$A_{2}$ - множество всех монотонных булевых функций, сохраняюших константу 1 ;

$A_{3}$ - множество всех монотонных булевых функций, сохраняющих константу 0;

$A_{4}$ - множество всех монотонных булевых функций, сохраняюших константы 0 и 1 ;

$F_{2}^{m}$ - множество таких монотонных булевых функций $f$, сохраняющих константы

0 и 1 , что у любых $m$ наборов, на которых $f$ равна 0 , имеется по крайней мере одна общая нулевая компонента, $m=2,3, \ldots$;

$F_{3}^{m}$ - множество таких монотонных булевых функций $f$, что у любых $m$ наборов, на которых $f$ равна 0 , имеется по крайней мере одна обшая нулевая компонента, $m=$ $2,3, \ldots$;

$F_{6}^{m}$ - множество таких монотонных булевых функций $f$, сохраняющих константы 0 и 1 , что у любых $m$ наборов, на которых $f$ равна 1 , имеется по крайней мере одна обшая единичная компонента, $m=2,3, \ldots$;

$F_{7}^{m}$ - множество таких монотонных булевых функций $f$, что у любых $m$ наборов, на которых $f$ равна 1 , имеется по крайней мере одна общая единичная компонента, $m=2,3, \ldots$;

$F_{2}^{\infty}$ - множество таких монотонных булевых функций $f$, сохраняющих константы 0 и 1 , что у всех наборов, на которых $f$ равна 0 , имеется по крайней мере одна общая нулевая компонента;

$F_{3}^{\infty}$-множество монотонных булевых функций $f$ таких, что все наборы, на которых $f$ равна 0, имеют по крайней мере одну обшую нулевую компоненту;

$F_{6}^{\infty}$ - множество таких монотонных булевых функций $f$, сохраняющих константы 0 и 1 , что у всех наборов, на которых $f$ равна 1 , имеется по крайней мере одна общая единичная компонента;

$F_{7}^{\infty}$-множество монотонных булевых функций $f$ таких, что все наборы, на которых $f$ равна 1 , имеют по крайней мере одну общую единичную компоненту;

$D_{2}$ - множество самодвойственных монотонных булевых функций.

Для произвольного множества булевых функций $H$ через $H(n)$ обозначается совокупность функций из $H$ от переменных $x_{1}, \ldots, x_{n}$.

Так как $A_{1}(n)=M(n)$, то для величины $\left|A_{i}(n)\right|, 1 \leqslant i \leqslant 4$, справедливы асимптотики, указанные в теореме 1.1. Легко также видеть, что при любом $m \geqslant 2$ и $n \rightarrow \infty$

$$
\left|F_{2}^{m}(n)\right| \sim\left|F_{3}^{m}(n)\right|, \quad\left|F_{6}^{m}(n)\right| \sim\left|F_{7}^{m}(n)\right|,
$$

а также

$$
\left|F_{2}^{\infty}(n)\right| \sim\left|F_{3}^{\infty}(n)\right|, \quad\left|F_{6}^{\infty}(n)\right| \sim\left|F_{7}^{\infty}(n)\right| .
$$

Убедимся в том, что при любом $n \geqslant 1$

$$
\left|F_{3}^{\infty}(n)\right|=\left|F_{7}^{\infty}(n)\right|
$$

и при любом $m \geqslant 2$

$$
\left|F_{3}^{m}(n)\right|=\left|F_{7}^{m}(n)\right| \text {. }
$$

С этой целью каждой булевой функции $f\left(x_{1}, \ldots, x_{n}\right)$ поставим в соответствие булеву функцию $g_{f}\left(x_{1}, \ldots, x_{n}\right)$ такую, что

$$
g_{f}(\widetilde{\alpha})= \begin{cases}0, & \text { если } f(\overline{\widetilde{\alpha}})=1 ; \\ 1, & \text { если } f(\overline{\widetilde{\alpha}})=0 .\end{cases}
$$


Ясно, что это соответствие является взаимно однозначным. Вместе с тем если $f$ - монотонная функция, то $g_{f}$ - монотонная функция. Это следует из того, что если набор $\widetilde{\alpha}=\left(\alpha_{1}, \ldots, \alpha_{n}\right)$ является нижней единицей монотонной булевой функции $f\left(x_{1}, \ldots, x_{n}\right)$, то набор $\overline{\widetilde{\alpha}}=\left(\bar{\alpha}_{1}, \ldots, \bar{\alpha}_{n}\right)$ является верхним нулем функции $g_{f}\left(x_{1}, \ldots, x_{n}\right)$.

Из этого факта следует, что функция $f$ принадлежит множеству $F_{3}^{\infty}(n)$ (или $\left.F_{3}^{m}(n)\right)$ тогда и только тогда, когда $g_{f}$ принадлежит множеству $F_{7}^{\infty}(n)$ (или $F_{7}^{m}(n)$ ). Следовательно, при любом $n \geqslant 1$

$$
\left|F_{3}^{\infty}(n)\right|=\left|F_{7}^{\infty}(n)\right|
$$

и при любом $n \geqslant 1, m=2,3, \ldots$,

$$
\left|F_{3}^{m}(n)\right|=\left|F_{7}^{m}(n)\right| .
$$

Поэтому достаточно рассмотреть только множества $D_{2}(n), F_{7}^{\infty}(n)$ и $F_{7}^{m}(n), m=$ $2,3, \ldots$. Для таких множеств справедливы следующие утверждения.

Teорема 1.5 [158] (см. также [159] и [160]). При нечетном $n \rightarrow \infty$

$$
\left|F_{7}^{\infty}(n)\right| \sim n \cdot 2^{\left(\begin{array}{c}
n-1 \\
(n-1) / 2
\end{array}\right)} \exp \left\{\left(\begin{array}{c}
n-1 \\
(n-3) / 2
\end{array}\right)\left(2^{-(n-1) / 2}+n^{2} 2^{-n-4}-n 2^{-n-2}\right)\right\},
$$

а при четном $n \rightarrow \infty$

$$
\begin{aligned}
&\left|F_{7}^{\infty}(n)\right| \sim n \cdot 2^{\left(\begin{array}{c}
n-1 \\
n / 2
\end{array}\right)+1} \exp \left\{\left(\begin{array}{c}
n-1 \\
n / 2+1
\end{array}\right)\left(2^{-n / 2-1}-n^{2} 2^{-n-5}-3 n 2^{-n-4}\right)\right. \\
&\left.+\left(\begin{array}{c}
n-1 \\
n / 2-1
\end{array}\right)\left(2^{-n / 2}+n^{2} 2^{-n-3}-n 2^{-n-2}\right)\right\} .
\end{aligned}
$$

Справедливость теоремы 1.5 легко устанавливается с использованием теоремы 1.1, метода включения и исключения по числу обших единичных компонент в наборах из $E^{n}$, на которых функции из $F_{7}^{\infty}(n)$ равны 1, и неравенств Бонферрони [64].

Теорема 1.6. При нечетном $n \rightarrow \infty$

$$
\begin{gathered}
\left|F_{7}^{2}(n)\right| \sim 2^{\left(\begin{array}{c}
n \\
(n+1) / 2
\end{array}\right)} \exp \left\{\left(\begin{array}{c}
n \\
(n-1) / 2
\end{array}\right)\left(2^{-(n+3) / 2}+n 2^{-n-5}\right)\right. \\
\left.+\left(\begin{array}{c}
n \\
(n+3) / 2
\end{array}\right)\left(2^{-(n+3) / 2}+n^{2} 2^{-n-6}\right)\right\},
\end{gathered}
$$

а при четном $n \rightarrow \infty$

$$
\begin{gathered}
\left|F_{7}^{2}(n)\right| \sim 2^{\left(\begin{array}{c}
n \\
n / 2+1
\end{array}\right)} \exp \left\{\left(\begin{array}{c}
n \\
n / 2
\end{array}\right)\left(2^{-n / 2}+3 n^{2} 2^{-n-5}+n 2^{-n-4}\right)\right. \\
\left.+\left(\begin{array}{c}
n \\
n / 2+2
\end{array}\right)\left(2^{-n / 2-2}+n^{2} 2^{-n-7}\right)\right\} .
\end{gathered}
$$

Асимптотическая формула (1.6) и нижняя оценка для $\left|F_{7}^{2}(n)\right|$ при четных $n$, совпадающая с правой частью из (1.7), имеются в [156], [157], [159], [160] и доказаны в [158]. Асимптотическое равенство (1.7) установил А. А. Сапоженко в [262]. 
ТЕОРемА 1.7. При любом фиксированном $m \geqslant 3 u n \rightarrow \infty$

$$
\left|F_{7}^{m}(n)\right| \sim\left|F_{7}^{\infty}(n)\right|
$$

т.е. при нечетном $n \rightarrow \infty$ u $m \geqslant 3$

$$
\left|F_{7}^{m}(n)\right| \sim n 2^{\left(\begin{array}{c}
n-1 \\
(n-1) / 2
\end{array}\right)} \exp \left\{\left(\begin{array}{c}
n-1 \\
(n-3) / 2
\end{array}\right)\left(2^{-(n-1) / 2}+n^{2} 2^{-n-4}-n 2^{-n-2}\right)\right\},
$$

а при четном $n \rightarrow \infty u m \geqslant 3$

$$
\begin{gathered}
\left|F_{7}^{m}(n)\right| \sim n 2^{\left(\begin{array}{c}
n-1 \\
n / 2
\end{array}\right)+1} \exp \left\{\left(\begin{array}{c}
n-1 \\
n / 2+1
\end{array}\right)\left(2^{-n / 2-1}-n^{2} 2^{-n-5}-3 n 2^{-n-4}\right)\right. \\
\left.+\left(\begin{array}{c}
n-1 \\
n / 2-1
\end{array}\right)\left(2^{-n / 2}+n^{2} 2^{-n-3}-n 2^{-n-2}\right)\right\} .
\end{gathered}
$$

Этот результат анонсирован в [156], [158]. Полное доказательство этого факта пока не опубликовано.

ТЕорема 1.8. При нечетном $n \rightarrow \infty$

$$
\begin{gathered}
\left|D_{2}(n)\right| \sim 2^{\left(\begin{array}{c}
n-1 \\
(n+1) / 2
\end{array}\right)} \exp \left\{\left(\begin{array}{c}
n-1 \\
(n-1) / 2
\end{array}\right)\left(2^{-(n-1) / 2}+3 n^{2} 2^{-n-4}-n 2^{-n-3}\right)\right. \\
\left.+\left(\begin{array}{c}
n-1 \\
(n+3) / 2
\end{array}\right)\left(2^{-(n+3) / 2}+n^{2} 2^{-n-6}-n 2^{-n-5}\right)\right\}
\end{gathered}
$$

a при четном $n \rightarrow \infty$

$$
\begin{aligned}
\left|D_{2}(n)\right| \sim 2^{\left(\begin{array}{c}
n-1 \\
n / 2
\end{array}\right)} \exp \left\{\left(\begin{array}{c}
n-1 \\
n / 2-1
\end{array}\right)\left(2^{-n / 2-1}+n 2^{-n-4}\right)\right. \\
\left.\quad+\left(\begin{array}{c}
n-1 \\
n / 2+1
\end{array}\right)\left(2^{-n / 2-1}+n^{2} 2^{-n-5}-n 2^{-n-4}\right)\right\} .
\end{aligned}
$$

Утверждение теоремы 1.8 легко следуют из теоремы 1.6 и равенства

$$
\left|D_{2}(n)\right|=\left|F_{7}^{2}(n-1)\right|
$$

которое обнаружил А. А. Сапоженко [262].

\section{$\S$ 1.5. Структура типичных функций из замкнутых классов}

Опишем структуру типичных функций из $F_{7}^{\infty}(n)$ и $D_{2}(n)$. Начнем с множества $F_{7}^{\infty}(n)$. Во-первых, почти каждая функция $f \in F_{7}^{\infty}(n)$ обладает следуюшим свойством: во всех наборах, на которых $f$ равна 1 , имеется только одна общая единичная компонента. Далее, обозначим через $F_{7, i}^{\infty}(n)$ множество таких функций $f \in F_{7}^{\infty}(n)$, что во всех наборах, на которых $f$ равна 1 , только $i$-я компонента является единичной.

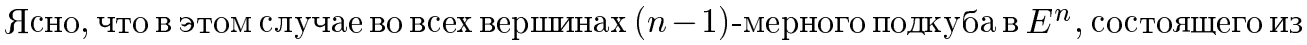
таких вершин $\left(\alpha_{1}, \ldots, \alpha_{n}\right)$, что $\alpha_{i}=0$, каждая функция $f$ из $F_{7, i}^{\infty}(n)$ равна 0, а во всех 
вершинах параллельного $(n-1)$-мерного подкуба функция $f$ может быть произвольной монотонной булевой функцией. Следовательно, структура типичных функций из $F_{7, i}^{\infty}(n)$ в этом подкубе совпадает со структурой типичных функций из $M(n-1)$.

Приведем некоторые сведения о структуре типичных функций из $F_{7}^{2}(n)$. В случае четного $n$ типичная функция $f \in F_{7}^{2}(n)$ является такой, что

1 ) все нижние единищы функции $f$ находятся в $E^{n, n / 2}, E^{n, n / 2+1}$ и $E^{n, n / 2+2}$;

2) функция $f$ равна 1 во всех вершинах из $E^{n, n / 2+3}, \ldots, E^{n, n}$;

$3)$ в $E^{n, n / 2}\left(E^{n, n / 2+2}\right)$ функция $f$ имеет не более $2^{n / 2}$ нижних единиц, множество этих единиц в каждом таком слое состоит из одноэлементных и двухэлементных связок и число двухэлементных связок не превосходит $n^{4}$;

4) в $E^{n, n / 2+1}$ функция $f$ имеет не менее $\frac{1}{2}\left(\begin{array}{c}n \\ n / 2+1\end{array}\right)-n 2^{n / 2}$ и не более $\frac{1}{2}\left(\begin{array}{c}n \\ n / 2+1\end{array}\right)+$ $n 2^{n / 2}$ нижних единиц.

В случае нечетного $n$ типичная функция $f \in F_{7}^{2}(n)$ является такой, что

1 ) все нижние единицы функции $f$ находятся в $E^{n,(n-1) / 2}, E^{n,(n+1) / 2}$ и $E^{n,(n+3) / 2}$;

2) функшия $f$ равна 1 во всех вершинах из $E^{n,(n+5) / 2}, \ldots, E^{n, n}$;

$3)$ в $E^{n,(n-1) / 2}\left(E^{n,(n+3) / 2}\right)$ функция $f$ имеет не более $2^{n / 2}$ нижних единиц, множество этих единиц в каждом таком слое состоит из одноэлементных и двухэлементных связок и число двухэлементных связок не превосходит $n^{4}$;

4) в $E^{n,(n+1) / 2}$ функшия $f$ имеет не менее $\frac{1}{2}\left(\begin{array}{c}n \\ (n+1) / 2\end{array}\right)-n 2^{n / 2}$ и не более $\frac{1}{2}\left(\begin{array}{c}n \\ (n+1) / 2\end{array}\right)+$ $n 2^{n / 2}$ нижних единиц.

\section{$\S$ 1.6. Еще одно множество монотонных булевых функций}

Рассматриваемые ниже множества функций из $M(n)$ возникли в связи с изучением статистических свойств одного класса нелинейных фильтров - стековых фильтров (см. § 3.5). По-видимому, эти множества представляют более общий интерес.

При четных и нечетных $n$ множества функций определяются по-разному. Начнем со случая четных $n$.

При четном $n$ через $M^{0}(n, r, z, v)$ обозначается множество функций из $M(n)$ таких, что

$1)$ все нижние единицы функции $f$ находятся в $E^{n, n / 2-1}, E^{n, n / 2}$ и $E^{n, n / 2+1}$;

2) функция $f$ равна 1 во всех вершинах из $E^{n, n / 2+2}, \ldots, E^{n, n}$;

3 ) функция $f$ имеет $r$ нижних единищ в $E^{n, n / 2-1}$ и множество этих единиц состоит из одноэлементных и двухэлементных связок, а число двухэлементных связок не превосходит $n^{4}$;

4 ) функция $f$ имеет $v$ верхних нулей в $E^{n, n / 2+1}$ и множество этих нулей состоит из одноэлементных и двухэлементных связок, а число двухэлементных связок не превосходит $n^{4}$;

$5)$ в $E^{n, n / 2}$ имеется $z$ вершин, в которых $f$ равна 1 .

Tеорема 1.9 [162]. Пусть п четно,

$$
r_{0}=r_{0}(n)=v_{0}=v_{0}(n)=\left\lfloor\left(\begin{array}{c}
n \\
n / 2-1
\end{array}\right) 2^{-n / 2-1}\right\rfloor, \quad z_{0}=\frac{1}{2}\left\lfloor\left(\begin{array}{c}
n \\
n / 2
\end{array}\right)\right\rfloor .
$$


Тогда при любьх $k, u, t$ таких, что $|k| \leqslant n 2^{n / 4},|u| \leqslant n 2^{n / 2},|t| \leqslant n 2^{n / 4}, u n \rightarrow \infty$ справедливо соотношение

$$
\begin{aligned}
&\left|M^{0}\left(n, r_{0}+k, z_{0}+u, v_{0}+t\right)\right| \sim \sqrt{2^{n+1} /\left(\pi^{3}\left(\begin{array}{c}
n \\
n / 2
\end{array}\right)\right)^{3}}|M(n)| \\
& \times \exp \left\{-\frac{2^{n / 2}}{\left(\begin{array}{c}
n \\
n / 2-1
\end{array}\right)}\left(k^{2}+t^{2}\right)-\frac{2 u^{2}}{\left(\begin{array}{c}
n \\
n / 2
\end{array}\right)}\right\} .
\end{aligned}
$$

При нечетных $n$ рассматриваются два подмножества $M^{1}(n, r, z, v)$ и $M^{2}(n, r, z, v)$. Множество $M^{1}(n, r, z, v)$ состоит из функций $f \in M(n)$ таких, что

$1)$ все нижние единицы функщии $f$ находятся в $E^{n,(n-3) / 2}, E^{n,(n-1) / 2}$ и $E^{n,(n+1) / 2}$;

2) функция $f$ равна 1 во всех вершинах из $E^{n,(n+3) / 2}, \ldots, E^{n, n}$;

$3)$ функция $f$ имеет $r$ нижних единиц в $E^{n,(n-3) / 2}$, множество этих единиц состоит из одноэлементных и двухэлементных связок, а число двухэлементных связок не превосходит $n^{4}$;

4 ) функция $f$ имеет $v$ верхних нулей в $E^{n,(n+1) / 2}$, множество этих нулей состоит из одноэлементных и двухэлементных связок, а число двухэлементных связок не превосходит $n^{4}$;

$5)$ в $E^{n,(n-1) / 2}$ имеется $z$ вершин, в которых $f$ равна 1 .

Теорема 1.10 [162]. Пусть $n$ нечетно и

$$
\begin{gathered}
r_{1}=r_{1}(n)=\left\lfloor\left(\begin{array}{c}
n \\
(n-3) / 2
\end{array}\right) 2^{-(n+3) / 2}\right\rfloor, \\
v_{1}=v_{1}(n)=\left\lfloor\left(\begin{array}{c}
n \\
(n+1) / 2
\end{array}\right) 2^{-(n+1) / 2}\right\rfloor, \\
z_{1}=z_{1}(n)=\left\lfloor\frac{1}{2}\left(\left(\begin{array}{c}
n \\
(n+1) / 2
\end{array}\right)+r_{1}(n+3) / 2-v_{1}(n+1) / 2\right)\right\rfloor .
\end{gathered}
$$

Тогда при любьх $k, u, t$ таких, что $|k| \leqslant n 2^{n / 4},|u| \leqslant n 2^{n / 2},|t| \leqslant n 2^{n / 4}$, u $n \rightarrow \infty$ справедливо соотношение

$$
\begin{aligned}
& \left|M^{1}\left(n, r_{1}+k, z_{1}+u, v_{1}+t\right)\right| \sim \frac{1}{2} \sqrt{2^{n+1} /\left(\pi^{3}\left(\begin{array}{c}
n \\
(n-1) / 2
\end{array}\right)^{3}\right)}|M(n)| \\
& \quad \times \exp \left\{-\frac{2^{(n+1) / 2}}{\left(\begin{array}{c}
n \\
(n-3) / 2
\end{array}\right)} k^{2}-\frac{2^{(n-1) / 2}}{\left(\begin{array}{c}
n \\
(n+1) / 2
\end{array}\right)} t^{2}-\frac{2 u^{2}}{\left(\begin{array}{c}
n \\
(n-1) / 2
\end{array}\right)}\right\} .
\end{aligned}
$$

Наконец, множество $M^{2}(n, r, z, v)$ состоит из функций $f \in M(n)$ таких, что

1) все нижние единицы функции $f$ находятся в $E^{n,(n-1) / 2}, E^{n,(n+1) / 2}$ и $E^{n,(n+3) / 2}$;

2) функция $f$ равна 1 во всех вершинах из $E^{n,(n+5) / 2}, \ldots, E^{n, n}$;

3 ) функция $f$ имеет $r$ нижних единиц в $E^{n,(n-1) / 2}$, множество этих единиц состоит из одноэлементных и двухэлементных связок, а число двухэлементных связок не превосходит $n^{4}$;

4 ) функция $f$ имеет $v$ верхних нулей в $E^{n,(n+3) / 2}$, множество этих нулей состоит из одноэлементных и двухэлементных связок, а число двухэлементных связок не превосходит $n^{4}$;

$5)$ в $E^{n,(n+1) / 2}$ имеется $z$ вершин, в которых $f$ равна 1 . 
TЕОРема 1.11 [162]. Пусть $n$ нечетно и

$$
\begin{gathered}
r_{2}=r_{2}(n)=\left\lfloor\left(\begin{array}{c}
n \\
(n-1) / 2
\end{array}\right) 2^{-(n+1) / 2}\right\rfloor, \\
v_{2}=v_{2}(n)=\left\lfloor\left(\begin{array}{c}
n \\
(n+3) / 2
\end{array}\right) 2^{-(n+3) / 2}\right\rfloor, \\
z_{2}=z_{2}(n)=\left\lfloor\frac{1}{2}\left(\left(\begin{array}{c}
n \\
(n+1) / 2
\end{array}\right)+r_{2}(n+1) / 2-v_{2}(n+3) / 2\right)\right\rfloor .
\end{gathered}
$$

Тогда при любьх $k, u, t$ таких, что $|k| \leqslant n 2^{n / 4},|u| \leqslant n 2^{n / 2},|t| \leqslant n 2^{n / 4}$, u $n \rightarrow \infty$ справедливо соотношение

$$
\begin{aligned}
& \left|M^{2}\left(n, r_{2}+k, z_{2}+t, v_{2}+t\right)\right| \sim \frac{1}{2} \sqrt{2^{n+1} /\left(\pi^{3}\left(\begin{array}{c}
n \\
(n-1) / 2
\end{array}\right)^{3}\right)}|M(n)| \\
& \quad \times \exp \left\{-\frac{2^{(n-1) / 2}}{\left(\begin{array}{c}
n \\
(n-1) / 2
\end{array}\right)} k^{2}-\frac{2^{(n+1) / 2}}{\left(\begin{array}{c}
n \\
(n+3) / 2
\end{array}\right)} t^{2}-\frac{2 u^{2}}{\left(\begin{array}{c}
n \\
(n+1) / 2
\end{array}\right)}\right\} .
\end{aligned}
$$

Пусть при $i=0,1,2$

$$
M^{i}(n)=\bigcup_{|k| \leqslant n 2^{n / 4}} \bigcup_{|u| \leqslant n 2^{n / 2}} \bigcup_{|t| \leqslant n 2^{n / 4}} M^{i}\left(n, r_{i}+k, z_{i}+u, v_{i}+t\right)
$$

Тогда согласно теореме 1.9 имеем

$$
\begin{aligned}
\left|M^{0}(n)\right| & \sim \sqrt{2^{n+1} /\left(\pi^{3}\left(\begin{array}{c}
n \\
n / 2
\end{array}\right)^{3}\right)}|M(n)| \\
& \times \sum_{|k| \leqslant n 2^{n / 4}|u| \leqslant n 2^{n / 2}|t| \leqslant n 2^{n / 4}} \exp \left\{-\frac{2^{n / 2}}{\left(\begin{array}{c}
n \\
n / 2-1
\end{array}\right)}\left(k^{2}+t^{2}\right)-\frac{2 u^{2}}{\left(\begin{array}{c}
n \\
n / 2
\end{array}\right)}\right\} \\
& \sqrt{2^{n+1} /\left(\pi^{3}\left(\begin{array}{c}
n \\
n / 2
\end{array}\right)^{3}\right)}|M(n)| \int_{-\infty}^{\infty} \exp \left\{-\frac{2^{n / 2} x^{2}}{\left(\begin{array}{c}
n \\
n / 2-1
\end{array}\right)}\right\} d x \\
& \times \int_{-\infty}^{\infty} \exp \left\{-\frac{2^{n / 2} y^{2}}{\left(\begin{array}{c}
n \\
n / 2-1
\end{array}\right)}\right\} d y \int_{-\infty}^{\infty} \exp \left\{-\frac{2 z^{2}}{\left(\begin{array}{c}
n \\
n / 2
\end{array}\right)}\right\} d z=|M(n)| .
\end{aligned}
$$

Пользуясь теоремами 1.10 и 1.11 , аналогично убеждаемся в том, что

$$
\left|M^{1}(n)\right| \sim \frac{1}{2}|M(n)|, \quad\left|M^{2}(n)\right| \sim \frac{1}{2}|M(n)| .
$$

Из (1.8) и (1.9) следует, что почти все функции из $M(n)$ принадлежат множеству $M^{0}(n)$ при четном $n$ и множеству $M^{1}(n) \cup M^{2}(n)$ при нечетном $n$. 


\section{§ 1.7. Монотонные булевы функции и шпернеровы семейства}

Пусть $S$ - произвольное конечное множество. Семейство $\mathscr{F}$ подмножеств множества $S$ назьвается шпернеровыл, если ни один член семейства $\mathscr{F}$ не содержится в другом члене семейства $\mathscr{F}$.

Шпернеровы семейства изучаются давно. Еще в 1927 г. Э. Шпернер [290] доказал следующее утверждение.

ТЕОРЕМа 1.12. В максимальном шпернеровом семействе п-элементного множества содержится $\left(\begin{array}{c}n \\ \lfloor n / 2\rfloor\end{array}\right)$ әлементов. В случае четного $n$ максимальное шпернерово семейство единственно и состоит из всех $n / 2$-элементных подмножеств $n$-элементного множсества. $B$ случае нечетного $n$ имеются два максимальных шпернеровых семейства: одно семейство состоит из всех $(n-1) / 2$-элементных подмноэсеств, а другое - из всех $(n+1) / 2$-элементных подмножеств.

Эта теорема многократно переоткрьвалась и передоказывалась (см., например, [178], [198]).

В течение многих лет исследуются различные задачи, относящиеся к шпернеровьм семействам (см., например, [61]-[63], [138]).

Мы рассмотрим только те из них, которые тесно связаны с монотонными булевыми функциями. Между монотонными булевыми функциями от $n$ переменных и шшернеровьми семействами $n$-элементного множества $S$ имеется взаимно однозначное соответствие. Пусть $\mathscr{F}=\left\{S_{1}, \ldots, S_{k}\right\}$ - произвольное шпернерово семейство $n$-элементного множества $S$. Каждому $S_{i}, 1 \leqslant i \leqslant k$, ставится в соответствие набор $\widetilde{\alpha}_{i}=\left(\alpha_{i}^{1}, \ldots, \alpha_{i}^{n}\right)$ из $E^{n}$ такой, что

$$
\alpha_{i}^{j}= \begin{cases}1, & \text { если } j \text {-й элемент из } S \text { принадлежит множеству } S_{i}, \\ 0 & \text { в противном случае. }\end{cases}
$$

Такой набор $\widetilde{\alpha}_{i}$ обьчно назьвается характеристическим набором множества $S_{i}$. Ясно, что все характеристические наборы семейства $\mathscr{F}$ - попарно несравнимы. Поэтому эти наборы можно взять в качестве нижних единиц функции из $M(n)$. Наоборот, если наборы $\widetilde{\alpha}_{1}, \ldots, \widetilde{\alpha}_{k}$ из $E^{n}$ - нижние единицы функции из $M(n)$, то они являются характеристическими только одного шшернерова семейства $n$-элементного множества.

Из этого соответствия следует, что число функщий из $M(n)$ равно числу шшернеровых семейств $n$-элементного множества, а асимптотики для числа функций из $M(n)$, приведенные в теореме 1.1 , суть асимптотики для числа шпернеровых семейств $n$-элементного множества.

Семейство $\mathscr{F}$ подмножеств заданного множества называется $m$-неразделеннылм, если любые $m$ элементов семейства $\mathscr{F}$ имеют непустое пересечение.

Семейство $\mathscr{F}$ подмножеств множества $S$ называется сильно неразделенным, если по крайней мере один элемент из $S$ принадлежит всем элементам семейства $\mathscr{F}$.

Ясно, что число функций из $F_{7}^{m}(n)$ равно числу $m$-неразделенных шпернеровых семейств $n$-элементного множества, а асимптотики для числа функций из $F_{7}^{m}(n)$, приведенные в теоремах 1.6 и 1.7 , суть асимптотики для числа $m$-неразделенных шпернеровых семейств $n$-элементного множества. 
Аналогично число функций из $F_{7}^{\infty}(n)$ равно числу сильно неразделенных шшернеровых семейств $n$-элементного множества, а асимптотики для числа функций из $F_{7}^{\infty}(n)$, приведенные в теореме 1.5 , суть асимптотики для числа сильно неразделенных шпернеровых семейств $n$-элементного множества.

\section{§ 1.8. Развитие метода. Другие результаты}

Метод доказательства теоремы 1.1, описанньй в [152], оказался весьма общим и использовался автором при доказательстве теорем 1.3-1.11. Конечно, при этом пришлось учитывать специфические свойства функций из рассматриваемых классов.

Метод оказался применим также для решения других задач дискретной математики, имеющих с проблемой Дедекинда близкую комбинаторную природу. Эта близость состоит в том, что при решении каждой такой задачи возникает потребность в нахождении числа подмножеств исходного множества, которые имеют границу заданного размера.

Например, к таким задачам относится задача о нахождении числа всех двоичных кодов заданной длины с расстоянием 2 (подмножество вершин $A$ из $E^{n}$ назьвается двоичным кодом длины $n$ с расстоянием 2 , если расстояние Хемминга между любьми двумя наборами из $A$ не меньше 2). В работе [161] (см. также [154] и [155]) доказано, что при $n \rightarrow \infty$ число таких кодов асимптотически равно $2 \sqrt{e} 2^{2^{n-1}}$.

Метод из [152] получил дальнейшее обобщение и развитие в работах А. А. Сапоженко [259]-[262], [264], [265], [267] (см. также [266]).

Основное обобщение заключается в следующем. При нахождении асимптотик для числа функций из $M(n)$ главная трудность заключается в нахождении достаточно точных верхних оценок для числа таких множеств вершин, которые расположены в одном слое булева куба $E^{n}$ и имеют 1-тень заданного размера. При этом фактически рассматривались двудольные графы, в каждом из которых все вершины одной доли имеют одинаковую степень, а каждая вершина одной доли смежна с конкретными вершинами другой доли.

Вместо таких двудольных графов А.А. Сапоженко рассматривал произвольные двудольные графы, произвольные графы и некоторые другие классы графов. В таких более общих ситуациях при решении задач возникают дополнительные трудности. В этих случаях вместо 1-тени естественно использовать понятие границы, определяемое следующим образом.

Под границей множества вершин $A$ в произвольном графе $G$ понимается множество тех вершин в $G$, каждая из которых смежна хотя бы с одной вершиной из $A$ и не принадлежит $A$.

Основные результаты А. А. Сапоженко состоят в следующем. В работе [259] он рассматривал двудольные $n$-вершинные графы при определенных ограничениях на степени вершин. Главным результатом этой работы является верхняя оценка для числа связных подмножеств вершин с границей фиксированного размера (подмножество $A$ с границей $\Gamma(A)$ в графе $G$ назьвается связным, если подграф с множеством вершин $A \cup \Gamma(A)$ графа $G$ является связным).

В [264] (см. также [265]) А. А. Сапоженко получил аналогичные оценки в случае произвольных графоов.

В работе [261] он получил асимптотику для числа антицепей (шпернеровых 
семейств) в частично упорядоченных $n$-Элементных множествах, диаграммы которых являются двудольными графами с некоторыми ограничениями.

Продолжением этой работы является работа [262]. В ней рассмотрена задача о числе антицепей в $m$-слойных частично упорядоченных $n$-элементных множествах, т.е. множествах, которые задаются графами, в каждом из которых совокупность всех вершин разбита на $m$ упорядоченных слоев, $m \geqslant 2$, а смежными могут быть только вершины из соседних слоев. В [262] найдена асимптотика для числа антищепей в таких частично упорядоченных множествах, удовлетворяюших некоторым дополнительным ограничениям.

В работе [260] найдена асимптотика для числа монотонных функций, которые принимают значения 0 и 1 и заданы на двухслойных частично упорядоченных $n$-элементных множествах, удовлетворяющих специальньм ограничениям.

В статье [267] приводится другое доказательство асимптотик из [152] для числа функций из $M(n)$ и даются более простые доказательства результатов из работ [259], [261] и [262]. Обзор некоторых изложенных результатов имеется также в [266].

Информация из [152] о структуре типичных функций из $M(n)$ оказалась полезной при решении ряда задач дискретной математики.

Н. Н. Нурмеев [213] воспользовался этой информацией при исследовании сложности вычисления функций из $M(n)$ контактными схемами (см. $\S 2.3)$. Эта же информация использовалась в [34] и [35] при изучении сложности вычисления монотонных булевых функщий на параллельных вычислительных машинах с распределенной памятью (PRAMs и WRAMs).

В. А. Варданян [312] использовал информацию о структуре типичных функций из $M(n)$ при решении задачи о нахождении минимальных единичных динамических тестов для типичных функций из $M(n)($ см. $\S 3.4)$.

\section{Глава 2.}

\section{Сложность вычисления (реализации) монотонных булевых функций}

\section{§.1. Постановка задачи}

Построение (синтез) экономных схем таких, как булевы схемы (иначе: схемы из функциональных элементов), контактные схемы и т. д., является одной из важнейших проблем современной науки о вычислениях. Впервые достаточно подробно она рассматривалась К. Шенноном [275]. В обших чертах эта задача формулируется следуюшим образом. Заданы: запас элементарных средств (например, формулы элементарных функций, набор булевых функций и т. п.) и правила построения из них более сложных образований - схем. Предполагается, что каждая схема вычисляет (реализует) некоторую функцию. Для каждой функции из заданного класса требуется построить схему, которая выгисляет эту функцию.

Как правило, эта задача имеет много решений. Поэтому задача синтеза схем уточняется: требуется построить не какую-нибудь схему, вычисляющую данную функцию, а в том или ином смысле наилучшую (наиболее простую) схему. Обычно имеется тривиальный метод получения такой экстремальной схемы, основанный на переборе всех схем заранее ограниченной сложности. Однако такой метод мало эффективен, 
поскольку он требует перебора очень большого числа схем. Это основная причина изменения постановки задачи. Один из подходов состоит в исследовании универсальных методов синтеза, позволяющих строить почти минимальные схемы, выгисляюшие большую часть булевых функций из того или иного класса.

Название "универсальньй" связано с тем, что эти методы рассчитаны на построение схем, вычисляюших произвольные функции из рассматриваемого класса. Вместе с тем эти методы мало учитьвают специфику индивидуальных функций.

Задача о нахождении универсальных методов может быть сформулирована следующим образом.

Пусть $\mathscr{F}$ - некоторый класс булевых функций и $\Sigma$ - множество схем, которые можно использовать для вычисления функций из $\mathscr{F}$. Предполагается, что каждая функция из $\mathscr{F}$ может быть вычислена по крайней мере одной схемой из $\Sigma$. Пусть каждой схеме $S$ из $\Sigma$ поставлено в соответствие неотрицательное число $L(S)$, назьваемое $c л о ж c-$ ностью схемы $S$. Например, под сложностью контактной схемы обычно понимается число контактов в ней.

Через $L_{\Sigma}(f)$ обозначим нижнюю грань сложностей схем из $\Sigma$, вычисляюших булеву функщию $f$, и через $L_{\Sigma}^{\mathscr{F}}(n)$ - наибольшее из чисел $L_{\Sigma}\left(f\left(x_{1}, \ldots, x_{n}\right)\right)$, где максимум берется по всем функциям от $n$ переменных, принадлежащих множеству $\mathscr{F}$.

Требуется найти такой эффективный метод синтеза схем, которьй для любой функщии $f$ из $\mathscr{F}$, зависящей не более чем от $n$ переменных, позволяет строить такую схему $S$ из $\Sigma$, что $S$ вычисляет $f$ и имеет сложность, не превосходящую $L_{\Sigma}^{\mathscr{F}}(n)\left(1+\varepsilon_{n}\right)$, где $\varepsilon_{n} \rightarrow 0$ при $n \rightarrow \infty$.

Функция $L \underset{\Sigma}{\mathscr{F}}(n)$ впервые была введена К. Шенноном [276] при изучении сложности контактных схем, вычисляющих произвольные булевы функции. Впоследствии функции типа $L_{\Sigma}^{\mathscr{F}}(n)$ стали назьвать функциями Шеннона.

Одним из первых универсальных методов синтеза является метод Шеннона [276]. Этот метод не ориентирован на получение экономных контактных схем для последовательностей конкретных булевых функций. Однако его модификация, известная как метод каскадов, может давать весьма экономные контактные схемы и для индивидуальных функций.

Многие универсальные методы синтеза основаны на использовании метода локального кодирования, предложенного О.Б. Лупановым [180] (подробное изложение имеется в [183]). Ниже этот метод мы будем назьвать методом локального кодирования Лупанова.

Суть этого метода состоит в следуюшем. Все булевы функции из рассматриваемого класса кодируются последовательностями из нулей и единиц так, что по коду функция восстанавливается однозначно. Цель кодирования состоит в сжатии информации. Кодирование является локальным, т.е. для вычисления значения функции на произвольном наборе значений переменных достаточно знать только фрагмент кода не очень большой длины (в общем случае эти фрагменты могут иметь переменную длину). К тому же во многих случаях кодирование является "асимптотически наиболее экономным". Однако это условие не обязательно: если оно вьполняется, то почти все схемы получаются "асимптотически наилучшими” для достаточно мошных классов булевых функщий; если условие не вьполняется, схемы не будут “асимптотически наилучшими", но все же могут оказаться достаточно экономньми.

Булева схема, построенная с использованием этого метода и вычисляющая исход- 
ную функцию, состоит из нескольких блоков. Один блок по входному набору значений переменных выдает “координаты” фрагмента кода, другой блок по координатам фрагмента кода выдает сам фрагмент (этот блок обычно содержит большинство элементов схемы), и третий блок по фрагменту кода и входному набору значений переменных “просто" вычисляет значение функции. Описание этого метода имеется также в монографии [55; с. 124-148].

Поскольку вычисление функщии Шеннона, как правило, практически невозможно, ограничиваются исследованием ее асимптотического поведения.

Асимптотика функции Шеннона обьчно извлекается из асиптотически совпадающих нижней и верхней оценок для нее. При получении этих оценок используются разные соображения. Нижняя оценка получается следуюшим способом, обычно назьваемым мощностным методом.

Пусть $\mathscr{F}$ - рассматриваемьй класс булевых функций и $\mathscr{F}(n) \subset \mathscr{F}-$ множество функций от переменных $x_{1}, \ldots, x_{n}$. Обозначим через $N(n, k)$ верхнюю оценку (обычно достаточно грубую) для числа используемых схем (булевы схемы, контактные схемы и т.п.), которые имеют сложность не более $k=k(n)$ и вычисляют (реализуют) функции из $\mathscr{F}(n)$. Поскольку должно выполняться неравенство

$$
N(n, k)>|\mathscr{F}(n)|,
$$

функция Шеннона $L^{\mathscr{F}}(n)>k(n)$. Поэтому при установлении нижней оценки для функции Шеннона подбирают такое минимально возможное $k=k_{0}(n)$, чтобы вьполнялось неравенство (2.1).

Для получения верхней оценки предлагают метод синтеза схем, позволяющий для любой функщии из $\mathscr{F}(n)$ строить схему, сложность которой асимптотически не превосходит $k_{0}(n)$.

Поиск таких методов синтеза, как правило, весьма сложен.

\section{$\S$ 2.2. Сложность булевых схем}

В этом и последующих параграффах рассматриваются схемы, которые встречаются под названиями: схемы из функциональных элементов, комбинационные схемы, булевы схемы, схемы и т.п. В настояшей статье такие схемы мы будем называть булевыми схемами. Эти схемы можно определить следующим образом.

Пусть $x_{1}, x_{2}, \ldots$ - булевы переменные, $\mathscr{E}=\left\{f_{1}, \ldots, f_{r}\right\}$ - произвольное множество булевых функций, называемое базисом, и $n_{i}-$ число переменных функции $f_{i}, 1 \leqslant i \leqslant r$.

Пусть $S$ - произвольный конечный ориентированньй граф без ориентированных циклов, в котором некоторые вершины помечены символами из $\mathscr{E}$, а все дуги, направленные в одну вершину, перенумерованы. При этом предполагается, что вьполняются следуюшие требования:

1) отсутствуют дуги, направленные в вершины, которые помечены символами $x_{1}, x_{2}, \ldots$

2) число дуг, которые направлены в вершину с меткой $f_{i}$, равно $n_{i}, 1 \leqslant i \leqslant r$.

Такой граф $S$, в котором некоторые вершины помечены как выходные, назьвается булевой схемой в базисе $\mathscr{E}$.

Вершины схемы $S$, помеченные символами $x_{1}, x_{2}, \ldots$, назьваются ее входами, вершины с метками из $\mathscr{E}$ - элементами. Элементы реализуют булевы функции; при этом 
считается, что вершина с меткой $f_{i}$ является элементом, реализуюшим булеву функцию $f_{i}$ от $n_{i}$ переменных. Предполагается, что каждый элемент имеет только один выход, но если из некоторого элемента (вершины) исходит несколько дуг, то считается, что выход этого элемента подается на несколько входов других элементов. Все дуги схемы $S$ интерпретируются как каналы, по которьм происходит передача информации в направлении их ориентации. Входы схемы служат для ее подключения к источнику внешней информации, которая передается на входы элементов.

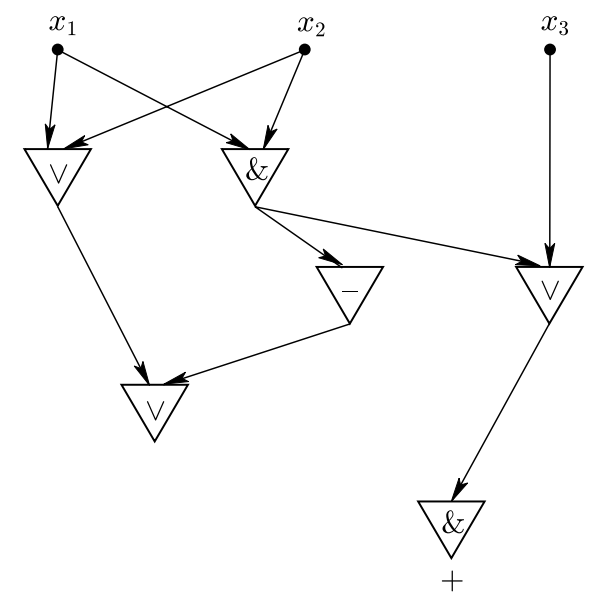

Нетрудно понять, что на выходе каждого элемента схемы $S$ вычисляется булева функция от тех переменных, которыми помечены входы схемы $S$.

Как обычно, полюсы (булевой) схемы изображают маленькими кружочками, а элементы - треугольниками. При таком изображении отпадает необходимость в указании номеров каналов, если только условиться в том, что все каналы, направленные к элементу, подключены к одной стороне треугольника и следуют друг за другом слева направо, а выходом элемента является противоположная вершина треугольника.

В качестве примера на рисунке приведена булева схема, состояшая из дизъюнкторов $(\vee)$, конъюнкторов $(\&)$ и инвертора $(-)$. На выходе этой схемы, помеченной символом “+”, вычисляется булева функция

$$
f\left(x_{1}, x_{2}, x_{3}\right)=\left(\left(x_{1} \vee x_{2}\right) \vee \overline{x_{1} \& x_{2}}\right) \&\left(\left(x_{1} \& x_{2}\right) \vee x_{3}\right)=x_{1} x_{2} \bar{x}_{3} \vee x_{3} .
$$

Теперь изложим результаты, относящиеся к сложности булевых схем, которые вычисляют (реализуют) функции из $M(n)$, т.е. монотонные булевы функции от $n$ переменных. При этом под сложностью булевой схемы понимается число элементов в ней, а функция Шеннона для таких схем в базисе $\mathscr{E}$ обозначается через $L_{\mathscr{E}}^{M}(n)$. Такая сложность схем часто назьвается размером схем.

Сначала приведем нижнюю оценку для $L_{\mathscr{E}}^{M}(n)$. Пусть $\mathscr{E}=\left\{f_{1}, \ldots, f_{r}\right\}$ и $s_{i}$ - число сушественных переменных булевой функции $f_{i}, 1 \leqslant i \leqslant r$. Введем величину $\rho(\mathscr{E})=$ $\min \frac{1}{s_{i}-1}$, где минимум берется по всем таким $i$, что $s_{i} \geqslant 2$.

Пользуясь неравенством

$$
\log _{2}|M(n)|>\left(\begin{array}{c}
n \\
\lfloor n / 2\rfloor
\end{array}\right)
$$


которое следует из (1.1), и леммой Д.3 из [183] (см. также [187; лемма 12]), легко убедиться в том, что при $n \rightarrow \infty$

$$
L_{\mathscr{E}}^{M}(n)>\frac{\rho(\mathscr{E})}{n}\left(\begin{array}{c}
n \\
\lfloor n / 2\rfloor
\end{array}\right)\left(1+\frac{\log _{2} n}{n}\right) .
$$

Основные трудности возникают при получении верхних оценок для функции Шеннона $L_{\mathscr{E}}^{M}(n)$ при разных $\mathscr{E}$.

В. И. Резник [254] получил следуюшую оценку:

$$
L_{\{\&, \vee,-\}}^{M}(n) \leqslant O\left(\left(\begin{array}{c}
n \\
\lfloor n / 2\rfloor
\end{array}\right) \frac{\log n}{n}\right),
$$

которая существенно отличается от нижней оценки (2.3).

Более точная верхняя оценка для $L_{\{\&, \vee,-\}}^{M}(n)$ была получена В.К. Коробковым в 1965 г. [146]. Используя метод локального кодирования Лупанова, он описал метод синтеза булевых схем в базисе $\{\&, \vee,-\}$, вычисляюших функции из $M(n)$, из которого извлекается следуюшая оценка:

$$
L_{\{\&, \vee,-\}}^{M}(n)<\frac{4.27}{n}\left(\begin{array}{c}
n \\
\lfloor n / 2\rfloor
\end{array}\right)\left(1+\varepsilon_{n}\right),
$$

где $\varepsilon_{n} \rightarrow 0$ при $n \rightarrow \infty$. Из (2.3) и (2.5) следует, что

$$
L_{\{\&, \vee,-\}}^{M}(n)=\Theta\left(\left(\begin{array}{c}
n \\
\lfloor n / 2\rfloor
\end{array}\right) / n\right) .
$$

Таким образом, В. К. Коробковым впервые был установлен порядок функции Шеннона $L_{\{\&, \vee,-\}}^{M}(n)$, а следовательно, порядок функции Шеннона $L_{\mathscr{E}}^{M}(n)$ при любом конечном полном базисе $\mathscr{E}$ в классе всех монотонных булевых функций.

Ж. Ансель [96], используя свой метод оценки числа функций из $M(n)$ и метод локального кодирования Лупанова, несколько улучшил оценку (2.5). Полученный им результат имеет вид

$$
L_{\{\&, \vee,-\}}^{M}(n)<\frac{\log _{2} 3}{n}\left(\begin{array}{c}
n \\
\lfloor n / 2\rfloor
\end{array}\right)\left(1+\varepsilon_{n}\right), \text { где } \varepsilon_{n} \rightarrow 0 \text { при } n \rightarrow \infty .
$$

Окончательное (с точностью до асимптотики) решение задачи о поведении функции $L_{\mathscr{E}}^{M}(n)$ было найдено А.Б. Угольниковым в 1976 г. [302]. Он описал такой метод синтеза булевых схем в произвольном конечном полном базисе $\mathscr{E}$, которьй для любой функции $f \in M(n)$ позволяет строить схему в базисе $\mathscr{E}$, которая вычисляет функцию $f$ и имеет не более $\frac{\rho(\mathscr{E})}{n}\left(\begin{array}{c}n \\ \lfloor n / 2\rfloor\end{array}\right)\left(1+\varepsilon_{n}\right)$ элементов, где $\varepsilon_{n} \rightarrow 0$ при $n \rightarrow \infty$. Следовательно, А.Б. Угольниковым доказано, что для любого конечного полного базиса $\mathscr{E}$

$$
L_{\mathscr{E}}^{M}(n) \leqslant \frac{\rho(\mathscr{E})}{n}\left(\begin{array}{c}
n \\
\lfloor n / 2\rfloor
\end{array}\right)\left(1+\varepsilon_{n}\right),
$$

где $\varepsilon_{n} \rightarrow 0$ при $n \rightarrow \infty$. Отсюда и из (2.3) следует

ТЕОРема 2.1. Пусть $\mathscr{E}-$ произвольный конечный полный базис для класса всех булевьх функиий. Тогда при $n \rightarrow \infty$

$$
L_{\mathscr{E}}^{M}(n) \sim \frac{\rho(\mathscr{E})}{n}\left(\begin{array}{c}
n \\
\lfloor n / 2\rfloor
\end{array}\right) .
$$

В том же году была опубликована статья Н. Пиппенджера [220] (см. также [221]), в которой установлен более точньй результат. В ней доказана следующая теорема. 
ТЕОРема 2.2. Пусть $\mathscr{E}-$ произвольныи й конечный полный базис для класса всех булевьх функиий. Тогда

$$
L_{\mathscr{E}}^{M}(n)-\frac{\rho(\mathscr{E})}{n}\left(\begin{array}{c}
n \\
\lfloor n / 2\rfloor
\end{array}\right) \leqslant O\left(\left(\begin{array}{c}
n \\
\lfloor n / 2\rfloor
\end{array}\right) \frac{\log n}{n^{2}}\right) .
$$

Доказательства теорем 2.1 и 2.2 основаны на использовании метода локального кодирования Лупанова и способа задания функций из $M(n)$, предложенного Д. Клейтманом и Дж. Марковским [139].

Н.П. Редькин исследовал сложность минимальных схем в базисе $\{\&, \vee\}$, вычисляющих функции из $M(n)$. В работе [251] он доказал, что

$$
L_{\{\&, \vee\}}^{M}(n) \leqslant O\left(\left(\begin{array}{c}
n \\
\lfloor n / 2\rfloor
\end{array}\right) / n\right) .
$$

Отсюда и из (2.3) следует, что

$$
L_{\{\&, \vee\}}^{M}(n)=\Theta\left(\left(\begin{array}{c}
n \\
\lfloor n / 2\rfloor
\end{array}\right) / n\right) .
$$

А.Е. Андреев исследовал более общую задачу - сложность булевых схем, вычисляюших функции из $M(n)$, в произвольных конечных полных монотонных базисах, т.е. в базисах, замыкание которых относительно операции суперпозищии совпадает с множеством всех монотонных булевых функций. В [14] он доказал следующее утверждение.

ТЕОРема 2.3. Пусть Еீ- произвольный конечный полный монотонный базис. тогда

$$
L_{\mathscr{E}}^{M}(n)-\frac{\rho(\mathscr{E})}{n}\left(\begin{array}{c}
n \\
\lfloor n / 2\rfloor
\end{array}\right) \leqslant O\left(\left(\begin{array}{c}
n \\
\lfloor n / 2\rfloor
\end{array}\right) \frac{\log n}{n}\right) .
$$

При доказательстве этой теоремы А.Е. Андреев предложил метод синтеза булевых схем в полных монотонных базисах, основанный на использовании специальных подклассов монотонных булевых функций, образующих булевы алгебры с операциями \&, $\vee$, а также новой модификации метода Клейтмана перечисления.

Среди булевых схем особьй интерес представляют схемы, в которых выход каждого элемента подключен не более чем к одному входу какого-либо элемента. Такие схемы часто назьвают суперпозициями. Поскольку понятие суперпозиции используется для формул, мы считаем, что такие схемы лучше назьвать булевыми схемами без ветвлений.

Обозначим через $L_{1, \mathscr{E}}^{M}(n)$ функцию Шеннона для сложности булевых схем без ветвлений в базисе $\mathscr{E}$, вычисляющих функции из $M(n)$.

Н. П. Редькин исследовал поведение функции $L_{1, \mathscr{E}}^{M}(n)$. Он доказал [251], что

$$
L_{1,\{\&, \vee\}}^{M}(n)=\Theta\left(\left(\begin{array}{c}
n \\
\lfloor n / 2\rfloor
\end{array}\right) / \log n\right) .
$$

Наконец, А.Е. Андреев [10] сфформулировал (без доказательства) следуюшее утверждение. Пусть $\mathscr{E}$ - произвольньй конечный полный базис для класса всех булевых функций. Тогда при $n \rightarrow \infty$

$$
L_{1, \mathscr{E}}^{M}(n) \sim \rho(\mathscr{E})\left(\begin{array}{c}
n \\
\lfloor n / 2\rfloor
\end{array}\right) / \log _{2} n .
$$




\section{§ 2.3. Сложность контактных схем}

Двухполюсной контактной схемой назьвается неориентированньй граф̆ с двумя выделенными вершинами (полюсами), каждому ребру которого приписана одна из букв $x_{1}, \bar{x}_{1}, \ldots, x_{n}, \bar{x}_{n}$, где $x_{1}, \ldots, x_{n}$ - булевы переменные, а $\bar{x}_{1}, \ldots, \bar{x}_{n}$ - их отрицания. Ребро с приписанной ему переменной $x_{i}$ (или $\bar{x}_{i}$ ) называется замыкаюшим (размыкающим) контактом.

Простая цепь между полюсами $a$ и $b$ в контактной схеме $S$ замкнута, если замкнуты все ее контакты, и разомкнута, если разомкнут хотя бы один ее контакт.

Проводимостью между $a$ и $b$ назьвается совокупность всех замкнутых простых цепей между $a$ и $b$. Таким образом, проводимость между полюсами $a$ и $b$ в $S$ задается булевой функцией $f\left(x_{1}, \ldots, x_{n}\right)$ такой, что на наборе $\left(\sigma_{1}, \ldots, \sigma_{n}\right)$ из $E^{n}$ функция $f\left(x_{1}, \ldots, x_{n}\right)$ равна 1 тогда и только тогда, когда между $a$ и $b$ в $S$ есть простая цепь, в которой не встречается контакт $x_{i}$, если $\sigma_{i}=0$, и отсутствует контакт $\bar{x}_{i}$, если $\sigma_{i}=1$, $1 \leqslant i \leqslant n$.

Поскольку ниже рассматриваются только двухполюсные схемы, слово “двухполюсная" будем опускать.

Контактная схема назьвается монотонной, если в ней отсутствуют размькающие контакты. Контактная схема называется ориентированной, если граф такой схемы является ориентированным. В ориентированной контактной схеме каждый контакт имеет одностороннюю проводимость, один полюс является входным, а другой - выходным.

Под сложностью контактной схемы понимается число контактов в ней.

Первые серьезные исследования по синтезу контактных схем были проведены К. Шенноном [276]. Впоследствии эти исследования были продолжены в работе [255] для параллельно-последовательных контактных схем.

Изложим результаты по сложности контактных схем, вычисляюших монотонные булевы функции.

Для булевых функций от небольшого числа переменных имеются каталоги минимальных контактных схем, вычисляющих эти функции. Например, Ю. Л. Васильев [314] привел каталог минимальных контактных схем, вычисляющих все булевы функции от четырех переменных (аналогичный каталог имеется в [211]). Н.А. Карпова в [119] привела список всех 210 типов монотонных булевых функций от пяти переменных и для каждого типа указаламинимальную контактную схему. Метод доказательства минимальности приведенных схем в основном сводится к получению нижних оценок сложности и отысканию схемы с такой сложностью.

Имеется несколько работ (см., например, [36], [314]), в которых доказана минимальность контактных схем, вычисляющих конкретные монотонные булевы функции от небольшого числа переменных.

Обозначим через $L_{k}^{M}(n)$ функцию Шеннона для сложности контактных схем, pеализующих функции из $M(n)$.

С. В. Яблонский [336], [337] установил, что $L_{k}^{M}(n)=o\left(2^{n} / n\right)$. Нижняя оценка для $L_{k}^{M}(n)$ вида

$$
L_{k}^{M}(n)>\frac{1}{n}\left(\begin{array}{c}
n \\
n / 2
\end{array}\right)\left(1-\frac{\log _{2} n}{n}\right)
$$

легко получается применением (2.2), а также теоремы 7 и леммы из [276]. 
Нетривиальная верхняя оценка для $L_{k}^{M}(n)$ была получена Ж. Анселем в 1966 г. В статье [97] он доказал, что

$$
L_{k}^{M}(n)<\frac{\sqrt{2 \log _{2} n}}{n}\left(\begin{array}{c}
n \\
n / 2
\end{array}\right)\left(1+O\left(\frac{1}{\log n}\right)\right) .
$$

Аналогичное утверждение для контактно-вентильных схем (в настояшей статье они не определены) было получено Э.И. Нечипоруком в 1962 г. [202] как следствие его результата о сложности вычисления булевых матриц вентильными схемами.

Н. П. Редькин [251] оценку (2.9) улучшил до следующей:

$$
L_{k}^{M}(n)<4.2\left(\begin{array}{c}
n \\
n / 2
\end{array}\right) / n
$$

Из (2.8) и (2.10) следует

Teopema 2.4. Прu $n \rightarrow \infty$

$$
L_{k}^{M}(n)=\Theta\left(\left(\begin{array}{c}
n \\
n / 2
\end{array}\right) / n\right) .
$$

Информация о структуре типичных функщий из $M(n)$, приведенная в первой главе, оказалась полезной при изучении сложности вычислений функций из $M(n)$ схемами различных типов.

Н.Н. Нурмеев [213] воспользовался этой информацией для модификации вариационного приншипа Нигматуллина [208] (см. также [210]) при исследовании сложности выгисления функций из $M(n)$ контактными схемами, параллельно-последовательными контактными схемами и некоторьми другими схемами.

Он доказал [213], что в $M(n)$ имеется подмножество $M^{\prime}(n)$ такое, что $|M(n)| \sim$ $\left|M^{\prime}(n)\right|$ при $n \rightarrow \infty$, и сложности минимальных контактных схем, вычисляющих функции из $M^{\prime}(n)$, асимптотически равны значению некоторой функции $g(n)$. При этом явньй вид функции $g(n)$ не указан (теорема существования).

Среди контактных схем особый интерес представляют параллельно-последовательные контактные схемы, которые часто называются $\pi$-схемами. Такие схемы можно определить индуктивно следуюшим образом:

a) одиночный контакт $(a, b)$ с полюсами $a$ и $b$ есть $\pi$-схема;

b) если $S_{1}\left(a_{1}, b_{1}\right)$ и $S_{2}\left(a_{2}, b_{2}\right)$ суть $\pi$-схемы, то схема $S(a, b)$, полученная отождествлением (склеиванием) полюсов $a_{1}$ с $a_{2}$ и $b_{1}$ с $b_{2}$, есть $\pi$-схема (параллельное соединение $\pi$-схем $S_{1}$ и $\left.S_{2}\right)$ с полюсами $a=a_{1}=a_{2}$ и $b=b_{1}=b_{2}$;

c) схема $S_{1}\left(a_{1}, b_{2}\right)$, полученная склеиванием полюсов $a_{2}$ и $b_{1}$ схем $S_{1}\left(a_{1}, b_{1}\right)$ и $S_{2}\left(a_{2}, b_{2}\right)$, есть $\pi$-схема (последовательное соединение $\pi$-схем $S_{1}$ и $\left.S_{2}\right)$ с полюсами $a_{1}$ и $b_{2}$;

d) других $\pi$-схем нет.

Укажем на одну связь между $\pi$-схемами и формулами в базисе $\{\vee, \&,-\}$.

Используя соотношения

$$
\overline{x_{1} \vee x_{2}}=\bar{x}_{1} \& \bar{x}_{2}, \quad \overline{x_{1} \& x_{2}}=\bar{x}_{1} \vee \bar{x}_{2}, \quad \overline{\bar{x}}=x,
$$


от произвольной формулы в базисе $\{\vee, \&,-\}$ можно перейти к формуле с тем же числом переменных, в которой знаки отрицания встречаются только над символами переменных. Вместе с тем известно, что между такими формулами и $\pi$-схемами существует такое соответствие, при котором соответствующие друг другу $\pi$-схема и формула реализуют одну и ту же функцию и имеют одинаковую сложность (под сложностью формулы понимается число символов переменных в ней).

Сложность $\pi$-схем, реализуюших функции из $M(n)$, изучалась многими исследователями.

Обозначим через $L_{\pi}^{+}(n)$ минимальное число контактов, достаточное для вычисления любой функции из $M(n) \pi$-схемами, содержащими только замыкающие контакты (функция Шеннона).

Нижняя оценка для $L_{\pi}^{+}(n)$ вида

$$
L_{\pi}^{+}(n)>\frac{1}{\log _{2} n}\left(\begin{array}{c}
n \\
n / 2
\end{array}\right)\left(1-\frac{3}{\log _{2} n}\right)
$$

легко устанавливается с использованием неравенства (2.2) и полученной в работе [255] верхней оценки для числа $\pi$-схем, вычисляюших булевы функции от $n$ переменных.

Верхние оценки для $L_{\pi}^{+}(n)$ таковы. Сначала Н. Н. Субоч [292] доказала, что

$$
L_{\pi}^{+}(n)<O\left(\left(\begin{array}{c}
n \\
n / 2
\end{array}\right)\right)
$$

Затем эта оценка была улучшена Н. П. Редъкиным в 1979 г. Он доказал [251], что

$$
L_{\pi}^{+}(n)<O\left(\left(\begin{array}{c}
n \\
n / 2
\end{array}\right) / \log n\right)
$$

Из (2.11) и (2.12) следует, что

$$
L_{\pi}^{+}(n)=\Theta\left(\left(\begin{array}{c}
n \\
n / 2
\end{array}\right) / \log n\right)
$$

Н.Н. Нурмеев [213] установил следуюший факт. В $M(n)$ имеется подмножество $M^{\prime \prime}(n)$ такое, что $|M(n)| \sim\left|M^{\prime \prime}(n)\right|$ при $n \rightarrow \infty$, и сложности $\pi$-схем, вычисляюших функции из $M^{\prime \prime}(n)$, асимптотически равны значению некоторой функции $h(n)$. При этом явный вид функции $h(n)$ не указан (теорема существования).

В [293] изучалась сложность монотонных контактных схем, вьгисляющих функции из $M(n)$ с небольшим числом нижних единиц.

\section{$\S$ 2.4. Сложность вычисления и задания монотонных булевых функций из замкнутых классов}

Сначала осветим результаты, относяшиеся к поведению функции Шеннона, когда монотонные булевы функции из каждого замкнутого класса вычисляются булевыми схемами в произвольном конечном полном базисе. (Все замкнутые классы монотонных булевых функций перечислены в $\S 1.4$.) 
Пусть $\mathscr{E}$ - произвольный конечный полный базис (для множества всех булевых функций), $H$ - один из замкнутых классов монотонных булевых функций и $H(n)$ - совокупность функщий из $H$ от $n$ переменных $x_{1}, \ldots, x_{n}$. Обозначим через $L_{\mathscr{E}}(H(n))$ минимальное число такое, что любая функция из $H(n)$ может быть вычислена булевой схемой в базисе $\mathscr{E}$, сложность которой не превосходит $L_{\mathscr{E}}(H(n))$.

Исследование асимптотического поведения функции $L_{\mathscr{E}}(H(n))$ для всех замкнутых классов монотонных булевых функций было проведено А. Б. Угольниковым [302]. Им были найдены асимптотики для $L_{\mathscr{E}}\left(A_{1}(n)\right)=L_{\mathscr{E}}(M(n))$ и $L_{\mathscr{E}}\left(D_{2}(n)\right)$ (асимптотика для $L_{\mathscr{E}}(M(n))$ приведена вьше в теореме 2.1). Асимптотики для $L_{\mathscr{E}}(H(n))$ в случае остальных замкнутых классов монотонных булевых функций установлены в [304] (см. также [303] и [306]). В итоге А.Б. Угольниковым была доказана следующая теорема.

ТеОрема 2.5. 1. Если $H(n)$ является одним из классов $A_{i}(n), 1 \leqslant i \leqslant 4$, или $F_{j}^{2}$, əде $j=2,3,6,7$, mo

$$
L_{\mathscr{E}}(H(n)) \sim \rho\left(\begin{array}{c}
n \\
n / 2
\end{array}\right) / n .
$$

2. $Е с л и H(n)$ является одним из классов $D_{2}(n), F_{i}^{\infty}$ или $F_{i}^{m}$, где $i=2,3,6,7$ $u m=3,4, \ldots, \infty, m o$

$$
L_{\mathscr{E}}(H(n)) \sim \frac{1}{2} \rho\left(\begin{array}{c}
n \\
n / 2
\end{array}\right) / n .
$$

Теперь рассмотрим сложность неявной и параметрической представимости монотонных булевых функщий из замкнутых классов. Такие представления булевых функций и функций многозначной логики впервые изучал А. В. Кузнецов [171]. Позднее неявное и параметрическое представление булевых функций изучал О.М. Касим-Заде [120]-[122]. Для булевых функций эти понятия определяются следующим образом.

Пусть $A$ - произвольное множество (система) булевых функций, $X=\left\{x_{1}, x_{2}, \ldots\right\}$ - бесконечное множество булевых переменных и $z$ - булева переменная, не принадлежащая множеству $X$. Переменные из $X$ называются основнылми, а $z$ - выделенной переменной.

Cистемой неявных уравнений над $A$ назьвается произвольная система уравнений вида

$$
\begin{aligned}
& \Phi_{1}\left(x_{1}, \ldots, x_{n}, z\right)=\Psi_{1}\left(x_{1}, \ldots, x_{n}, z\right), \\
& \ldots \ldots \ldots \ldots \ldots \ldots \ldots \ldots \ldots \ldots \ldots \ldots \ldots . . \ldots \ldots \\
& \Phi_{s}\left(x_{1}, \ldots, x_{n}, z\right)=\Psi_{s}\left(x_{1}, \ldots, x_{n}, z\right),
\end{aligned}
$$

где $\Phi_{1}, \ldots, \Phi_{s}, \Psi_{1}, \ldots, \Psi_{s}$ - формулы над $A$, состояшие из символов функций, входяших в систему $A$, символов основных переменных и выделенной переменной $z$.

Говорят, что система уравнений (2.13) неявно задает булеву функцию $f\left(x_{1}, \ldots, x_{n}\right)$, если при любых значениях основных переменных $x_{1}, \ldots, x_{n}$ система (2.13) имеет единственное решение относительно выделенной переменной $z$ и $z=f\left(x_{1}, \ldots, x_{n}\right)$.

Пусть $f$ - булева функция, неявно представимая над $A$ системой уравнений вида (2.13). Обозначим через $m_{A}(f)$ наименьшее число уравнений вида $(2.13)$ над $A$, достаточное для неявного представления функции $f$. Пусть $m_{A}(n)=\max m_{A}(f)$, где максимум берется по всем булевым функциям $f$ от $n$ переменных, неявно представимым над $A$ системами уравнений вида (2.13). 
Далее, пусть $A$ - произвольная система булевьх функций, $X=\left\{x_{1}, x_{2}, \ldots\right\}$ и $Y=$ $\left\{y_{1}, y_{2}, \ldots\right\}$ - непересекаюшиеся множества булевых переменных и $z$-булевапеременная, не входящая в $X \cup Y$. Переменные из $X$ назьваются основнылми переменными, из $Y$ - внутренними переменными, $z$ - выделенной переменной.

Cистемой параметрических уравнений над $A$ называется произвольная система уравнений вида

$$
\begin{aligned}
& \Phi_{1}\left(x_{1}, \ldots, x_{n}, y_{1}, \ldots, y_{p}, z\right)=\Psi_{1}\left(x_{1}, \ldots, x_{n}, y_{1}, \ldots, y_{p}, z\right)
\end{aligned}
$$

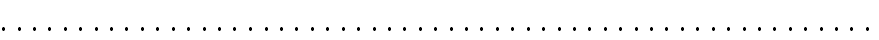

$$
\begin{aligned}
& \Phi_{s}\left(x_{1}, \ldots, x_{n}, y_{1}, \ldots, y_{p}, z\right)=\Psi_{s}\left(x_{1}, \ldots, x_{n}, y_{1}, \ldots, y_{p}, z\right) \text {, }
\end{aligned}
$$

где $\Phi_{1}, \ldots, \Phi_{s}, \Psi_{1}, \ldots, \Psi_{s}$ - формулы над $A$, состояшие из символов функций, входяших в систему $A$, и символов переменных $x_{1}, \ldots, x_{n}, y_{1}, \ldots, y_{p}, z$.

Говорят, что система (2.14) параметрически представляет (задает) булеву функцию $f\left(x_{1}, \ldots, x_{n}\right)$, если при любых значениях основных переменных $x_{1}, \ldots, x_{n}$ система (2.14) имеет по крайней мере одно решение относительно переменных $y_{1}, \ldots, y_{p}, z$, причем при фиксированных значениях $y_{1}, \ldots, y_{p}$ система имеет единственное решение относительно выделенной переменной $z=f\left(x_{1}, \ldots, x_{n}\right)$.

Пусть $f$ - булева функция, параметрически представимая над $A$ системой уравнений вида (2.14). Обозначим через $M_{A}(f)$ наименьшее число уравнений вида $(2.14)$ над $A$, достаточное для параметрического представления функции $f$. Пусть $M_{A}(n)=$ $\max M_{A}(f)$, где максимум берется по всем булевым функциям $f$ от $n$ переменных, параметрически представимым над $A$ системами уравнений вида (2.14).

Решение проблемы параметрической представимости булевых функций дано А.В. Кузнецовым в работе [171], в которой для каждой системы $A$ булевых функций описаны функции, параметрически представимые над $A$. Оказалось, что параметрическая представимость значительно сильнее представимости суперпозициями.

Например, над каждой системой $A$, замькание которой по суперпозиции совпадает с классом всех монотонных булевых функций, параметрически представима любая булева функция.

О.М. Касим-Заде [120] (см. также [122]) установил, что в двузначной логике неявная представимость эквивалентна параметрической. В [122] (см. также [120] и [121]) им доказана следуюшая теорема.

Теорема 2.6. 1. Если замыкание (относительно суперпозиции) системы $A$ совпадает с замкнутым классом $A_{1}$ (т.е. с множсеством всех монотонных булевых функиий), то при любом $n, n \geqslant 1$, справедливь равенства

$$
m_{A}(n)=\lfloor(n+1) / 2\rfloor+1, \quad M_{A}(n)=2 .
$$

2. Если замыкание (относительно суперпозичии) системы А совпадает либо с замкнутым классом $A_{2}$, либо с замкнутым классом $A_{3}$, то при любом $n, n \geqslant 1$, справедливы равенства

$$
m_{A}(n)=\lceil(n+1) / 2\rceil, \quad M_{A}(n)= \begin{cases}1 & \text { nри } n=1 \\ 2 & \text { nри } n \geqslant 2 .\end{cases}
$$


3. Если замыкание (относительно суперпозиции) системы А совпадает с замкнутыл классом $A_{4}$, то при любом $n, n \geqslant 1$, справедливь равенства

$$
m_{A}(n)=\lfloor(n+1) / 2\rfloor, \quad M_{A}(n)= \begin{cases}1 & \text { nри } n \leqslant 2, \\ 2 & \text { nри } n \geqslant 3 .\end{cases}
$$

4. Если замыкание (относительно суперпозиции) системы $A$ совпадает с замкнутылм классом $D_{2}$ или с одним из замкнутых классов $F_{i}^{m}$, где $i=2,3,6,7 u$ $m=2,3, \ldots, \infty$, то при $n \rightarrow \infty$ справедливь соотношения

$$
m_{A}(n)=\Omega(n \log n), \quad M_{A}(n)=\Omega(n) .
$$

\section{$\S$ 2.5. Симметрические монотонные булевы функции}

Одним из простейших классов монотонных булевых функций является класс симметрических функций.

Булева функция назьвается симметрической, если она принимает одно и то же значение на всех наборах значений своих переменных, содержащих одинаковое число единиц.

Обозначим через $f_{r}\left(x_{1}, \ldots, x_{n}\right)$ симметрическую функцию из $M(n)$, которая равна 1 на любом наборе значений переменных $x_{1}, \ldots, x_{n}$, содержашем не менее $r$ единиц, и равна 0 на остальных наборах значений переменных, $1 \leqslant r \leqslant n$.

Сложность минимальных булевых схем, формул и контактных схем, вычисляющих функции $f_{r}\left(x_{1}, \ldots, x_{n}\right), 2 \leqslant r \leqslant n$, исследовалась во многих работах. Начнем с рассмотрения функции $f_{2}\left(x_{1}, \ldots, x_{n}\right)$.

В 1956 г. В.К. Коробков [143] предложил метод построения формул в базисе $\{\&, \vee,-\}$ (следовательно, и $\pi$-схем), задаюших функции $f_{r}\left(x_{1}, \ldots, x_{n}\right), 1 \leqslant r \leqslant n$. $\mathrm{C}$ помошюю этого метода удается строить формулу, которая задает функции $f_{2}\left(x_{1}, \ldots, x_{n}\right)$ и имеет сложность не более $O(n \log n)$.

О.Б. Лупанов [182] доказал, что в произвольной контактной схеме, которая вычисляет функцию $f_{2}\left(x_{1}, \ldots, x_{n}\right)$, содержится $\Omega\left(\frac{n \log n}{\log \log n}\right)$ контактов.

Р.Е. Кричевский [167], [168] доказал, что число контактов в любой $\pi$-схеме, вычисляющей функцию $f_{2}\left(x_{1}, \ldots, x_{n}\right)$, не меньше $\frac{1}{4} n \log _{2} n$. С точностью до порядка эта нижняя оценка совпадает с верхней оценкой Коробкова из [143]. Главное в методе Кричевского - доказательство того, что среди минимальных $\pi$-схем имеется монотонная $\pi$-схема.

Позднее Ж. Ансель [93] показал, что в минимальной монотонной контактной схеме, которая вычисляет функцию $f_{2}\left(x_{1}, \ldots, x_{n}\right)$, содержится не менее $\left\lfloor n \log _{2} n\right\rfloor$ контактов. Используя результат Анселя [94] и лемму 1 из [169], Р.Е. Кричевский [170] доказал следующее утверждение.

ТЕОРема 2.7. При любом $n \geqslant 2$ число контактов в минимальной монотонной контактной схеме, вьчисляющей функиию $f_{2}\left(x_{1}, \ldots, x_{n}\right)$, в точности равно $n\left\lfloor\log _{2} n\right\rfloor+2\left(n-2^{\left\lfloor\log _{2} n\right\rfloor}\right)$. Одной из таких схем является $\pi$-схема, которая описана в [143].

Вскоре аналогичньй результат для монотонных контактных схем получил Ж. Ансель [95]. 
Изложение перечисленных результатов имеется в монографиях [210], [268].

Сложность вьгисления функции $f_{2}\left(x_{1}, \ldots, x_{n}\right)$ также изучалась в [280].

Теперь изложим результаты, относящиеся к $f_{r}\left(x_{1}, \ldots, x_{n}\right)$ при произвольном $r$. К. Шеннон [276; теорема 16] доказал, что в минимальной контактной схеме, которая вычисляет функцию $f_{r}\left(x_{1}, \ldots, x_{n}\right), 2 \leqslant r \leqslant n$, содержится не более $n^{2}-n+2$ контактов. Доказательство основано на разложении функции $f_{r}\left(x_{1}, \ldots, x_{n}\right)$ по элементарным периодическим функциям. Позднее О. Б. Лупанов [184] усилил этот результат. Для каждого $r, 2 \leqslant r \leqslant n$, он предложил конкретную контактную схему, которая вычисляет функцию $f_{r}\left(x_{1}, \ldots, x_{n}\right)$ и содержит не более $O\left(n^{3 / 2}\right)$ контактов. Доказательство основано на разложении функции $f_{r}\left(x_{1}, \ldots, x_{n}\right)$ на элементарные периодические функции.

Затем эта оценка была улучшена Е.Г. Красулиной [165] (подробное изложение в [166]). Используя большее число элементарных периодических функций, на которые может быть разложена функция $f_{r}\left(x_{1}, \ldots, x_{n}\right)$, она показала, что верхнюю оценку $O\left(n^{3 / 2}\right)$ можно заменить на величину $O\left(\left(n \log ^{4} n\right) /(\log \log n)^{2}\right)$.

А. А. Марков [191] исследовал сложность контактно-вентильных схем, вычисляюших функцию $f_{r}\left(x_{1}, \ldots, x_{n}\right), 2 \leqslant r \leqslant n$. Им была доказана следуюшая теорема.

ТЕОРема 2.8. Пусть контактно-вентильная схема вычисляет $f_{r}\left(x_{1}, \ldots, x_{n}\right)$, $2 \leqslant r \leqslant n$, и содержит минимально возможсное число контактов. Тогда это число равно $r(n-r+1)$.

При этом А.А. Марков указал достаточно регулярную схему с таким числом контактов.

О.Б. Лупанов [183; теорема 3.1] показал, что при любом $r, 2 \leqslant r \leqslant n$, функция $f_{r}\left(x_{1}, \ldots, x_{n}\right)$ может быть вычислена такой булевой схемой в произвольном полном базисе, в которой содержится не более $O(n)$ (не более $5 n(1+o(1)))$ элементов (см. также [184]). Отсюда и из теоремы 2.7 следует, что сложность минимальной контактной схемы и сложность булевой схемы, вычисляющих одну и ту же функцию, могут существенно различаться.

Позднее Э. А. Ламанья и Дж. Сэвидж [173] показали, что каждая булева схема в базисе $\{\&, \bigvee\}$, в которой на выходах $n$ элементов вычисляются все ненулевые симметрические функции из $M(n)$, содержит $\Omega(n \log n)$ элементов.

Рассмотрим результаты, связанные с оценками сложности минимальных формул, задающих функции $f_{r}\left(x_{1}, \ldots, x_{n}\right)$ при произвольном $r, 2 \leqslant r \leqslant n$. Первьй результат в этом направлении был получен В. К. Коробковым в 1956 году. Используя метод “деления пополам" (дихотомии), он показал [143], что любую симметрическую функцию от $n$ переменных (следовательно, любую функцию $f_{r}\left(x_{1}, \ldots, x_{n}\right), 1 \leqslant r \leqslant n$ ) можно задать такой формулой в базисе $\{\&, \vee,-\}$ (следовательно, $\pi$-схемой), сложность которой не превосходит $n^{\left(\frac{1}{2}+\varepsilon_{n}\right) \log _{2} n}$, где $\varepsilon_{n} \rightarrow 0$ при $n \rightarrow \infty$.

Впоследствии О.Б. Лупанов [184] усилил этот результат, показав, что оценка $n^{\left(\frac{1}{2}+\varepsilon_{n}\right) \log _{2} n}$ может быть понижена до величины $n^{\left(\frac{1}{2}+\varepsilon_{n}\right) \log _{2} \log _{2} n}$.

Дальнейшие результаты содержатся в статьях Л. С. Хасина [129]-[131]. В [129] он доказал сушествование формулы в базисе $\{\&, \vee\}$, которая задает функцию $f_{r}\left(x_{1}, \ldots, x_{n}\right), 2 \leqslant r \leqslant n$, и имеет сложность не более $2^{\left(\log _{2} r\right)^{2}\left(1+\varepsilon_{r}\right) / 4} n \log _{2} n$, где $\varepsilon_{r} \rightarrow 0$ при $r \rightarrow \infty$. Предложенньй метод получения этой оценки является 
неконструктивным: доказано существование формулы указанной сложности, но сама формула не предъявлена.

Л. С. Хасин [130] предложил эффективньй метод построения формулы в базисе $\{\&, \vee\}$, которая задает функции $f_{r}\left(x_{1}, \ldots, x_{n}\right)$ при $r \geqslant 3$ и имеет сложность не более $\frac{n\left(\log _{2} n\right)^{r-1}}{\left(\log _{2} \log _{2} n\right)^{r-2}}$.

Наконец, в [131] он описал метод построения формулы в базисе $\{\&, \vee,-\}$ сложности не более $\Omega(n \log n \log \log n)$, которая задает функцию $f_{3}\left(x_{1}, \ldots, x_{n}\right)$, и формулы сложности не более $O\left(n(\log n)^{\log _{2}(r-1)}\right)$, задающей функцию $f_{r}\left(x_{1}, \ldots, x_{n}\right)$ при $r \geqslant 4$.

В 1972 г. В.М. Храпченко [132] доказал, что любая симетрическая булева функция от $n$ переменных (в частности, любая функция $f_{r}\left(x_{1}, \ldots, x_{n}\right)$ ) может быть реализована $\pi$-схемой (следовательно, может быть задана формулой в базисе $\{\&, \vee,-\})$, сложность которой не превосходит $O\left(n^{4.93}\right)$.

В дальнейшем были получены следуюшие результаты. Л. Вальянт в [308] показал, что при любом четном $n$ функция $f_{n / 2}\left(x_{1}, \ldots, x_{n}\right)$ может быть задана формулой в базисе $\{\&, \vee\}$, размер которой не превосходит величины $O\left(n^{5.3}\right)$ (доказательство также имеется в [55; с. 335-339]). М. С. Патерсон [218] предложил метод построения формул, задающих функцию $f_{r}\left(x_{1}, \ldots, x_{n}\right), 2 \leqslant r \leqslant n$, и имеюших сложность не более $O\left(n(\log n)\left(\log _{2} \log _{2} n\right)^{r-2}\right)$.

Дж. Фридман [70], [71] доказал, что при любом $r, 1 \leqslant r \leqslant n$, функция $f_{r}\left(x_{1}, \ldots, x_{n}\right)$ может быть задана формулами в базисе $\{\&, \vee\}$ размера $\Theta\left(r^{12.6} n \log n\right)$ без явного указания самой формулы.

С. А. Ложкин и А. А. Семенов [177] доказали следующее утверждение. При фиксированном $r$ функцию $f_{r}\left(x_{1}, \ldots, x_{n}\right)$ можно задать формулой в базисе $\{\&, \vee\}$, которая имеет сложность не более $O\left(r^{5.3} n \log n\right)$.

Р. Боппана [27], [29] доказал сушествование такой формулы в базисе $\{\&, \vee\}$, которая задает функцию $f_{r}\left(x_{1}, \ldots, x_{n}\right)$ и имеет сложность не более $O\left(r^{4.3} n \log n\right)$.

В статье [52] рассматривалось вычисление функций $f_{r}\left(x_{1}, \ldots, x_{n}\right)$ монотонными контактньми схемами. В ней доказано, что функция $f_{\lceil n / 2\rceil}\left(x_{1}, \ldots, x_{n}\right)$ может быть вычислена схемой сложности не более $O\left(n^{4.99}\right)$, а любая функция $f_{r}\left(x_{1}, \ldots, x_{n}\right)$, $2 \leqslant r \leqslant n$, может быть вычислена схемой сложности не более $O\left(n^{3.99} n \log n\right)$. Доказательства обоих утверждений неконструктивны.

В статье [92] показано, что функция $f_{r}\left(x_{1}, \ldots, x_{n}\right), 2 \leqslant r \leqslant n-1$, может быть вычислена ориентированной монотонной контактной схемой, сложность которой не превосходит $O(r(n-r+2) \log (n-r+2))$. Эта оценка почти оптимальна при небольших $r$, поскольку в [230] (см. также [233]) показано, что величина $O(r n \log (n /(r-1)))$ является нижней оценкой. Кроме того, в [92] показано, что сложность любой монотонной контактной схемы, вычисляющей функщию $f_{n-1}\left(x_{1}, \ldots, x_{n}\right)$, paвна $\Omega(n \log \log \log n)$.

М.И. Гринчук [87] описал метод построения булевой схемы в базисе $\{\&, \vee\}$ сложности не более $r n+O\left(n^{1-1 / r}\right)$, вычисляюшей функцию $f_{r}\left(x_{1}, \ldots, x_{n}\right), 2 \leqslant r \leqslant n$. Эта оценка является асимптотически точной при $r=2$ не только в базисе $\{\&, \vee\}$, но и в базисе, состояшем из всех двуместных булевых функций.

М. Фишер, А. Мейер и М. Патерсон [68] предложили новый метод получения нижних оценок сложности булевых функций без ветвлений (формул) в базисе, состоящем из всех булевых функций от двух переменных. В частности, они показали, что в любой схеме такого вида, которая вычисляет функцию $f_{\lfloor n / 2\rfloor+1}\left(x_{1}, \ldots, x_{n}\right)$, 
содержится $\Omega(n \log n)$ элементов. (Описание метода имеется в [210; с. 164-181].)

Альтернативньй вариант последнего результата опубликован в [133].

Аналогичная оценка в случае произвольного конечного полного базиса получена Д. Ю. Черухиным [39].

Другие результаты по этой теме содержатся в статьях [95], [136], [3], [26], [53], [229], [54], [28], [141], [104], [105], [88], [234], [235] (ссылки приведены в хронологическом порядке).

\section{§ 2.6. Влияние отрицаний на сложность вычислений монотонных булевых функций}

Известно, что любую монотонную булеву функцию можно вычислить монотонной контактной схемой и булевой схемой в полном монотонном базисе.

Оказывается, что некоторые такие функции можно вычислять более простыми контактными схемами общего вида, нежели монотонными контактными схемами. Например, Ю. Л. Васильев показал (см., например, [118], [182]), что в любой минимальной контактной схеме, вычисляющей функцию $f\left(x_{1}, \ldots, x_{6}\right)=\left(x_{1} \vee x_{2} \vee x_{5}\right)\left(x_{3} \vee x_{4}\right) \vee$ $\left(x_{1} \vee x_{2}\right) x_{6}$, обязательно содержатся размькающие контакты. В связи с этим возникает задача о сравнении сложности вычисления монотонных булевых функций такими схемами. Для точной постановки задачи используются следуюшие обозначения.

Пусть $f$ - произвольная монотонная булева функция. Обозначим через $L_{k}^{+}(f)$ $\left(L_{k}(f)\right)$ минимальное число контактов, достаточное для вычисления функции $f$ монотонной контактной схемой (произвольной контактной схемой). Пусть $\lambda_{k}(f)=$ $L_{k}^{+}(f) / L_{k}(f)$ и $\lambda_{k}(n)=\max \lambda_{k}(f)$, где максимум берется по всем функциям $f$ из $M(n)$. Задача состоит в исследовании поведения функции $\lambda_{k}(n)$ при $n \rightarrow \infty$.

Решением этой задачи занимались многие исследователи. Сначала Н. А. Карпова [118] показала, что $\lambda_{k}(n) \geqslant \frac{3}{2(1+3 /(2 n))}$ при любом $n$. Затем О. Б. Лупанов [182] усилил этот результат. Для функции $f_{2}\left(x_{1}, \ldots, x_{n}\right)=\bigvee_{1 \leqslant i<j \leqslant n} x_{i} x_{j}$ он доказал, что

$$
\lambda_{k}\left(f_{2}\left(x_{1}, \ldots, x_{n}\right)\right)=\Omega\left(\frac{\log n}{\log \log n}\right) .
$$

Из этого результата следует, что некоторые монотонные булевы функции могут быть вычислены произвольными контактными схемами существенно проще, чем монотонными контактными схемами.

О.Б. Лупанов [184] показал, что $\lambda_{k}(n)=\Omega(\sqrt{n})$. Наконец, Е.Г. Красулина [166] (см. также [165]) доказала, что

$$
\lambda_{k}(n)=\Omega\left(n(\log \log n)^{2} / \log ^{4} n\right) .
$$

Далее обозначим через $L_{\pi}^{+}(f)$ минимальное число контактов, достаточное для вычисления монотонной булевой функции $f$ монотонной параллельно-последовательной контактной схемой (т.е. монотонной $\pi$-схемой). Пусть $\lambda_{\pi}(f)=L_{\pi}^{+}(f) / L_{\pi}(f)$ и $\lambda_{\pi}(n)=$ $\max \lambda_{\pi}(f)$, где максимум берется по всем функциям $f$ из $M(n)$. 
В [36] приведен пример такой монотонной булевой функции $f$ от 8 переменных, что $\lambda_{\pi}(f)>1$. В.Я. Пивкин [225] доказал, что при любом достаточно большом $n$ справедливо неравенство

$$
\lambda_{\pi}(n)>\frac{5}{4(1+6 /(n-5))} .
$$

Более высокая нижняя оценка для $\lambda_{\pi}(n)$ была получена Е. А. Окольнишниковой [214]. Она доказала, что

$$
\lambda_{\pi}(n)=\Omega\left(\frac{\log n}{\log \log n}\right) .
$$

$\mathrm{K}$ этому же направлению относится работа [215]. В ней для каждого $n$ указана функция $f \in M(n)$, обладающая следуюшим свойством: сложность минимальной формулы глубины 3 в базисе $\{\&, \vee,-\}$, которая задает функцию $f$, и сложность аналогичной формулы в базисе $\{\&, \vee\}$ различаются не менее чем в $\Omega\left(n^{1 / 4}\right)$ раз.

А. В. Чашкин [37] изучал влияние отрицаний на сложность вычисления частичных монотонных булевых функций контактными схемами, $\pi$-схемами и булевыми схемами в базисах $\{\&, \vee,-\}$ и $\{\&, \vee\}$.

После многих лет постепенного повышения нижних оценок для функций $\lambda_{k}^{+}(n)$, $\lambda_{\pi}^{+}(n)$ и аналогичных оценок для булевых схем в базисе $\{\&, \vee\}$ существенньй прорьв был сделан А.А. Разборовым в 1985 году. В работе [239] (подробное изложение в [240]; см. также [243]) он впервые получил сверхполиномиальные нижние оценки для сложности булевых схем в базисе $\{\&, \vee\}$, вычисляющих естественно возникаюшие монотонные булевы функции из двух последовательностей.

Почти одновременно А.Е. Андреев [9] (подробное изложение в [12]) для каждого $n$ установил нижнюю оценку вида $2^{(1 / 8)} n-o(1)$ для сложности булевых схем в базисе $\{\&, \vee\}$, вычисляющих индивидуальные монотонные булевы функции от $n$ переменных. (Более подробно см. $§ 2.8$.)

\section{$\S$ 2.7. Нижние оценки сложности схем, вычисляющих конкретные монотонные булевы функции}

Проблема нижних оценок сложности, имеющая более полувековую историю, - одна из наиболее трудных в дискретной математике. Она представляет большой интерес как для теории, так и для практики.

В прикладном плане решение проблемы нижних оценок сложности способствовало бы разработке более эффективных алгоритмов и созданию методики по оценке качества алгоритмов и программ.

Проблема нижних оценок сложности схем состоит в том, чтобы установить хорошие нижние оценки сложности вычисления конкретных булевых функций.

Несмотря на значительные усилия многих математиков на протяжении нескольких десятилетий нет заметного прогресса в решении этой проблемы. В случае полных базисов известны лишь линейные (относительно числа переменных вычисляемой функции) нижние оценки сложности минимальных булевых схем.

Для сложности контактных схем и булевых схем без ветвлений в произвольном конечном полном базисе, вычисляюших конкретные булевы функции (точнее, последовательности таких функций), известны более высокие нижние оценки. Впервые такие оценки (за исключением оценок сложности кратчайших дизъюнктивных нормальных 
форм) были получены Э. И. Нечипоруком в 1966 г. [205]. Обобщение и описание предложенного им метода, который применим только к булевым схемам без ветвлений, имеется в монографиях [210], [268] и [55; с. 281-286].

Более чем квадратичная (по числу переменных) нижняя оценка сложности минимальных булевых схем без ветвлений в базисе $\{\&, \vee,-\}$, вычисляющих конкретную булеву функцию от $n$ переменных, $n \geqslant 1$, впервые была получена А.Е. Андреевым в 1987 г. [13]. Эта оценка по порядку равна величине $\frac{n^{5 / 2}}{(\log n)^{1 / 2} \log \log n}$. Она была установлена на основе обобщения метода Б. А. Субботовской [294] с использованием идеи универсальной функции Э.И. Нечипорука [205]. Доказательство этой оценки имеется в монографиии [55].

Позднее оценка Андреева была повышена до величины порядка $n^{2.556}$ в [110] и до величины порядка $\frac{n^{3}}{(\log n)^{11 / 2} \log \log n}$ в [102].

Трудности решения проблемы нижних оценок сложности схем побудили рассматривать более слабые модели вычислений, а именно булевых схем с ограничениями: схемы в неполных базисах, схемы ограниченной глубины и т. д. В результате исследований математиков для таких схем удалось получить высокие нижние оценки их сложности. Первые такие оценки получены А. А. Разборовым [239], [240] и А. Е. Андреевым [9], [12] для сложности минимальных булевых схем в базисе $\{\&, \bigvee\}$, вычисляющих известные булевы функции.

Теперь дадим обзор нижних оценок сложности булевых схем в монотонных базисах, вычисляюших конкретные монотонные булевы функции, которые получены без использования идей А. А. Разборова и А.Е. Андреева.

Сначала осветим результаты, связанные со сложностью вычислений конъюнкции $K_{n}=x_{1} \& x_{2} \& \cdots \& x_{n}$ и дизъюнкции $D_{n}=x_{1} \vee x_{2} \vee \cdots \vee x_{n}$.

Е.П. Сопруненко [289] рассмотрела вычисление функций $K_{n}$ и $D_{n}$ булевыми схемами в базисе $\mathscr{E}$, состояшем из одной функции $f\left(x_{1}, x_{2}\right)=\bar{x}_{1} \vee \bar{x}_{2}$, известной под названием "штрих Шеффера". В [289] доказаны следующие утверждения:

1) минимальная булева схема в базисе $\mathscr{E}$, вычисляющая функцию $K_{n}$, состоит из $2 n-2$ элементов;

2) минимальная булева схема в базисе $\mathscr{E}$, вычисляющая функшию $D_{n}$, состоит из $3 n-3$ элементов.

K этому же направлению относится работа [212].

Э.И. Нечипорук в 1970 г. [207] указал первьй пример произвольно высоких степенных оценок сложности минимальных булевых схем без ветвлений в неполных базисах. Он рассмотрел базис $\mathscr{E}$, состоящий из одной функции

$$
\varphi_{l, m}\left(x_{1,1}, \ldots, x_{l, m}\right)=\bigvee_{i=1}^{l} \bigwedge_{j=1}^{m} x_{i, j},
$$

где $l \geqslant 2$ и $m \geqslant 2$ - произвольные натуральные числа, и показал, что булевы схемы без ветвлений в базисе $\mathscr{E}$, которые вычисляют функции $D_{n}$ и $K_{n}$, содержат

$$
L_{\mathscr{E}}\left(D_{n}\right)=\Omega\left(n^{\log _{2} m / \log _{2} l+1}\right)
$$


и

$$
L_{\mathscr{E}}\left(K_{n}\right)=\Omega\left(n^{\log _{2} l / \log _{2} m+1}\right)
$$

Элементов соответственно.

Из (2.15) и (2.16) следует, что для любого $c_{1}>0$ можно подобрать такие $l$ и $m$, что $L_{\mathscr{E}}\left(D_{n}\right)=\Omega\left(n^{c_{1}}\right)$, и для любого $c_{2}>0$ можно подобрать такие $l$ и $m$, что $L_{\mathscr{E}}\left(K_{n}\right)=$ $\Omega\left(n^{c_{2}}\right)$.

Доказательство неравенств (2.15) и (2.16) имеется также в [210].

В 1969 г. Э.И. Нечипорук [206] описал конкретную систему из $n$ различных дизъюнкций (в каждую дизъюнкцию входят некоторые переменные из множества $\left.\left\{x_{1}, \ldots, x_{n}\right\}\right)$, в которой любые две дизъюнкции имеют не более одной общей переменной, а $n=p^{2}$, где $p$ - любое простое число. Он показал, что каждая минимальная булева схема в базисе $\{\&, \vee\}$ с $n$ входами и $n$ выходами, вычисляющая функции этой системы, не содержит конъюнкторов и состоит из $n^{3 / 2}-n$ дизъюнкторов. Эта оценка является первой нелинейной нижней оценкой для сложности булевых схем в неполном базисе, вычисляющих системы конкретных (монотонных) булевых функций.

Используя метод Нечипорука, Э. А. Ламанья и Дж. Э. Сэвидж [173] и Р. Е. Тарьян [296] для каждого $n$ предложили другие системы $n$ монотонных булевых функций от $n$ одинаковых переменных и показали, что любая булева схема с $n$ входами и $n$ выходами в базисе $\{\&, \vee\}$, вычисляющая функции одной такой системы, содержит $\Omega\left(n^{3 / 2}\right)$ элементов.

У. Дж. Пауль [219] для каждого $n$ предложил систему $n$ монотонных булевых функций от $n$ одинаковых переменных и доказал следуюшие утверждения:

1) для почти каждого $n$ сложность любой булевой схемы с $n$ входами и $n$ выходами в базисе $\{\&, \vee\}$, вычисляющей функции этой системы, равна $\Omega\left(n^{2} / \log ^{3 / 2} n\right)$;

2) для любого $n$ сложность каждой булевой схемыв базисе $\{\&, \vee\}$, вычисляющей функции этой системы, по порядку не превосходит величины $n \log ^{2} n$.

Д. Ю. Григорьев [86] при каждом $n$ описал систему из таких $n-1$ дизьюнкций ( $n=2^{k}$, где $k$ - произвольное натуральное число), что каждая дизъюнкция содержит не более $k$ переменных из заданного множества $n$ переменных. Он показал, что минимальная булева схема с $n$ входами и $n-1$ выходами в базисе $\{\bigvee\}$, которая вычисляет дизъюнкции этой системы, содержит не менее $\frac{1}{2}(k-1)(n-1)$ и не более $2 k n$ элементов. Ясно, что при $n \rightarrow \infty$ нижняя и верхняя оценки сложности асимптотически равны $\frac{1}{2} n \log _{2} n$ и $2 n \log _{2} n$ соответственно.

Развив метод Нечипорука [206], К. Мельхорн [195] для каждого $n$ рассмотрел конкретную систему таких $n$ дизъюнкций, что в каждую дизъюнкцию входят некоторые переменные из множества $\left\{x_{1}, \ldots, x_{n}\right\}$, а любые $n+1$ дизъюнкций имеют не более $h$ общих переменных. Используя идею свободных входов из [317] и [318], он показал, что любая булева схема с $n$ входами и $n$ выходами в базисе $\{\&, \vee\}$, которая вычисляет дизъюнкции этой системы, содержит $\Omega\left(n^{5 / 3}\right)$ элементов. Аналогичный результат был получен Н. Пиппенджером [222]. Метод Нечипорука использовался в работах [320], [99] и [319].

Н. Блюм [24] (см. также [25]) рассмотрел семейство монотонных булевых функций $\left\{f_{0}, f_{1}, \ldots, f_{2 n-2}\right\}$ от переменных $x_{1}, \ldots, x_{n-1}$ и $y_{1}, \ldots, y_{n-1}$ таких, что при любом $k$, 
$0 \leqslant k \leqslant 2 n-2$,

$$
f_{k}=\bigvee_{r+s} x_{r} \& y_{s}
$$

Он показал, что в любой булевой схеме с $2 n$ входами и $2 n-1$ выходами в базисе $\{\&, \vee\}$, вычисляющей функции этой системы, содержится не менее $\frac{1}{16}\left(n^{4 / 3}-n\right)$ конъюнкторов. В [331] эта нижняя оценка была усилена и заменена на величину $n^{3 / 2}$.

Е. А. Окольнишникова [216] ввела в рассмотрение систему $n$ конкретных монотонных булевых функций от $n$ одинаковых переменных, где $n=p^{2}$, а $p$ - любое простое число. Она доказала, что любая булева схема с $n$ входами и $n$ выходами в базисе $\{\&, \vee, 0,1\}$, которая вычисляет функции этой системы, содержит $\Omega\left(n^{2}\right)$ элементов.

Теперь перейдем к изложению результатов, относяшихся к сложности булева умножения булевых матрищ, т.е. матрищ, состояших из нулей и единиц.

Пусть $A=\left(a_{i j}\right)$ и $B=\left(b_{i j}\right)$ - булевы матрицы размера $m \times k$ и $k \times l$ соответственно. Булевьм произведением матрищ $A$ и $B$ называется матрица $C=\left(c_{i j}\right)$ размера $m \times l$ такая, что при любых $i$ и $j, 1 \leqslant i \leqslant m, 1 \leqslant j \leqslant l$, элемент $c_{i j}$ определяется следующим образом:

$$
c_{i j}=\bigvee_{v=1}^{k} a_{i v} \& b_{v j} .
$$

При вычислении $c_{i j}$ рассматриваются $i$-я строка матрицы $A$ и $j$-й столбец матрицы $B$ и выясняется, имеют ли эти два вектора обшую единичную компоненту.

В.P. Пратт доказал в [228] (см. также [227]), что минимальная булева схема с $2 n^{2}$ входами и $n^{2}$ выходами в базисе $\{\&, \vee\}$, вычисляющая булево произведение двух произвольных квадратных матриц порядка $n$, содержит не менее $\frac{1}{2} n^{3}$ конъюнкторов.

Используя идею из [228], М. С. Патерсон [217] и вскоре К. Мельхорн и З. Галил [197] (анонсировано в [196]) доказали, что минимальная булева схема с $m k+m l$ входами и $k l$ выходами в базисе $\{\&, \vee\}$, вычисляющая булево произведение любых двух булевых матриц размера $m \times k$ и $k \times l$, содержит $m k l$ конъюнкторов и $m l(k-1)$ дизъюнкторов. Кроме того, в этих работах показано, что всякая минимальная булева схема эквивалентна (с точностью до порядка вьполнения операций и перестановки входов) схеме, вычисляющей булево произведение согласно его определению. В частности, при $m=k=l$ такая схема содержит $n^{3}$ конъюнкторов и $n^{3}-n^{2}$ дизъюнкторов.

В то же время, переложив на язык схем известный результат В. Штрассена [291] о быстром умножении матриц, М. Дж. Фишер и А.Р. Мейер [67] получили булеву схему в базисе $\{\&, \vee,-\}$, которая вычисляет булево произведение любых двух квадратных булевых матриц порядка $n$ и содержит не более $O\left(n^{\log _{2} 7}(\log n)^{1+\varepsilon_{n}}\right)$ элементов, где $\varepsilon_{n} \rightarrow 0$ при $n \rightarrow \infty$.

И. Вегенер [317] рассмотрел следуюшую задачу. Пусть $A_{1}, \ldots, A_{m}$ - совокупность булевых матрищ, каждая из которых имеет размер $n \times k, m \geqslant 2$. С этой совокупностью матриц связьвается такая система $F_{n k}^{m}$, состоящая из $n^{m}$ монотонных булевых функций $\left\{f_{h_{1}, \ldots, h_{m}}\right\}, 1 \leqslant h_{1}, \ldots, h_{m} \leqslant n$, что функция $f_{h_{1}, \ldots, h_{m}}$ равна 1 тогда и только тогда, когда $h_{1}$-я строка матрицы $A_{1}, h_{2}$-я строка матрицы $A_{2}$ и т. д. и, наконец, $h_{m}$-я строка матрицы $A_{m}$ являются такими, что в перечисленных строках имеется по крайней мере одна общая единичная компонента. При $m=2$ система функций из $F_{n k}^{m}$ задает обычное булево произведение матрицы $A_{1}$ и матрицы, транспонированной к матрице $A_{2}$. 
Требуется получить нетривиальную нижнюю оценку сложности минимальной булевой схемы с $n k m$ входами и $n^{m}$ выходами в базисе $\{\&, \vee\}$, которая для любых булевых матрищ $A_{1}, \ldots, A_{m}$, поступаюших на ее вход, вычисляет значение функций из системы $F_{n k}^{m}$.

И. Вегенер в [318] (см. также [317]) доказал, что такая схема содержит $\Omega\left(n^{2} / \log ^{2} n\right)$ элементов. Впоследствии он улучшил эту оценку, доведя ее до величины $\Omega\left(n^{2} / \log n\right)$ (см. [321], [323]).

Некоторые перечисленные результаты освешены в обзорах [30] и [325]. Другие результаты по этой тематике имеются в работах [106], [269], [218], [172], [82], [298], [11], [270], [258] (ссылки приведены в хронологическом порядке).

\section{§ 2.8. Высокие нижние оценки. Методы Разборова и Андреева}

Существенное продвижение в получении высоких нижних оценок для размера минимальных булевых схем в базисе $\{\&, \bigvee\}$, вычисляюших естественные монотонные булевы функции, произошло в 1985 году. А. А. Разборов в [239] и [240] установил сверхполиномиальные нижние оценки (по числу переменных) для размера таких минимальных булевых схем в базисе $\{\&, \vee\}$, которые вычисляют логический перманент квадратных булевых матриц и распознают наличие клики заданного размера в неориентированных графах.

Практически одновременно А. Е. Андреев [9] (см. также [12]) установил квазиэкспоненциальные нижние оценки для размера подобных схем, вычисляюших менее естественные монотонные булевы функции. Эти функции обычно называются функциями Андреева. Хотя полученные А. А. Разборовым и А.Е. Андреевым оценки относятся к булевым схемам, вычисляюшим разные булевы функции, и значительно отличаются друг от друга по величине, методы, с помощью которых получены эти оценки, довольно похожи.

ЗАмЕчАниЕ. Многие авторы статей допускают вольность и оценки вида $\exp \left\{\Omega\left(n^{\alpha}\right)\right\}$, где $n$ - число входов булевой схемы, а константа $\alpha$ удовлетворяет условию $0<\alpha<1$, называют экспоненциальными. Они такими не являются. Мы предлагаем назьвать их квазиэкспоненииальными.

Сначала изложим результаты А. А. Разборова. В случае логического перманента булевых матриц точная постановка такова.

Пусть $A=\left(a_{i, j}\right), 1 \leqslant i, j \leqslant n,-$ произвольная булева матрица размера $n \times n$. Логическим перманентом матрицы $A$ назьвается функция

$$
\operatorname{per} A=\bigvee_{\sigma} \bigwedge_{i=1}^{n} a_{i, \sigma(i)}
$$

где дизъюнкция берется по всем взаимно однозначным отображениям $\sigma:\{1,2, \ldots, n\}$ $\rightarrow\{1,2, \ldots, n\}$.

Пусть $\operatorname{PERM}\left(x_{1,1}, x_{1,2}, \ldots, x_{1, n}, x_{2,1}, x_{2,2}, \ldots, x_{2, n}, \ldots, x_{i, j}, \ldots, x_{n, n}\right)$ обозначает булеву функцию от $n^{2}$ переменных, которая на наборе $\left(a_{1,1}, a_{1,2}, \ldots, a_{1, n}, a_{2,1}, a_{2,2}\right.$, $\left.\ldots, a_{2, n}, \ldots, a_{i, j}, \ldots, a_{n, n}\right)$ равна 1 , если перманент матрицы $A=\left(a_{i, j}\right)$ равен 1, и равна 0 , если per $A=0$. 
Легко видеть, что эта функция является монотонной. Поэтому она может быть вычислена булевой схемой в базисе $\{\&, \vee\}$ с $n^{2}$ входами.

Обозначим через $L^{+}(\operatorname{PERM}(n))$ размер минимальной булевой схемы в базисе $\{\&, \vee\}$, которая вычисляет логический перманент квадратных булевых матриц порядка $n$.

А. А. Разборовьм в [239] сформулирована, а в [240] доказана следующая теорема.

ТЕОРема 2.9. При любом $n \geqslant 1$

$$
L^{+}(\operatorname{PERM}(n)) \geqslant n^{\left(\frac{1}{16}-\varepsilon_{n}\right) \log _{2} n},
$$

əде $\varepsilon_{n} \rightarrow 0$ nрu $n \rightarrow \infty$.

Эта теорема утверждает, что логический перманент булевых матрищ не может быть вычислен булевой схемой в базисе $\{\&, \vee\}$ с полиномиальным числом элементов относительно размера матрищ. Вместе с тем из работы [106] следует, что логический перманент таких матрищ вычислим такой булевой схемой в базисе $\{\&, \vee,-\}$, в которой содержится не более $\Omega\left(n^{5}\right)$ элементов. Таким образом, отношение размера минимальных булевых схем в базисе $\{\&, \vee\}$, вычисляюших логический перманент квадратных булевых матрищ, к размеру аналогичных схем в базисе $\{\&, \vee,-\}$ оказьвается более чем полиномиальным относительно числа переменных.

Известно, что любая квадратная булева матрица $A$ порядка $n$ является матрицей смежности двудольного графа $G$, доли которого содержат по $n$ вершин, а логический перманент матрищы $A$ равен 1 тогда и только тогда, когда в $G$ имеется совершенное паросочетание. Поэтому вычисление перманента квадратных булевых матрищ порядка $n$ и обнаружение совершенного паросочетания в соответствуюших двудольных графах осушествимы одной и той же булевой схемой.

Задача о сложности распознавания наличия клики заданного размера в $m$-вершинных неориентированных граф̆ах состоит в следующем.

Обозначим через $\mathscr{G}(m)$ множество всех неориентированных графов с множеством вершин $V=\left\{v_{1}, \ldots, v_{m}\right\}$. Подмножество $V^{\prime} \subseteq V$ называется кликой в графе $G \in$ $\mathscr{G}(m)$, если любые две вершины из $V^{\prime}$ смежны в графе $G$. Число вершин в клике называется ее размером.

Двоичный набор $\left(a_{1,2}, \ldots, a_{1, m}, a_{2,3}, \ldots, a_{i, j}, \ldots, a_{m-1, m}\right), i<j$, длины $\left(\begin{array}{c}m \\ 2\end{array}\right)$ назовем кодом графа $G \in \mathscr{G}(m)$, если этот набор является таким, что

1) $a_{i, j}=1$, если в $G$ вершины $v_{i}$ и $v_{j}, i<j$, смежны;

2) $a_{i, j}=0$, если в $G$ вершины $v_{i}$ и $v_{j}, i<j$, несмежны.

Обозначим через $\mathrm{CLIQUE}_{s}\left(x_{1,2}, \ldots, x_{1, m}, x_{2,3}, \ldots, x_{i, j}, \ldots, x_{m-1, m}\right), i<j$, такую булеву функцию от $\left(\begin{array}{c}m \\ 2\end{array}\right)$ переменных, что на двоичном наборе $\left(a_{1,2}, \ldots, a_{1, m}\right.$, $\left.a_{2,3}, \ldots, a_{i, j}, \ldots, a_{m-1, m}\right)$ функция равна 1 , если этот набор является кодом графа, содержащим клику размера $s$, и равна 0, если этот набор является кодом графа, в котором нет клики размера $s$.

Легко видеть, что введенная булева функция является монотонной. Поэтому она вычислима булевой схемой в базисе $\{\&, \vee\}$ с $\left(\begin{array}{c}m \\ 2\end{array}\right)$ входами.

Пусть $L^{+}(\operatorname{CLIQUE}(m, s))$ обозначает размер минимальной булевой схемы в базисе $\{\&, \vee\}$, которая вычисляет функцию $\mathrm{CLIQUE}_{s}\left(x_{1,2}, \ldots, x_{1, m}, x_{2,3}, \ldots, x_{i, j}, \ldots\right.$, $\left.x_{m-1, m}\right)$.

А. А. Разборовьм [239] доказана следующая теорема. 
Tеорема 2.10. Ecлu $s=$ const, mo npu $m \rightarrow \infty$

Ecли $s=\Theta(\log m)$, mо при $m \rightarrow \infty$

$$
L^{+}(\operatorname{CLIQUE}(m, s))=\Omega\left(m^{s} /(\log m)^{2 s}\right) .
$$

$$
L^{+}(\operatorname{CLIQUE}(m, s))=m^{\Omega(\log m)} .
$$

За цикл работ этого направления (см. [175]) А. А. Разборов получил премию Рольфа Неванлинны (Rolf Nevanlinna Prize) в 1990 г. на Международном конгрессе математиков в Киото.

При получении результатов из [9] и [12] А. Е. Андреев использовал сходные методы. В [9] он рассмотрел последовательность менее естественных монотонных булевых функций и показал, что минимальные булевы схемы в базисе $\{\&, \vee\}$, которые вычисляют функции из этой последовательности, имеют квазиэкспоненциальный размер относительно числа переменных. Другая последовательность специальных монотонных булевых функций, которые также могут быть вычислены булевыми схемами в базисе $\{\&, \bigvee\}$, содержашими не менее чем квазиэкспоненциальное число элементов (не менее $2^{n^{1 / 3-o(1)}}$ относительно числа переменных $\left.n\right)$, приведена в [12].

Для четкого описания результатов А.Е. Андреева требуются специальные понятия. Мы не будем на этом останавливаться. Отметим лишш, что в работе А. Е. Андреева используются другие понятия, нежели у А. А. Разборова. Для пояснения связи их результатов А. А. Разборов [243; с. 1483-1484] привел перевод понятий А. Е. Андреева на язык своих работ.

Техника доказательств, придуманная А. А. Разборовьм, оказалась весьма эффективной. Она основана на использовании предложенного им в [239] метода аппроксимаций. Этот метод применим во многих случаях, хотя в каждой конкретной ситуации его использование требует отдельных, весьма тонких рассмотрений.

Обший план метода аппроксимаций таков. Пусть требуется получить сверхполиномиальную или квазиэкспоненциальную нижнюю оценку для размера минимальной булевой схемы в базисе $\{\&, \vee\}$, вычисляюшей некоторую монотонную булеву функцию $f$. Для этой цели рассматривается некоторый класс булевых функций, назьваемых "простыми", а также определяется специальное расстояние между двумя булевыми функциями. При этом расстояние функции $f$ до любой простой функции сверхполиномиально или квазиэкспоненщиально велико. Наряду с этим вводятся новые би-

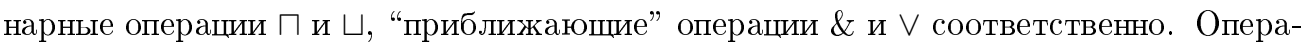
ции $\sqcap$ и $\sqcup$ обладают следуюшими свойствами. Если в схеме $S_{1}$, вычисляющей булеву функцию $f_{1}$, один конъюнктор (или дизъюнктор) заменить на элемент, реализующий операцию $П$ (или $\sqcup)$, то полученная схема будет вычислять такую булеву функцию $f_{2}$, что расстояние между $f_{1}$ и $f_{2}$ не превосходит 1 . Кроме того, после замены в исходной схеме $S$ всех конъюнкторов и дизъюнкторов на элементы $\sqcap$ и $\sqcup$ соответственно получается схема, вычисляющая “простую” булеву функцию. Таким образом, расстояние между этой “простой” функцией и исходной функцией $f$ сверхполиномиально или квазиэкспоненциально велико. Следовательно, исходная схема $S$ имеет сверхполиномиальньй или квазиэкспоненциальный размер.

Усовершенствовав комбинаторную технику доказательства из [240], Н. Алон и Р. Боппана усилили нижнюю оценку А.А. Разборова для размера булевых схем в базисе $\{\&, \vee\}$, которые распознают наличие клик размера $s$ в $m$-вершинных неориентированных графах. В работе [5] ими доказана следуюшая теорема. 
ТЕОРема 2.11. При любом фиксированном $s \geqslant 1 u m \rightarrow \infty$

$$
L^{+}(\operatorname{CLIQUE}(m, s))=\Omega\left(m^{s} /(\log m)^{s}\right) .
$$

Ecли $s=\Theta(\log m)$, mо при $m \rightarrow \infty$

$$
L^{+}(\operatorname{CLIQUE}(m, s))=\exp \left\{\Omega\left(m^{1 / 6} \log ^{1 / 3} m\right)\right\} .
$$

Кроме того, в этой работе улучшена нижняя оценка для размера минимальной булевой схемы в базисе $\{\&, \vee\}$, вычисляюшей функцию из [9], которую часто называют функцией Андреева.

Впоследствии оценки из теоремы 2.11 улучшили К. Амано и А. Маруока [6]. Они доказали следующее утверждение.

ТЕОРема 2.12. При любом фиксированном $s, 5 \leqslant s \leqslant m / 4$, справедливо неравенство

$$
L^{+}(\operatorname{CLIQUE}(m, s)) \geqslant(1.2)(1.8)^{\sqrt{s-1} / 2},
$$

a npu $s=\left\lfloor m^{2 / 3}\right\rfloor u m \rightarrow \infty$

$$
L^{+}(\operatorname{CLIQUE}(m, s))=\exp \left\{\Omega\left((m / \log m)^{1 / 3}\right)\right\} .
$$

Подробное изложение результатов Разборова, Андреева, Алона и Бопшана имеются в работе [57] и монографии [55].

В работах [244], [245], [247], [248], [249] и [250] приведены более простые доказательства утверждений из [239], обсуждена применимость предложенных методов в более обших ситуациях и дан обзор результатов других авторов.

В работе [8] исследовался вопрос о том, как зависит сложность минимальных булевых схем в базисе $\{\&, \vee,-\}$, распознаюших наличие клик заданного размера в $m$-вершинных неориентированных графах от числа используемых инверторов. В ней была доказана следующая теорема.

Теорема 2.13. Если булева схема $S$ в базисе $\{\&, \vee,-\}$ распознает наличие

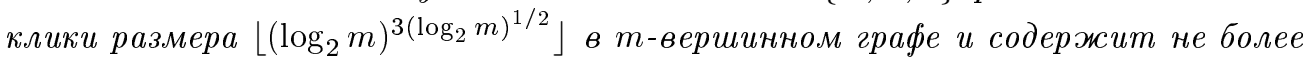
$\left\lfloor(1 / 6) \log _{2} \log _{2} m\right\rfloor$ инверторов, то в $S$ имеется не менее $2^{(1 / 5)\left(\log _{2} m\right)^{\left(\log _{2} m\right)^{1 / 2}}}$ элементов.

Аналогичный вопрос изучался в работах [7], [66] и [281]. К тематике высоких нижних оценок также относятся работы [295], [58], [114], [90], [260], [103], [91] и [98].

\section{§ 2.9. Булевы схемы ограниченной глубины}

В работах по булевьм функщиям утвердилось одно и то же название “глубина" булевых схем для двух разных понятий. В одних случаях под глубиной булевых схем понимается следующий параметр.

Пусть $S$ - произвольная булева схема. Ориентированньй путь от входа к выходу схемы $S$ назьвается иепью. Число вершин в цепи, отличных от входа, называется длиной цепи. Длина максимальной цепи в $S$ назьвается глубиной схемы $S$ и обозначается через $l(S)$. 
Для того чтобы отличать это понятие от глубины булевых схем, используемых в другом смысле, величину $l(S)$ будем называть длиной схемы $S$ (такое название навеяно известным понятием "ширина" булевой схемы).

Часто под глубиной булевых схем понимается другой параметр. Пусть $S$ - произвольная булева схема в базисе $\mathscr{E}$, входами которой являются переменные и их отрицания. Каждой ориентированной цепи $C$, направленной от входа к выходу схемы $S$, ставится в соответствие цеп $C^{\prime}$, получаемая из цепи $C$ удалением входной вершины. Цепь $C^{\prime}$ разбивается на такие подщепи $C_{1}^{\prime}, C_{2}^{\prime}, \ldots, C_{d}^{\prime}$, что

1) каждая вершина цепи $C^{\prime}$ принадлежит только одной подшепи;

2) во всех вершинах одной подщепи реализуется одна и та же функция из $\mathscr{E}$;

3 ) в вершинах подшепи $C_{i}^{\prime}, 1 \leqslant i \leqslant d-1$, реализуется функция, отличная от той, которая реализуется в вершинах подцепи $C_{i+1}^{\prime}$.

Цепь $C^{\prime}$ с максимальньм числом таких подщепей называется максимальной. Число подцепей в максимальной цепи $C^{\prime}$ назьвается глубиной схемы $S$ и обозначается через $D(S)$.

Если булева схема $S$ состоит только из двувходовых дизъюнкторов и конъюнкторов, то ее глубина иногда определяется следуюшим образом. В схеме $S$ каждая неудлиняемая цепь, состоящая только из дизъюнкторов (конъюнкторов), заменяется на один дизъюнктор (конъюнктор) с необходимьм числом входов. В результате получается булева схема $S^{\prime}$, которая реализует ту же функцию, что и схема $S$, а высота схемы $S^{\prime}$ равна глубине схемы $S$.

Пусть

$$
l_{\mathscr{E}}(f)=\min l_{\mathscr{E}}(S),
$$

где минимум берется по всем булевым схемам $S$ без ветвлений в базисе $\mathscr{E}$, вычисляюшим булеву функцию $f$, и

$$
l_{\mathscr{E}}(n)=\max l_{\mathscr{E}}(f),
$$

где максимум берется по всем монотонным булевым функциям от $n$ переменных.

Справедлива следующая теорема.

ТЕОРема 2.14. При любом $n \geqslant 2$

$$
n-\frac{1}{2} \log _{2} n-\log _{2} \log _{2} n+c_{1} \leqslant l_{\{\&, \vee\}}(n) \leqslant n-\frac{1}{2} \log _{2} n-\log _{2} \log _{2} n+c_{2},
$$

где с $c_{1}$ и $c_{2}-$ константы, не зависящие от $n$.

Нижняя оценка для $l_{\{\&, \vee\}}(n)$ следует из (2.7) и того легко проверяемого факта, что если булева схема $S$ с одним выходом в базисе $\{\&, \vee\}$ состоит из $N$ элементов, то $l_{\{\&, \vee\}}(S)>\log _{2} N$. Доказательство верхней оценки для $l_{\{\&, \vee\}}(n)$ имеется в [176].

Пусть $H$ - произвольньй замкнутьй класс монотонных булевых функций, $H(n)-$ множество функций из $H$, зависящих от $n$ переменных, $\mathscr{E}-$ произвольный конечньй базис для функций из $H$ и

$$
l_{\mathscr{E}}(H(n))=\max l_{\mathscr{E}}(f),
$$

где максимум берется по всем функциям $f$ из $H(n)$. 
А. Б. Угольников [350] получил полное (с точностью до порядка) описание поведения функщии $l_{\mathscr{E}}(H(n))$ для каждого замкнутого класса $H$ монотонных булевых функций и любого конечного базиса $\mathscr{E}$ для функщий из $H$.

Приведем ряд результатов, относяшихся к размеру и длине булевых схем в базисе $\{\&, \vee\}$, вычисляющих конкретные монотонные булевы функции.

А. Яо в работе [343] доказал, что любая булева схема в базисе $\{\&, \vee\}$, которая распознает связность неориентированных $n$-вершинных графов, имеет длину, равную $\Omega\left((\log n)^{3 / 2} / \log \log n\right)$.

В [80] и [81] доказаны следуюшие утверждения.

ТЕОРема 2.15. 1. Пусть булева схема $S$ в базисе $\{\&, \vee\}$ имеет размер $n^{d}$ и распознает связность неориентированных $n$-вериинных графов. Если $d=$ $o\left((\log n)^{1 / 2}\right)$, то длина схемь $S$ равна $\Omega\left((\log n)^{2} / \log d\right)$.

2. Если булева схема $S$ без ветвлений в базисе $\{\&, \vee\}$ распознает связность неориентированньх п-вершинных графов, то длина схемы $S$ равна $\Omega\left((\log n)^{2} / \log \log n\right)$.

Подобные булевы схемы изучались также в работах [116], [117].

В работах [237] и [238] рассмотрены такие булевы схемы в базисе $\{\&, \bigvee\}$, которые распознают наличие паросочетаний с заданным числом ребер в неориентированных графах. В этих работах доказана следующая теорема.

Теорема 2.16. Пусть $m$ - произвольное натуральное число и $n=3 \mathrm{~m}$. Тогда длина любой булевой схемы в базисе $\{\&, \vee\}$, которая распознает наличие паросочетаний с т ребрами в неориентированных $n$-вершинных графах, равна $\Theta(n)$.

Перейдем к изложению результатов, относяшихся к размеру булевых схем фиксированной глубины, вычисляющих индивидуальные монотонные булевы функции.

В 1970 г. О.Б. Лупанов [185] показал, что максимальные (по числу элементов) булевы схемы без ветвлений, которые имеют разную глубину и вычисляют одну и ту же монотонную булеву функцию, могут иметь существенно разньй размер. В качестве таких функций он рассмотрел последовательность монотонных булевых функций

$$
f_{n}\left(x_{1}, \ldots, x_{n} ; y_{1}, \ldots, y_{n}\right)=\bigvee_{i=1}^{n} x_{i} y_{i} y_{i+1} \cdots y_{n}
$$

$n=1,2, \ldots$, и доказал следуюшее утверждение.

Пусть $L_{\&}^{d}\left(f_{n}\right)\left(L_{\vee}^{d}\left(f_{n}\right)\right)$ обозначает число элементов в минимальной булевой схеме без ветвлений в базисе $\{\&, \vee\}$, которая имеет глубину $d$ и вычисляет функцию $f_{n}$ из (2.17), а ее выходной элемент является конъюнктором (дизъюнктором).

ТЕОРема 2.17. При любом фиксированном $d \geqslant 2 u n \rightarrow \infty$

$$
L_{\&}^{d}\left(f_{n}\right) \sim L_{\vee}^{d}\left(f_{n}\right) \sim \frac{n(d-1)}{d}((d-1) ! n)^{1 /(d-1)} .
$$

Позднее размер булевых схем без ветвлений заданной глубины, вычисляющих функции $f_{n}$ из $(2.17)$, исследовался в работах [42]-[44]. В них были получены следуюшие результаты. 
В [43] показано (набросок доказательства имеется в [42]), что функция $f_{n}$ может быть вычислена такой булевой схемой $S$ без ветвлений в базисе $\{\&, \vee\}$, что произведение числа элементов в $S$ и ее глубины равно $\Omega\left(n \log ^{2} n\right)$. (Подробное доказательство этого утверждения имеется в монографии [55].) Кроме того, доказано, что среди таких схем имеется схема глубины $\Theta(\log n)$.

В [44] доказано, что функция $f_{n}$ из $(2.17)$ может быть вычислена такой булевой схемой $S$ без ветвлений в базисе $\{\&, \vee\}$, что произведение числа элементов в $S$ и ее глубины по порядку равно $\frac{n \log n \log \log \log n}{\log \log \log \log n}$.

Симметрическая монотонная булева функция $f\left(x_{1}, \ldots, x_{n}\right)$ называется функцией голосования, если при любом наборе $\left(\sigma_{1}, \ldots, \sigma_{n}\right) \in E^{n}$

$$
f\left(\sigma_{1}, \ldots, \sigma_{n}\right)= \begin{cases}0, & \text { если } \sigma_{1}+\cdots+\sigma_{n}<\lceil n / 2\rceil, \\ 1 & \text { в противном случае. }\end{cases}
$$

Функция голосования от $n$ переменных часто обозначается через МАЈ $(n)$.

Обозначим через $L_{\mathscr{E}}^{d}(\mathrm{MAJ}(n))$ число элементов в минимальной булевой схеме без ветвлений глубины $d$ в базисе $\mathscr{E}$, которая вычисляет функцию $\mathrm{MAJ}(n)$.

Р. Боппана исследовал поведение функции $L_{\{\&, \vee\}}^{d}(\mathrm{MAJ}(n))$. В работе [28] (см. также [26]) им доказана

ТЕорема 2.18. При любом фиксированном $d$ и произвольном $n$

$$
L_{\{\&, \vee\}}^{d}(\operatorname{MAJ}(n))=\exp \left\{\Omega\left(n^{1 /(d-1)}\right)\right\} .
$$

Аналогичный результат получил Дж. Хостад (см. [100], [101]).

Вместе с тем Л. Вальянт [308] доказал, что при каждом четном $n$ существует булева схема без ветвлений в базисе $\{\&, \bigvee\}$, которая вычисляет функцию $\mathrm{MAJ}(n)$ и имеет размер, по порядку не превосходяший $n^{5.3}$. Доказательство этого утверждения имеется также в монографиии [55].

А. А. Разборов изучал поведение функции $L_{\mathscr{E}}^{d}(\mathrm{MAJ}(n))$ в случаях, когда $\mathscr{E}=\{\&, \oplus\}$ и $\mathscr{E}=\{\&, \vee, \oplus\}$. В работе [242] им доказана следуюшая теорема.

ТЕОРема 2.19. При любом фиксированном $d$ и произвольном $n$

$$
\begin{aligned}
L_{\{\&, \oplus\}}^{d}(\operatorname{MAJ}(n)) & =\exp \left\{\Omega\left(n^{1 /(d+1)}\right)\right\}, \\
L_{\{\&, \vee, \oplus\}}^{d}(\operatorname{MAJ}(n)) & =\exp \left\{\Omega\left(n^{1 /(2 d+2)}\right)\right\} .
\end{aligned}
$$

Соотношение (2.18) и набросок его доказательства имеются в [241]. Другое доказательство приведено в монографии [55].

Обобщение утверждения теоремы 2.19 имеется в работе [282].

Булева схема глубины 3 без ветвлений в базисе $\{\&, \vee\}$, в которой входные полюсы помечены переменньми и их отрицаниями, называется $\Sigma \Pi \Sigma$-схемой, если выходным элементом этой схемы является дизъюнктор. Если выходным элементом такой схемы является конъюнктор, то схема назьвается П $\Sigma$-схемой.

Пусть $\Sigma \Pi \Sigma$-схема (П $\Sigma$ П-схема) имеет $2\left(\begin{array}{c}n \\ 2\end{array}\right)$ входов, которые помечены переменными $x_{1,2}, x_{1,3}, \ldots, x_{1, n}, x_{2,3}, \ldots, x_{i, j}, \ldots, x_{n-1, n}$ и их отрицаниями, и вычисляет функцию $\operatorname{CLIQUE}_{s}\left(x_{1,2}, x_{1,3}, \ldots, x_{1, n}, x_{2,3}, \ldots, x_{i, j}, \ldots, x_{n-1, n}\right)$, определенную в $\S 2.8$.

Л. Вальянт в [307] доказал следуюшее утверждение. 
ТеОРема 2.20. Пусть $m$ - произвольное натуральное число, $n=7 m^{4} u$

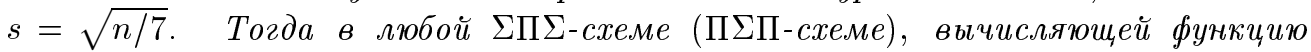
$\mathrm{CLIQUE}_{s}\left(x_{1,2}, x_{1,3}, \ldots, x_{1, n}, x_{2,3}, \ldots, x_{i, j}, \ldots, x_{n-1, n}\right)$, содержится не менее $c^{n^{1 / 4}}$ әлементов, где $c$-некоторая константа, $0<c<2$.

В работах [231] и [232] изучался размер $\Sigma \Pi \Sigma$-схем и П $\Sigma \Pi$-схем, вычисляющих симметрические монотонные функции $f_{r}\left(x_{1}, \ldots, x_{n}\right)$ (функция $f_{r}\left(x_{1}, \ldots, x_{n}\right.$ ) равна 1 на любом наборе значений переменных $x_{1}, \ldots, x_{n}$, содержашем не менее $r$ единиц, и равна 0 на остальных наборах этих переменных). Такие функции часто называются $n о-$ роговыми функциями с порогом $r$.

В этих работах доказаны следуюшие утверждения.

ТЕОРема 2.21 [231]. При $n \rightarrow \infty$ любом $r, 2 \leqslant r \leqslant n / 2$, в минималь-

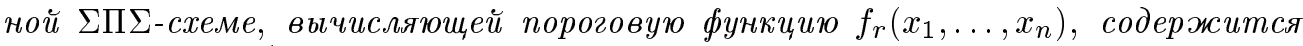
$\exp \left\{\Theta\left((r / \log r)^{1 / 2}\right)\right\} n \log n$ әлементов.

ТЕОРема 2.22 [232]. При любом $r, 4 \leqslant r \leqslant \log _{2} n$, в минимальной ПгП-схеме, вычисляющей пороговую функцию $f_{r}\left(x_{1}, \ldots, x_{n}\right)$, содержится не менее $\exp \{\Theta(r)\} n^{2}$ и не более $e^{r} n^{2} \log _{2} n$ әлементов. При $r=2 u r=3$ в таких ПгП-схемах число элементов по порядку равно $n^{3 / 2}$ и $n^{2}$ әлементов соответственно.

Другие результаты по тематике, освешенной в этом параграфе, содержатся в работаx [193], [324], [135], [2], [282], [115], [79], [111], [112], [236].

Результаты по близкой тематике имеются в работах [72], [322], [1], [73], [341], [60], [45], [246], [330].

В обоих случаях ссылки приведены в хронологическом порядке.

\section{§ 2.10. Дизъюнктивные нормальные формы}

Литералом назьвается булева переменная или ее отрицание.

Элементарной конбюнкиией называется логическое произведение различных литералов, в которых отсутствуют литералы вида $x$ и $\bar{x}$.

Дизбюнктивной нормальной формой (д.н.ф.) назьвается дизъюнкция различных элементарных конъюнкций. Каждую д.н.ф. можно рассматривать как булеву функцию в базисе $\{\&, \vee,-\}$.

Д.н.ф. $D$, задаюшая (реализуюшая) булеву функцию $f$, назьвается кратчайшей, если в $D$ содержится наименьшее число конъюнкций среди всех д.н.ф., задающих $f$.

Д.н.ф. $D$, задаюшая булеву функцию $f$, назьвается минимальной, если $D$ содержит наименьшее число литералов среди всех д.н.ф., задающих $f$.

Д.н.ф. $D$, задаюшая булеву функцию $f$, назьвается сокращенной, если $D$ состоит из всех таких простых конъюнкций, что после вычеркивания хотя бы одного литерала из некоторой конъюнкции получается д.н.ф., которая задает булеву функцию, отличную от $f$.

Д.н.ф. $D$, задаюшая булеву функцию $f$, называется тупиковой, если $D$ состоит из всех таких простых конъюнкций, что после вьчеркивания хотя бы одного литерала из некоторой конъюнкции или удаления какой-либо конъюнкции из $D$ получается д.н.ф., которая задает булеву функцию, отличную от $f$. 
Для дизъюнктивных нормальных форм известны следуюшие факты:

1) в кратчайших д.н.ф., задаюших булевы функции

$$
\begin{aligned}
& f^{1}\left(x_{1}, \ldots, x_{n}\right)=x_{1}+\cdots+x_{n}(\bmod 2), \\
& f^{2}\left(x_{1}, \ldots, x_{n}\right)=x_{1}+\cdots+x_{n}+1(\bmod 2),
\end{aligned}
$$

содержится по $2^{n-1}$ конъюнкций [181];

2) в каждой тупиковой д.н.ф., задающей произвольную булеву функцию от $n$ переменных, содержится не более $2^{n-1}$ конъюнкций [181];

3) почти каждая булева функция от $n$ переменных может быть задана очень большим числом тупиковых д.н.ф.;

4) число конъюнкций в кратчайшей д.н.ф., задающей почти каждую булеву функцию от $n$ переменных, не превосходит $c \cdot 2^{n} /\left(\log _{2} n \cdot \log _{2} \log _{2} n\right)$, где $c<5$ подходящая константа [153].

Для функщий из $M(n)$ ситуашия существенно иная. Во-первых, сокращенная д.н.ф., задающая произвольную монотонную булеву функцию, одновременно является тупиковой, кратчайшей и минимальной д.н.ф. функции $f$. Во-вторых, для сокрашенных д.н.ф., задаюших монотонные булевы функции, справедливы следуюшие утверждения.

1. Б. С. Кочкарев [140] (см. также [292]) доказал, что при любом $n$ в сокрашенной д.н.ф. любой функции из $M(n)$ содержится не более $\lceil n / 2\rceil\left(\begin{array}{c}n \\ \lfloor n / 2\rfloor\end{array}\right)$ литералов.

2. Согласно теореме 2.11 в сокращенной д.н.ф. любой функции из $M(n)$ имеется не более $\left(\begin{array}{c}n \\ \lfloor n / 2\rfloor\end{array}\right)$ конъюнкций; при каждом четном $n$ имеется только одна функция из $M(n)$ такая, что в ее сокращенной д.н.ф. содержится $\left(\begin{array}{c}n \\ n / 2\end{array}\right)$ конъюнкций, а при каждом нечетном $n$ - две функции из $M(n)$.

3. Из теорем 1.3 и 1.4 следует, что почти каждая функция из $M(n)$ является такой, что в ее сокращенной д.н.ф. имеется $\frac{1}{2}\left(\begin{array}{c}n \\ \lfloor n / 2\rfloor\end{array}\right)(1+\varepsilon(n))$ конъюнкций и $\frac{1}{4} n\left(\begin{array}{c}n \\ \lfloor n / 2\rfloor\end{array}\right)(1+$ $\left.\varepsilon_{1}(n)\right)$ литералов, где $\varepsilon(n) \rightarrow 0$ и $\varepsilon_{1}(n) \rightarrow 0$ при $n \rightarrow \infty$.

4. Из теоремы 1.3 следует, что почти каждая функция из $F_{7}^{\infty}(n)$ является такой, что в ее сокрашенной д.н.ф. имеется $\frac{1}{2}\left(\begin{array}{c}n-1 \\ \lfloor(n-1) / 2\rfloor\end{array}\right)\left(1+\varepsilon_{2}(n)\right)$ конъюнкций и $\frac{1}{4} n\left(\begin{array}{c}n-1 \\ \lfloor(n-1) / 2\rfloor\end{array}\right)\left(1+\varepsilon_{3}(n)\right)$ литералов, где $\varepsilon_{2}(n) \rightarrow 0$ и $\varepsilon_{3}(n) \rightarrow 0$ при $n \rightarrow \infty$.

5 . Пользуясь информацией о структуре типичных функций из $F_{7}^{2}(n)$ (см. $\left.\S 1.5\right)$, нетрудно видеть, что почти каждая функция из $F_{7}^{2}(n)$ является такой, что в ее сокращенной д.н.ф. содержится $\frac{1}{2}\left(\begin{array}{c}n \\ \lfloor n / 2\rfloor\end{array}\right)\left(1+\varepsilon_{4}(n)\right)$ конъюнкций и $\frac{1}{4} n\left(\begin{array}{c}n \\ \lfloor n / 2\rfloor\end{array}\right)\left(1+\varepsilon_{5}(n)\right)$ литералов, где $\varepsilon_{4}(n) \rightarrow 0$ и $\varepsilon_{5}(n) \rightarrow 0$ при $n \rightarrow \infty$.

\section{$\S$ 2.11. Лоскутки}

В работе [194] исследовалась возможность вычисления функций $f_{r}\left(x_{1}, \ldots, x_{n}\right)$, $1 \leqslant r \leqslant n$, так назьваемыми плоскими булевыми схемами в базисе $\{\&, \vee\}$. (Определение таких схем имеется, например, в [55].) В ней доказано, что при любом $n \geqslant 5$ только функции $f_{1}\left(x_{1}, \ldots, x_{n}\right), f_{2}\left(x_{1}, \ldots, x_{n}\right), f_{n-2}\left(x_{1}, \ldots, x_{n}\right), f_{n-1}\left(x_{1}, \ldots, x_{n}\right)$ и $f_{n}\left(x_{1}, \ldots, x_{n}\right)$ могут быть вычислены такими схемами.

В работе [21] выяснено, какие произвольные монотонные булевы функции могут быть вычислены плоскими булевыми схемами. К этому же направлению относится работа [82]. 
А. А. Вороненко [315] рассматривал следующую задачу распознавания монотонности произвольных булевых функщий.

Имеется булева схема $S$ с $2^{n}$ входами в произвольном конечном базисе $\mathscr{E}$, которая работает следуюшим образом. Если на входы схемы $S$ подается двоичный набор длины $2^{n}$, рассматриваемый как столбец значений булевой функции $f$, заданной таблично, то на выходе схемы $S$ появляется 1 , если $f$ - монотонная функция, и 0 - в противном случае. Спрашивается, какое минимально возможное число элементов может иметь схема $S$ в базисе $\mathscr{E}$. Частичным ответом на этот вопрос является следующий результат из [315]: существует такая булева схема в базисе $\mathscr{E}$ размера не более $O\left(2^{n} \sqrt{n} \log n\right)$, которая распознает свойство монотонности булевых функций от $n$ переменных.

В работе [77] установлена эквивалентность проблемы распознавания самодвойственности монотонных булевых функций и проблемы выполнимости специального вида. Сложность распознавания самодвойственности монотонных булевых функций изучалась в работе [69].

Р. Л. Ривест [256] показал, что монотонные последовательные машины могут быть более экономными, нежели монотонные булевы схемы.

Пусть $f\left(x_{1}, \ldots, x_{n}\right)$ - произвольная булева функция. Функция $g_{k}\left(x_{1}, \ldots, x_{n}\right)$, $0 \leqslant k \leqslant n$, называется $k$-м срезом функции $f\left(x_{1}, \ldots, x_{n}\right)$, если

$$
g_{k}\left(\sigma_{1}, \ldots, \sigma_{n}\right)= \begin{cases}f\left(\sigma_{1}, \ldots, \sigma_{n}\right) & \text { на любом таком наборе }\left(\sigma_{1}, \ldots, \sigma_{n}\right) \in E^{n}, \\ & \text { что } \sigma_{1}+\cdots+\sigma_{n}=k, \\ 0, & \text { если } \sigma_{1}+\cdots+\sigma_{n}<k, \\ 1, & \text { если } \sigma_{1}+\cdots+\sigma_{n}>k .\end{cases}
$$

Функции среза изучались в работах [326], [328], [309]. Другие задачи о монотонных булевых функциях рассматривались в работах [76], [271], [18], [19], [327], [33], [56], [342], [20], [85], [253], [59], [235], [89], [236] (работы перечислены в хронологическом порядке).

\section{Глава 3. Прикладные задачи}

\section{§3.1. Расшифровка монотонных булевых функций}

Задача расшифровки (восстановления) булевых функций заключается в следующем. Пусть заданы некоторое множество $R(n)$ булевых функций от $n$ переменных, и пусть имеется оракул, которьй отвечает на вопрос: чему равно значение неизвестной функции $f \in R(n)$ на предъявленном наборе из $E^{n}$.

Ясно, что если $R(n)$ состоит из всех булевых функций от $n$ переменных, то при расшифровке неизвестной функции из $R(n)$ требуется $2^{n}$ обрашений к оракулу, ибо надо узнать значения функции на всех наборах из $E^{n}$.

Если же множеству $R(n)$ принадлежат не все булевы функции от $n$ переменных, то для восстановления функции с помошњю оракула нужно выяснить значения функции не на всех наборах из $E^{n}$, а на остальных значение функции можно вычислять без обращения к оракулу. 
Например, для расшифровки любой симметрической булевой функции от $n$ переменных достаточно определить ее значения на любых наборах $\widetilde{\alpha}_{0}, \widetilde{\alpha}_{1}, \ldots, \widetilde{\alpha}_{n}$ из $E^{n}$ таких, что в $\widetilde{\alpha}_{i}$ имеется $i$ единиц, $0 \leqslant i \leqslant n$.

Теперь перейдем к задачам расшифровки монотонных булевых функций. Решение этой задачи позволяет сократить перебор объектов в некоторых прикладных задачах таких, как некоторые задачи целочисленного программирования с булевыми переменными [148], выделение всех существенных или тупиковых совокупностей переменных частичных булевых функций [346], построение матрицы оптимального алфавитного кодирования [192], задач распознавания образов [48], [297], [347], выбор алгоритма планирования экспериментов и др.

Сформулируем результаты, относяшиеся к сложности расшифровки функций из $M(n)$. Пусть $f$ - произвольная функция из $M(n)$. При ее расшифровке после очередного обращения к оракулу относительно значения функции $f$ на наборе $\widetilde{\alpha}$ становятся известными не только значение $f(\widetilde{\alpha})$, но и значения на некоторых других наборах. Действительно, если $f(\widetilde{\alpha})=1$, то $f(\widetilde{\beta})=1$ на любом наборе $\widetilde{\beta} \succ \widetilde{\alpha}$; если же $f(\widetilde{\alpha})=0$, то $f(\widetilde{\beta})=0$ на любом наборе $\widetilde{\beta} \prec \widetilde{\alpha}$.

Алгоритмы расшифровки монотонных булевых функций в основном различаются лишь порядком отбора тех наборов, которые предъявляются оракулу (при различных порядках множества вершин, предъявленных оракулу, могут различаться). Пусть $A-$ произвольный алгоритм такого вида. Обозначим через $\varphi_{A}(n)$ минимальное число обрашений алгоритма $A$ к оракулу, достаточное для расшифровки любой функции из $M(n)$ с использованием алгоритма $A$. Далее, пусть $\varphi(n)=\max \varphi_{A}(n)$, где максимум берется по всем алгоритмам $A$ рассматриваемого вида.

Первые исследования поведения функции $\varphi(n)$ были проведены В. К. Коробковым [144], [146]. Он доказал, что

$$
\left(\begin{array}{c}
n \\
n / 2
\end{array}\right)+\left(\begin{array}{c}
n \\
\lfloor n / 2\rfloor+1
\end{array}\right) \leqslant \varphi(n) \leqslant 5\left(\begin{array}{c}
n \\
n / 2
\end{array}\right) .
$$

Минимальное число обращений к оракулу, равное $\left(\begin{array}{c}n \\ n / 2\end{array}\right)+\left(\begin{array}{c}n \\ \lfloor n / 2\rfloor+1\end{array}\right)$, требуется для расшифровки функции $f\left(x_{1}, \ldots, x_{n}\right)$ такой, что

$$
f\left(\alpha_{1}, \ldots, \alpha_{n}\right)=\left\{\begin{array}{l}
1, \text { если }\lfloor n / 2\rfloor<\sum_{i=1}^{n} \alpha_{i} \leqslant n, \\
0, \text { если } 0 \leqslant \sum_{i=1}^{n} \alpha_{i} \leqslant\lfloor n / 2\rfloor .
\end{array}\right.
$$

Верхняя оценка для $\varphi(n)$ получена конструктивно: был предложен такой алгоритм $A$ расшифровки функций из $M(n)$, что $\varphi(n) \leqslant 5\left(\begin{array}{c}n \\ n / 2\end{array}\right)$.

Окончательное решение этой задачи было получено Ж. Анселем [96]. Им доказана следующая теорема.

ТЕОРемА 3.1. При любом $n \geqslant 1$

$$
\varphi(n)=\left(\begin{array}{c}
n \\
n / 2
\end{array}\right)+\left(\begin{array}{c}
n \\
\lfloor n / 2\rfloor+1
\end{array}\right)
$$


Доказательство этой теоремы основано на разбиении множества всех наборов из $E^{n}$ на попарно непересекающиеся специальные цепи.

При реализации как алгоритма Коробкова, так и алгоритма Анселя требуется большой объем памяти. Так, описанный Анселем алгоритм расшифровки функций из $M(n)$ предполагает наличие в памяти некоторой конструкции, содержащей все двоичные слова длины $n$.

Впоследствии были предложены вариации этих алгоритмов [22], [299], [284] с использованием меньшего объема.

Н. А. Соколов [286] описал алгоритм расшифровки монотонных булевых функций, основанный на идеях дихотомии и рекурсии. При расшифровке любой функции из $M(n)$ этот алгоритм обрашается к оракулу не более $\left(\begin{array}{c}n \\ n / 2\end{array}\right)+\left(\begin{array}{c}n \\ \lfloor n / 2\rfloor+1\end{array}\right)$ раз.

Д.Н. Гайнанов [75] изучал расшифровку монотонных булевых функций с другим критерием оптимальности алгоритма.

В [149] рассматривалась расшифровка функций из $M(n)$ посредством локальных алгоритмов, введенных Ю.И. Журавлевьм. Локальность алгоритма означает, что поиск очередного набора из $E^{n}$, предъявляемого оракулу, производится “вблизи” множества тех наборов, на которых значения функции уже определены.

Другие вопросы расшифровки функций из $M(n)$ и аналогичных классов рассматривались в работах [31], [32], [164], [188], [189], [284], [287], [288], [300], [301]. Частичная расшифровка таких функций изучалась в [285].

В.К. Коробков [147] рассматривал расшифровку монотонных функций, заданных на произвольном конечном частично упорядоченном множестве и принимаюших два значения.

Задача о расшифровке монотонных булевых функщий обобщалась на монотонные функции многозначной логики (см., например, [4], [150], [272], [273]).

К расшифровке монотонных булевых функций сводятся некоторые задачи целочисленного линейного программирования. Например, пусть задана система ограничений

$$
\sum_{j=1}^{n} a_{i j} x_{j} \leqslant b_{i}, \quad i=1,2, \ldots, m,
$$

где все $a_{i j} \geqslant 0$, а переменные могут принимать значения 0 и 1 . Требуется найти решение системы (3.1), максимизирующее функционал

$$
F=\sum_{i=1}^{n} c_{i} x_{i}
$$

где все $c_{i} \geqslant 0$.

Задача (3.1), (3.2) часто называется $n$-мерной задачей о рюкзаке (ранще).

В. К. Коробков [148] доказал, что решение этой задачи может быть сведено к задаче построения таблицы значений подходящей функции $g\left(x_{1}, \ldots, x_{n}\right)$ из $M(n)$ и для любой функции из $M(n)$ можно указать соответствующую ей $n$-мерную задачу о рюкзаке.

Так как областью допустимых значений переменных функции $g\left(x_{1}, \ldots, x_{n}\right)$ является множество наборов из $E^{n}$, в которых функция $g\left(x_{1}, \ldots, x_{n}\right)$ равна нулю, а функционал $F$ не убывает по каждой переменной, то эта задача может быть решена путем просмотра всех максимальных допустимых решений - верхних нулей соответствующей функции из $M(n)$. 


\section{§3.2. Максимальные верхние нули монотонных булевых функций}

Пусть $f\left(x_{1}, \ldots, x_{n}\right)$ - произвольная функция из $M(n)$. Верхний нуль $\widetilde{\alpha}=\left(\alpha_{1}, \ldots\right.$, $\left.\alpha_{n}\right)$ функции $f$ из $M(n)$ называется максимальныцм. если для любого верхнего нуля $\widetilde{\beta}=\left(\beta_{1}, \ldots, \beta_{n}\right)$ функции $f$ вьполняется неравенство $\sum_{i=1}^{n} \beta_{i} \leqslant \sum_{i=1}^{n} \alpha_{i}$.

Задача поиска максимального верхнего нуля монотонной булевой функции при условии, что функция неизвестна исследователю, была поставлена Ю. И. Журавлевым. Эта задача представляет большой интерес в связи с тем, что к ней сводятся некоторые дискретные экстремальные задачи, в которых требуется найти глобальньй максимум. Например, к ней сводятся некоторые задачи целочисленного программирования с булевыми переменными, задача нахождения минимальных тестов для бинарных таблиц и т. д. Некоторые из этих задач будут рассмотрены в $\S \S 3.3$ и 3.4.

Задача о поиске максимального верхнего нуля (хотя бы одного, если их несколько) состоит в следующем. Пусть задано некоторое множество $R(n)$ функций из $M(n)$, и пусть имеется оракул, которьй отвечает на вопрос: чему равно значение неизвестной функции $f \in R(n)$ на предъявленном наборе из $E^{n}$ ? Требуется определить, какое число обрашений к оракулу достаточно, чтобы найти максимальньй верхний нуль.

В $\S 3.1$ пояснялось, что после очередного обрашения алгоритма к оракулу относительно значения функции $f$ на предъявленном наборе становятся известными значения функции $f$ и на некоторых других наборах из $E^{n}$. Поэтому число обрашений алгоритма к оракулу менњше $2^{n}$.

Рассмотренные алгоритмы поиска максимальных верхних нулей монотонных булевых функций различаются лишь порядком отбора тех наборов, которые предъявляются оракулу.

Пусть $A$-произвольный алгоритм такого вида. Обозначим через $\mu_{A}(n)$ минимальное число обрашений алгоритма $A$ к оракулу, досточное для нахождения максимального верхнего нуля любой функции из $M(n)$ с использованием алгоритма $A$. Далее, пусть $\mu(n)=\min \mu_{A}(n)$, где минимум берется по всем алгоритмам $A$ такого вида.

Исследование поведения функции $\mu(n)$ было проведено Н.Н. Катериночкиной. В [123] она доказала следующее утверждение.

Tеорема 3.2. При любом $n \geqslant 1$

$$
\mu(n)=\left(\begin{array}{c}
n \\
n / 2
\end{array}\right)+1
$$

Неравенство $\mu(n) \geqslant\left(\begin{array}{c}n \\ n / 2\end{array}\right)+1$ следует из того, что при поиске максимального верхнего нуля функщии $f\left(x_{1}, \ldots, x_{n}\right)$ из $M(n)$ такой, что

$$
f\left(\alpha_{1}, \ldots, \alpha_{n}\right)=\left\{\begin{array}{l}
1, \quad \text { если }\lfloor n / 2\rfloor \leqslant \sum_{i=1}^{n} \alpha_{i} \leqslant n, \\
0 \text { в противном случае, }
\end{array}\right.
$$

число обрашений к оракулу любого алгоритма такого вида не может быть меньше $\mu(n) \geqslant\left(\begin{array}{c}n \\ n / 2\end{array}\right)+1$. Вместе с тем в [123] предложен такой алгоритм $A$ поиска максимального верхнего нуля произвольной функции из $M(n)$, что $\mu(n) \leqslant\left(\begin{array}{c}n \\ n / 2\end{array}\right)+1$.

Ю. И. Журавлев, С. Н. Мирошник и С. М. Швартин [347] описали алгоритм нахождения максимального верхнего нуля, основанньй на идее градиентной оптимизации. 
А. А. Сапоженко исследовал минимальное число обращений к оракулу при поиске максимальных верхних нулей типичных функций из $M(n)$. В [263] он показал, что число таких обращений не превосходит $w(n) 2^{n / 2}$, где $w(n)$ - произвольная функция такая, что $w(n) \rightarrow \infty$ при $n \rightarrow \infty$. Эта величина значительно меньше величины $\mu(n)$.

Н.Н. Катериночкина [128] исследовала задачу поиска максимальных верхних нулей самодвойственных функций из $M(n)$, а также функций из замкнутых классов $F_{7}^{\infty}$ и $F_{7}^{m}, m=2,3, \ldots$ (определение этих классов дано в $\S 1.4$ ). Для большинства классов ей удалось найти нижние и верхние оценки минимального числа обращений к оракулу (эти оценки отличаются друг от друга незначительно). Для остальных классов получены точные значения для таких величин.

В работах [125], [127] (см. также [126]) исследовалась задача поиска максимальных верхних нулей функций из $R(n)$, когда $R(n)$ не совпадает с $M(n)$ и состоит из просто описьваемых функций. В работах $[124],[283]$ использованньй подход применялся для поиска максимальных верхних нулей функций многозначной логики.

\section{§3.3. Пороговые монотонные булевы функции и системы линейных неравенств}

Булева функция $f\left(x_{1}, \ldots, x_{n}\right)$ назьвается пороговой, если существуют действительные числа $w_{1}, \ldots, w_{n}, h$ такие, что

$$
\left\{f\left(x_{1}, \ldots, x_{n}\right)=1\right\} \Leftrightarrow\left\{\sum_{i=1}^{n} w_{i} x_{i} \geqslant h\right\} .
$$

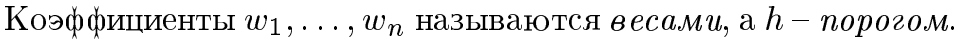

Небольшим изменением весов и порога всегда можно добиться того, чтобы в $E^{n}$ не оказалось набора $\left(\alpha_{1}, \ldots, \alpha_{n}\right)$ такого, что $w_{1} \alpha_{1}+\cdots+w_{n} \alpha_{n}=h$. Аналогично значения весов можно брать рациональными, а стало быть, и целыми.

Пороговым булевым функциям посвящена обширная литература. В обзоре Ю. А. Зуева [351] дано освещение исторического пути развития пороговой логики и описание ее применений. Интерес к пороговьм булевым функциям стимулируется важностью решаемых их с помошью задач. Например, пороговые булевы функции используются в булевом линейном программировании, при диагностике дискретных устройств, вычисляюших булевы функции, при решении задач искусственного интеллекта и распознавании образов и в других разделах прикладной математики.

Если пороговая булева функция $f\left(x_{1}, \ldots, x_{n}\right)$ задается неравенством $w_{1} x_{1}+\cdots+$ $w_{n} x_{n} \geqslant h$, в котором коэффициенты и порог неотрицательны, то $f\left(x_{1}, \ldots, x_{n}\right)$ является монотонной функцией.

Приведем два примера применения монотонных пороговых булевых функций.

ПримеР 1. Симметрические монотонные булевы функции, которые рассматривались в $\S 2.5$, являются пороговыми функциями. Каждая функция $f_{r}\left(x_{1}, \ldots, x_{n}\right)$, $1 \leqslant r \leqslant n$, задается неравенством $x_{1}+\cdots+x_{n} \geqslant r$.

Например, на языке таких функций описьваются результаты голосований. Действительно, пусть голосуют $n$ участников и решение принимается только в том случае, когда не менее половины участников проголосуют положительно. Положив

$$
x_{i}= \begin{cases}1, & \text { если } i \text {-й участник голосует положительно, } \\ 0 & \text { в противном случае, }\end{cases}
$$


получаем, что решение принимается при таких исходах голосования, когда

$$
f_{\lceil n / 2\rceil}\left(x_{1}, \ldots, x_{n}\right)=1
$$

Если решение принимается только в тех случаях, когда не менее $2 / 3$ участников проголосует положительно, то исход голосования описывается функцией $f_{\lceil 2 n / 3\rceil}\left(x_{1}, \ldots, x_{n}\right)=1$.

Иногда проводится взвешенное голосование, т.е. такое, когда каждому участнику приписывается положительньй вес (согласно его степени компетентности). В таком случае исход голосования описывается пороговой монотонной функцией более общего вида.

ПримеР 2. При построении дискретных устройств, вычисляюших булевы функции, для повьшения надежности их функционирования применяется избыточность. Нередко в таких случаях используются мажоритарные элементы, которые реализуют функцию $f_{r}\left(x_{1}, \ldots, x_{n}\right)$.

Пусть $T(n)$ обозначает число пороговых булевых функций от переменных $x_{1}, \ldots$, $x_{n}$. Имеется много статей, в которых исследуется поведение функции $T(n)$ (см., например, [334], [200], [84], [203], [204], [199], [335], [339], [23], [201], [113]) (ссылки даны в хронологическом порядке).

Верхняя оценка $\log _{2} T(n)<n^{2}$ была установлена несколькими авторами. Ее доказательство имеется, например, в [204]. Нижняя оценка $\log _{2} T(n)>n^{2}(1-10 / \log n)$ впервые была получена Ю. А. Зуевым [349] (см. также [350] и [351]).

Таким образом, при $n \rightarrow \infty$ справедливо асимптотическое равенство

$$
\log _{2} T(n) \sim n^{2}
$$

Число $T_{0}(n)$ пороговых монотонных булевых функций от $n$ переменных $x_{1}, \ldots, x_{n}$ связано с числом $T(n)$ неравенствами $T_{0}(n)<T(n)<2^{n} T_{0}(n)$.

Задачи о нахождении асимптотик для $T(n)$ и $T_{0}(n)$ оказались довольно сложными и до сих пор не решены.

Осветим более подробно связь между пороговьми монотонными булевыми функциями и системами линейных неравенств.

Из теоремы 1.1 и соотношения (3.4) следует, что среди функций из $M(n)$ и, следовательно, среди всех булевых функций от $n$ переменных пороговые функции составляют весьма малую долю. Однако любая булева функция $f$ от $n$ переменных может быть задана системой линейных неравенств

$$
\begin{aligned}
& a_{11} x_{1}+\cdots+a_{1 n} x_{n} \leqslant b_{1}, \\
& \ldots \ldots \ldots \ldots \ldots \ldots \ldots \ldots \cdots \\
& a_{m 1} x_{1}+\cdots+a_{m n} \leqslant b_{m}
\end{aligned}
$$

такой, что наборы из $E^{n}$, на которых $f$ равна 0 , и только они удовлетворяют этой системе. Такое задание булевой функции $f$ системой линейных неравенств (3.5) называется пороговым представлением функции $f$, а наименьшее число $m$ необходимых для этого неравенств - пороговым числом $t(f)$ функции $f$. 
Пороговое представление булевых функций возникло при решении задач булева линейного программирования, в частности задачи (3.1), (3.2).

Этим объясняется интерес к пороговьм представлениям монотонных булевых функций. В частности, в ряде работ исследовалась задача о величине порогового числа.

Так как каждая элементарная конъюнкция является пороговой функцией, а число верхних нулей любой функции из $M(n)$ не превосходит $\left(\begin{array}{c}n \\ n / 2\end{array}\right)$, то для каждой функции $f \in M(n)$ пороговое число $t(f) \leqslant\left(\begin{array}{c}n \\ n / 2\end{array}\right)$.

Используя информацию из [152] о структуре типичных функций из $§ 1.2$ и другие соображения, Ю. А. Зуев и Л. И. Липкин [353] доказали следуюшее утверждение.

Теорема 3.3. Для почти каждой функиии $f$ из $M(n)$ справедливо соотношенuе

$$
t(f)=\Theta\left(\left(\begin{array}{c}
n \\
n / 2
\end{array}\right) / n\right)
$$

Поведение параметра $t(f)$ и связь функций из $M(n)$ с системами линейных неравенств исследовалась также в работах [274], [345], [348], [352]-[356].

\section{§ 3.4. Тесты и монотонные булевы функции}

Имеются задачи, в которых по ряду известных признаков предмета или явления необходимо установить, обладает ли данньй объект некоторьми свойствами.

Например, при поиске неисправностей в дискретных схемах требуется выяснить, какие элементы работают правильно, а какие - неправильно. В химии важной является задача прогноза - обладает ли данное соединение свойством катализатора, в медицине по ряду симптомов важно установить, чем болеет пациент и т. п.

При решении таких задач полезным является понятие теста. По-видимому, строгое определение теста было введено в работе [38] в связи с использованием логических методов при поиске неисправностей в электрических схемах.

Группу из $r$ объектов, обладающих некоторыми признаками из $m$ заданных, можно представить в виде прямоугольной таблицы $\left(a_{i j}\right)$ размера $r \times m$. Строки этой таблицы соответствуют объектам, а столбцы - признакам. Элемент $a_{i j}$ означает, что $j$-й признак $i$-го объекта равен $a_{i j}$.

Одной из важнейших задач при таком подходе является описание заданной группы объектов минимальным числом признаков. Для многих задач такого вида достаточно рассматривать бинарные таблицы, заполненные элементами 0 и 1 (ниже будут рассматриваться только такие таблищы).

Пусть $T$ - бинарная прямоугольная таблица размера $r \times m$ с попарно различными строками. Набор столбцов $\left\langle i_{1}, \ldots, i_{k}\right\rangle$ таблицы $T$ назьвается тестом таблицы $T$, если подтаблица таблицы $T$, состоящая из этих столбцов, имеет попарно различные строки. Число столбцов в тесте назьвается длиной теста.

Тест таблицы $T$ назьвается тупиковым, если после удаления из него произвольного столбца получается таблица, не являющаяся тестом.

Среди тупиковых тестов находятся все минимальные тесты, т.е. тесты минимальной длины.

Ясно, что если подтаблица $T^{\prime}$ таблицы $T$ является тестом, то подтаблица, полученная из $T^{\prime}$ добавлением произвольного столбца из $T \backslash T^{\prime}$, также является тестом. Поэтому таблище $T$ можно поставить в соответствие следующую функцию $f_{T}\left(x_{1}, \ldots, x_{m}\right)$ 
из $M(m)$. Пусть $i_{1}, \ldots, i_{k}$ - номера столбцов, которые образуют тест в $T$, и $\widetilde{\sigma}=$ $\left(\sigma_{1}, \ldots, \sigma_{m}\right)$ - такой набор из $E^{m}$, что $\sigma_{i_{1}}=\cdots=\sigma_{i_{k}}=1$, а остальные компоненты набора $\widetilde{\sigma}$ равны 0 . Тогда $f_{T}\left(\sigma_{1}, \ldots, \sigma_{m}\right)=1$. Функция $f_{T}\left(x_{1}, \ldots, x_{m}\right)$ назьвается характеристической функцией тестов таблицы $T$. Эта функция содержит всю информацию о тестах таблицы $T$ : в частности, ее нижние единицы соответствуют тупиковым тестам таблицы $T$.

По этой причине иногда вместо тупиковых тестов таблицы изучают характеристическую функцию тестов этой таблицы.

Известно (см., например, [142]), что не всякая монотонная булева функция может быть характеристической для тестов какой-нибудь бинарной таблицы. Вместе с тем в [142] доказано следующее утверждение. Пусть $f\left(x_{1}, \ldots, x_{n}\right)$ - произвольная функция из $M(n)$ с $l$ верхними нулями и число $p$ таково, что $2^{p-1} \leqslant l<2^{p}$. Тогда существует такая бинарная прямоугольная таблица $T$ размера $(2 l+1) \times(n+p)$, что функция $f\left(x_{1}, \ldots, x_{n}\right) x_{n+1} \cdots x_{n+p}$ является характеристической функцией тестов таблицы $T$.

Приведем два примера задач, в которых используются тесты.

Поиск неисправностей в булевых схемах. Пусть булева схема $S$ имеет $n$ входов $x_{1}, \ldots, x_{n}$, один выход и вычисляет функцию $f\left(x_{1}, \ldots, x_{n}\right)$. Предположим, что в результате неправильной работы элементов схема $S$ может перейти в схемы $S_{1}^{\prime}, \ldots, S_{r}^{\prime}$, которые реализуют булевы функции $f_{1}^{\prime}\left(x_{1}, \ldots, x_{n}\right), \ldots, f_{r}^{\prime}\left(x_{1}, \ldots, x_{n}\right)$ соответственно. При этом допускается, что $f_{i}^{\prime} \equiv f_{j}^{\prime}$, т.е. две различные неисправности функционально неразличимы. Пусть $f_{1}, \ldots, f_{p}$ - совокупность булевых функций, характеризуюших попарно различные классы неисправностей, и пусть $f_{1}=f$. В этом случае получаем прямоугольную таблицу $T$, состояшую из $p$ строк и $2^{n}$ столбцов. Строки таблищы $T$ соответствуют классам функционально различимых неисправностей, а столбцы - наборам из $E^{n}$.

Для того чтобы ответить на вопрос, реализует ли схема функцию $f_{1}$ или какому классу принадлежит неисправность, достаточно на входы схемы подать наборы из $E^{n}$, образующие тест, и при каждом таком наборе определить значение выхода схемы.

Диагностика заболеваний. При диагностике определенных заболеваний появляется таблища, столбцы которой соответствуют видам заболеваний, а строки - симптомам (признакам). Если признаки проявляются дискретным образом (например, нормальна ли температура, нормально ли кровяное давление и т.п.), то получается бинарная таблища. Здесь интересны две задачи: а) установить, здоров ли данньй пациент, исходя из значений признаков заболеваний; b) установить конкретный диагноз.

Диагностические задачи медицины, как и многие другие, изучаются методами распознавания образов. На полезность использования тестов для решения таких задач, по-видимому, впервые было указано в работе [48]. Впоследствии тесты применялись во многих работах (см., например, [297], [347]).

Теперь сформулируем несколько конкретных результатов.

В. А. Варданян в работах [310]-[313] рассматривал следуюшую задачу. Пусть $f\left(x_{1}, \ldots, x_{n}\right)$ - произвольная булева функция. Множество наборов $T(f) \subseteq E^{n}$ называется единичным динамическим тестом функции $f\left(x_{1}, \ldots, x_{n}\right)$, если из сушественной зависимости функции $f$ от переменной $x_{i}, 1 \leqslant i \leqslant n$, следует 
сушествование набора $\widetilde{\alpha}=\left(\alpha_{1}, \ldots, \alpha_{n}\right) \in T(f)$ такого, что

$$
f\left(\alpha_{1}, \ldots, \alpha_{i-1}, \alpha_{i}, \alpha_{i+1}, \ldots, \alpha_{n}\right) \neq f\left(\alpha_{1}, \ldots, \alpha_{i-1}, \bar{\alpha}_{i}, \alpha_{i+1}, \ldots, \alpha_{n}\right)
$$

Для функщии $f$ требуется определить длину единичного динамического теста.

Используя информацию из [152] (см. $\S 1.2)$ о структуре типичных монотонных булевых функщий, В.А. Варданян [312] доказал, что для почти каждой функщии из $M(n)$ минимальньй единичный динамический тест состоит из двух наборов.

О. А. Долотова [49] изучала вычисление функций из $M(n)$ такими булевыми схемами, в которых все элементы работают без сбоев, а ошибки возникают только на входах схемы. При этом в каждой схеме ошибка может появиться только на одном произвольном входе: на такой вход вместо переменной поступает константа 0 или 1. Задача состоит в нахождении теста минимальной длины, которьй позволяет выяснить наличие неправильно работающего входа.

Используя информацию из [152] (см. 1 1.2) о структуре типичных монотонных булевых функций, О.А. Долотова показала, что для почти каждой функции из $M(n)$ длина минимального теста равна 4 при четном $n$ и равна 4 или 5 при нечетном $n$.

Подобные результаты имеются в работах [50] и [51] для остальных замкнутых классов монотонных булевых функций.

Н. П. Редькин [252] рассмотрел вычисление функций

$$
f_{2}\left(x_{1}, \ldots, x_{n}\right)=\bigvee_{1 \leqslant i<j \leqslant n} x_{i} x_{j}, \quad n \geqslant 2,
$$

булевьми схемами, которые состоят из надежных и ненадежных элементов, причем при неправильной работе не более $k$ произвольных ненадежных элементов схема вычисляет ту же функцию, что и при правильной работе всех элементов. Предполагается, что стоимость каждого надежного элемента равна $p>0$, а стоимость ненадежного элемента равна 1. Им доказаны следующие утверждения:

1) если $p>k+1$ и неправильно работающий ненадежный элемент может реализовать как константу 0 , так и константу 1 , то сложность минимальной (по стоимости) булевой схемы в базисе $\{\&, \vee,-\}$, вычисляющей функцию $f_{2}\left(x_{1}, \ldots, x_{n}\right)$, при $n \rightarrow \infty$ асимптотически равна $n(k+2)$;

2 ) если $p>0$ и неправильно работающий ненадежный элемент реализует константу 0 , то сложность минимальной схемы в базисе $\{\&, \vee\}$, вьгисляющей функцию $f_{2}\left(x_{1}, \ldots, x_{n}\right)$, при $n \rightarrow \infty$ асимптотически равна $n \cdot \min \{2 p, k+2\}$.

В работе [253] рассматривалась аналогичная задача вычисления последовательности других монотонных булевых функций посредством булевых схем без ветвлений при $k=1$.

\section{§3.5. Стековые фильтры}

Фильтр - устройство, осуществляющее определенное преобразование входных сигналов в выходные сигналы. Осушествляемое фильтром преобразование назьвается фильтрацией. Основной целью фильтрации является устранение шума из сигнала. 
Среди многих типов фильтров используются стековые фильтры. Они были предложены в работе [332]. Обзоры по стековым фильтрам имеются, например, в [224], [74] и $[344]$.

Стековые фильтры работают в скользяшем режиме, используя окно заданного размера. Известно несколько определений стековых фильтров с окном размера $n$. Мы воспользуемся следующим определением.

Пусть $k$ - произвольное натуральное число, $k<2^{n}$, и пусть $P_{1}, P_{2}, \ldots, P_{k}-$ заданные подмножества множества $\{1,2, \ldots, n\}$. Пусть $\mathbf{X}=\left(X_{1}, \ldots, X_{n}\right)$ - вещественнозначный вектор наблюдений, находящийся в окне фильтра. Тогда такой стековый фильтр осушествляет преобразование

$$
S(\mathbf{X})=\max \left\{\min \left\{X_{j} \mid j \in P_{1}\right\}, \ldots, \min \left\{X_{j} \mid j \in P_{k}\right\}\right\}
$$

Следовательно, множества $P_{1}, P_{2}, \ldots, P_{k}$ полностью определяют стековый фильтр. Ясно, что в общем случае операции max и $\min$ являются обобщениями операций дизъюнкции и конъюнкции в случае бинарной области. В этом случае стековый фильтр задается монотонной булевой функцией $f$ от $n$ переменных и поэтому в качестве $P_{1}, P_{2}, \ldots, P_{k}$ можно использовать только попарно несравнимые подмножества.

Статистические свойства стековых фильтров могут быть охарактеризованы посредством вероятностей выбора рангов, определяемых следующим образом. Пусть входной вектор $\mathbf{X}=\left(X_{1}, \ldots, X_{n}\right)$ состоит из независимых и одинаково распределенных случайных величин. Предполагается, что $X_{1}, \ldots, X_{n}$ упорядочены в порядке неубывания и $i$-й член в этом упорядочении обозначен через $X_{(i)}$. В этом случае $X_{(i)}$ называется $i$-й порядковой статистикой.

Так как выход стекового фильтра всегда совпадает с одним из своих входов, то величина

$$
p_{i}=\operatorname{Pr}\left\{S(\mathbf{X})=X_{(i)}\right\}, \quad 1 \leqslant i \leqslant n,
$$

называется вероятностью выбора $i$-го ранга. Вектор $\mathbf{p}=\left(p_{1}, \ldots, p_{n}\right)$ назьвается вектором вероятности выбора рангов. Из этого вектора можно извлечь важную информацию о чувствительности стекового фильтра к кратковременным всплескам на его входе.

Каждой функции $f \in M(n)$ ставятся в соответствие числа $A_{1}, A_{2}, \ldots, A_{n}$ такие, что

$$
A_{i}=\left|\left\{\widetilde{\alpha} \in E^{n, i} \mid f(\widetilde{\alpha})=0\right\}\right| .
$$

Известно, что

$$
p_{i}=\frac{A_{n-i}}{\left(\begin{array}{c}
n \\
n-i
\end{array}\right)}-\frac{A_{n-i+1}}{\left(\begin{array}{c}
n \\
n-i+1
\end{array}\right)}, \quad 1 \leqslant i \leqslant n .
$$

Из теорем 1.9-1.11 следует, что векторы выбора рангов для почти всех стековых фильтров с окном размера $n$ (почти всех функций из $M(n)$ ) при $n \rightarrow \infty$ имеют вид:

$$
\mathbf{p}=\left(0, \ldots, 0, p_{n / 2-1}, p_{n / 2}, p_{n / 2+1}, p_{n / 2+2}, 0, \ldots, 0\right)
$$

если $n$ четно, и либо

$$
\mathbf{p}=\left(0, \ldots, 0, p_{(n-1) / 2}, p_{(n+1) / 2}, p_{(n+3) / 2}, p_{(n+5) / 2}, 0, \ldots, 0\right)
$$


либо

$$
\mathbf{p}=\left(0, \ldots, 0, p_{(n-3) / 2}, p_{(n-1) / 2}, p_{(n+1) / 2}, p_{(n+3) / 2}, 0, \ldots, 0\right),
$$

если $n$ нечетно.

Отсюда видно, почему многие стековые фильтры являются нечувствительными к всплескам на своих входах: почти все стековые фильтры отбраковывают все порядковые статистики за исключением четырех средних.

Эти ненулевые вероятности выбора статистик обозначим через $p^{1}, p^{2}, p^{3}, p^{4}$. При четном $n$ пусть $F_{0}\left(n, p^{1}, p^{2}, p^{3}, p^{4}\right)$ обозначает вероятность того, что случайно выбранньй стековьй фильтр с окном размера $n$ имеет четыре ненулевые вероятности выбора рангов, равные $p^{1}, p^{2}, p^{3}, p^{4}$. При нечетном $n$ пусть $F_{1}\left(n, p^{1}, p^{2}, p^{3}, p^{4}\right)$ обозначает вероятность того, что случайно выбранньй стековый фильтр с окном размера $n$ имеет следуюшие ненулевые вероятности выбора рангов:

$$
p^{1}=p_{(n-1) / 2}, \quad p^{2}=p_{(n+1) / 2}, \quad p^{3}=p_{(n+3) / 2}, \quad p^{4}=p_{(n+5) / 2} .
$$

Наконец, при нечетном $n$ пусть $F_{2}\left(n, p^{1}, p^{2}, p^{3}, p^{4}\right)$ обозначает вероятность того, что случайно выбранньй стековый фильтр с окном размера $n$ имеет следуюшие ненулевые вероятности выбора рангов:

$$
p^{1}=p_{(n-3) / 2}, \quad p^{2}=p_{(n-1) / 2}, \quad p^{3}=p_{(n+1) / 2}, \quad p^{4}=p_{(n+3) / 2} .
$$

В работе [162; теоремы 4-6] (см. также [279]) доказаны следуюшие утверждения.

Tеорема 3.4. Пусть $n$ четно, и пусть

$$
\begin{aligned}
& p^{1}=\frac{1}{2^{n / 2+1}}+\frac{t+O(1)}{\left(\begin{array}{c}
n \\
n / 2-1
\end{array}\right)}, \\
& p^{2}=\frac{1}{2}-\frac{1}{2^{n / 2+1}}-\frac{u}{\left(\begin{array}{c}
n \\
n / 2
\end{array}\right)}-\frac{t+O(1)}{\left(\begin{array}{c}
n \\
n / 2+1
\end{array}\right)}, \\
& p^{3}=\frac{1}{2}-\frac{1}{2^{n / 2+1}}+\frac{u}{\left(\begin{array}{c}
n \\
n / 2
\end{array}\right)}-\frac{k+O(1)}{\left(\begin{array}{c}
n \\
n / 2-1
\end{array}\right)}, \\
& p^{4}=\frac{1}{2^{n / 2+1}}+\frac{k+O(1)}{\left(\begin{array}{c}
n \\
n / 2-1
\end{array}\right)}
\end{aligned}
$$

суть ненулевые вероятности выбора рангов, причем $|O(1)|<2$. Тогда при любых $k, t$, и таких, что $|k| \leqslant n 2^{n / 4},|t| \leqslant n 2^{n / 4},|u| \leqslant n 2^{n / 2}$, и $n \rightarrow \infty$ справедливо соотношение

$$
\begin{aligned}
F_{0}\left(n, p^{1}, p^{2}, p^{3}, p^{4}\right) \sim & \sqrt{2^{n+1} /\left(\pi^{3}\left(\begin{array}{c}
n \\
n / 2
\end{array}\right)^{3}\right)} \\
& \times \exp \left\{-\frac{2^{n / 2}}{\left(\begin{array}{c}
n \\
n / 2-1
\end{array}\right)}\left(k^{2}+t^{2}\right)-\frac{2 u^{2}}{\left(\begin{array}{c}
n \\
n / 2
\end{array}\right)}\right\} .
\end{aligned}
$$


ТеОРема 3.5. Пусть п нечетно, и пусть

$$
\begin{aligned}
p^{1} & =\frac{1}{2^{(n+1) / 2}}+\frac{t+O(1)}{\left(\begin{array}{c}
n \\
(n+1) / 2
\end{array}\right)}, \\
p^{2} & =\frac{1}{2}+\frac{n-5}{2^{(n+7) / 2}}-\frac{u}{\left(\begin{array}{c}
n \\
(n-1) / 2
\end{array}\right)}-\frac{t+O(n)}{\left(\begin{array}{c}
n \\
(n+1) / 2
\end{array}\right)}, \\
p^{3} & =\frac{1}{2}-\frac{n+7}{2^{(n+7) / 2}}+\frac{u}{\left(\begin{array}{c}
n \\
(n-1) / 2
\end{array}\right)}-\frac{k+O(n)}{\left(\begin{array}{c}
n \\
(n-3) / 2
\end{array}\right)}, \\
p^{4} & =\frac{1}{2^{(n+3) / 2}}+\frac{k+O(1)}{\left(\begin{array}{c}
n \\
(n-3) / 2
\end{array}\right)}
\end{aligned}
$$

суть ненулевые вероятности выбора рангов, причем $|O(1)|<2$ и $|O(n)|<2 n$. Тогда при любьх $k, t, u$ таких, что $|k| \leqslant n 2^{n / 4},|t| \leqslant n 2^{n / 4},|u| \leqslant n 2^{n / 2}$, u $n \rightarrow \infty$ справедливо соотношение

$$
\begin{aligned}
F_{1}\left(n, p^{1}, p^{2}, p^{3}, p^{4}\right) \sim & \frac{1}{2} \sqrt{2^{n+1} /\left(\pi^{3}\left(\begin{array}{c}
n \\
(n-1) / 2
\end{array}\right)^{3}\right)} \\
& \times \exp \left\{-\frac{2^{(n+1) / 2}}{\left(\begin{array}{c}
n \\
(n-3) / 2
\end{array}\right)} k^{2}-\frac{2^{(n-1) / 2}}{\left(\begin{array}{c}
n \\
(n+1) / 2
\end{array}\right)} t^{2}-\frac{2 u^{2}}{\left(\begin{array}{c}
n \\
(n-1) / 2
\end{array}\right)}\right\} .
\end{aligned}
$$

ТеОрема 3.6. Пусть $n$ нечетно, и пусть

$$
\begin{aligned}
p^{1} & =\frac{1}{2^{(n+3) / 2}}+\frac{t+O(1)}{\left(\begin{array}{c}
n \\
(n+3) / 2
\end{array}\right)}, \\
p^{2} & =\frac{1}{2}-\frac{n+7}{2^{(n+7) / 2}}-\frac{u}{\left(\begin{array}{c}
n \\
(n+1) / 2
\end{array}\right)}-\frac{t+O(n)}{\left(\begin{array}{c}
n \\
(n+3) / 2
\end{array}\right)}, \\
p^{3} & =\frac{1}{2}+\frac{n-5}{2^{(n+7) / 2}}+\frac{u}{\left(\begin{array}{c}
n \\
(n+1) / 2
\end{array}\right)}-\frac{k+O(n)}{\left(\begin{array}{c}
n \\
(n-1) / 2
\end{array}\right)}, \\
p^{4} & =\frac{1}{2^{(n+1) / 2}}+\frac{k+O(1)}{\left(\begin{array}{c}
n \\
(n-1) / 2
\end{array}\right)}
\end{aligned}
$$

суть ненулевье вероятности выббора рангов, причем $|O(1)|<2$ и $|O(n)|<2 n$. Тогда при любьх $k, t, u$ таких, что $|k| \leqslant n 2^{n / 4},|t| \leqslant n 2^{n / 4},|u| \leqslant n 2^{n / 2}$, u $n \rightarrow \infty$ справедливо соотношение

$$
\begin{aligned}
F_{2}\left(n, p^{1}, p^{2}, p^{3}, p^{4}\right) \sim & \frac{1}{2} \sqrt{2^{n+1} /\left(\pi^{3}\left(\begin{array}{c}
n \\
(n-1) / 2
\end{array}\right)^{3}\right)} \\
& \times \exp \left\{-\frac{2^{(n-1) / 2}}{\left(\begin{array}{c}
n \\
(n-1) / 2
\end{array}\right)} k^{2}-\frac{2^{(n+1) / 2}}{\left(\begin{array}{c}
n \\
(n+3) / 2
\end{array}\right)} t^{2}-\frac{2 u^{2}}{\left(\begin{array}{c}
n \\
(n-1) / 2
\end{array}\right)}\right\} .
\end{aligned}
$$

Результаты из [152] использовались в работе [278]. 


\section{СПИСОК ЛИТЕРАТУРЫ}

[1] M. Ajtai. $\Sigma_{1}^{1}$-formulae on finite structures // Ann. Pure Appl. Logic. 1983. V. 24. № 1. P. 1-48.

[2] M. Ajtai, Y. Gurevich. Monotone versus positive // J. ACM. 1987. V. 34. №4. P. 1004-1015.

[3] M. Ajtai, J. Komlós, E. Szemerédi. An $O(n \log n)$ sorting network // Proceedings of the 15th Annual ACM Symposium on Theory of Computing (Boston, 1983). New York: ACM Press, 1983. P. 1-9.

[4] В.Б. Алексеев. Расшифровка некоторых классов монотонных многозначных функций // Журн. вычисл. матем. и матем. физ. 1976. Т. 16. № 1. С. 189-198.

[5] N. Alon, R. B. Boppana. The monotone circuit complexity of Boolean functions // Combinatorica. 1987. V. 7. № 1. P. 1-22.

[6] K. Amano, A. Maruoka. Potential of the approximation method // Proceedings of the 37th Annual Symposium on Foundations of Computer Science (Burlington, October 14-16, 1996). Los Alamitos: IEEE Comput. Soc. Press, 1996. P. 431-440.

[7] K. Amano, A. Maruoka. Complexity of limited-negation circuits for monotone logic functions // Algorithms and Theory of Computing (Kyoto, 2-4 February 1998). Proceedings of symposium. Kyoto: Kyoto Univ., 1998. P. 71-78. (Sūrikaisekikenkyūsho Kōkyūroku. № 1041.) (на япон. яз.)

[8] K. Amano, A. Maruoka. A superpolynomial lower bound for a circuit computing the clique function with at most $(1 / 6) \log \log n$ negation gates // Mathematical Foundations of Computer Science 1998. Proceedings of the 23rd International Symposium MFCS'98 (Brno, August 24-28, 1998). Berlin: Springer-Verlag, 1998. P. 399-408. (Lecture Notes in Comput. Sci. V. 1450.)

[9] А. Е. Андреев. Об одном методе получения нижних оценок сложности индивидуальных монотонных функций // Докл. АН СССР. 1985. Т. 282. № 5. С. 1033-1037.

[10] А.Е. Андреев. О сложности монотонных функций // Вестник МГУ. Сер. 1. Матем., мех. 1985. № 4. С. 83-87.

[11] А. Е. Андреев. Об одном семействе булевых матриц // Вестник МГУ. Сер. 1. Матем., мех. 1986. № 2. С. $97-100$.

[12] А.Е. Андреев. Об одном методе получения эффективных нижних оценок монотонной сложности // Алгебра и логика. 1987. Т. 26. № 1. С. 3-26.

[13] А. Е. Андреев. Об одном методе получения более чем квадратичных эффективных нижних оценок сложности $\pi$-схем // Вестник МГУ. Сер. 1. Матем., мех. 1987. №1. С. 70-73.

[14] А. Е. Андреев. О синтезе схем из функциональных элементов в полных монотонных базисах // Математические вопросы кибернетики. Вып. 1. М.: Наука, 1988. С. 114-139.

[15] R. Balbes. On counting Sperner families // J. Combin. Theory. Ser. A. 1979. V. 27. № 1. P. 1-9.

[16] J. Berman, A. Burger, P. Köhler. The free distributive lattice on seven generators // Notices Amer. Math. Soc. 1975. V. 22. №6. P. A622.

[17] J. Berman, P. Köhler. Cardinalities of finite distributive lattices // Mitt. Math. Sem. Giessen. 1976. V. 121. P. 103-124.

[18] M. Beynon. Replacement in monotone Boolean networks: an algebraic perspective // Foundations of Software Technology and Theoretical Computer Science (Bangalore, 1984). Berlin: Springer-Verlag, 1984. P. 165-178. (Lecture Notes in Comput. Sci. V. 181.)

[19] M. Beynon. Replaceability and computational equivalence for monotone Boolean functions // Acta Inform. 1985. V. 22. № 4. P. 433-449.

[20] M. Beynon. Boolean function complexity: a lattice-theoretic perspective // Boolean Function Complexity (Durham, 1990). Cambridge: Cambridge Univ. Press, 1992. P. 35-56. (London Math. Soc. Lecture Note Ser. V. 169.)

[21] M. Beynon, J. Buckle. On the planar monotone computation of Boolean functions // Theoret. Comput. Sci. 1987. V. 53. № 2-3. P. 267-279. 
[22] J. C. Bioch, T. Ibaraki. Complexity of identification and dualization of positive Boolean functions // Inform. and Comput. 1995. V. 123. № 1. P. 50-63.

[23] M. Bloch, J. Morávek. Bounds of the number of threshold functions // Information Processing Machines, № 13. Prague, 1967. Р. 67-73. (Рус. пер.: М. Блох, Я. Моравек. Оценка числа пороговых функций // Кибернетический сборник. Нов. сер. Вып. 6. М.: Мир, 1969. C. $82-88$.)

[24] N. Blum. An $\Omega\left(n^{4 / 3}\right)$ lower bound on the monotone network complexity of the $n$-th degree convolution // Proceedings of the 22th Annual Symposium on Foundations of Computer Science (Nashville, 1981). New York: IEEE Comput. Soc. Press, 1981. P. 101-108.

[25] N. Blum. An $\Omega\left(n^{4 / 3}\right)$ lower bound on the monotone network complexity of the $n$th degree convolution // Theoret. Comput. Sci. 1985. V. 36. № 1. P. 59-69.

[26] R. B. Boppana. Threshold functions and bounded depth monotone curcuits // Proceedings of the Sixteenth Annual ACM Symposium on Theory of Computing (Washington, 1984). New York: ACM Press, 1984. P. 475-479.

[27] R. B. Boppana. Amplification of probabilistic Boolean formulas // Proceedings of the 26th Annual Symposium on Foundations of Computer Science (Portland, 1985). New York: IEEE Comput. Soc. Press, 1985. P. 20-29.

[28] R. B. Boppana. Threshold functions and bounded depth monotone curcuits // J. Comput. System Sci. 1986. V. 32. № 2. P. 222-229.

[29] R. B. Boppana. Amplification of probabilistic Boolean formulas // Advances in Computer Research. V. 5: Randomness and Computation. Greenwich: IAI Press, 1989. P. 27-45.

[30] R. B. Boppana, M. Sipser. The complexity of finite functions // Handbook of Theoretical Computer Science. V. A. Amsterdam: Elsevier, 1990. P. 757-804.

[31] E. Boros, P. L. Hammer, T. Ibaraki, K. Kawakami. Identifying 2-monotonic positive Boolean functions in polynomial time // ISA'91. Algorithms. Proceedings of the Second Annual International Symposium (Taipei, December 16-18, 1991). Berlin: Springer-Verlag, 1991. P. 104-115. (Lecture Notes in Comput. Sci. V. 557.)

[32] E. Boros, P. L. Hammer, T. Ibaraki, K. Kawakami. Polynomial-time recognition of 2-monotonic positive Boolean functions given by an oracle // SIAM J. Comput. 1997. V. 26. № 1. P. 93-109.

[33] S. Bublitz. Decomposition of graph and monotone formula size of homogeneous functions // Acta Inform. 1986. V. 23. № 6. P. 689-696.

[34] S. Bublitz, U.Schürfeld, B. Voigt, I. Wegener. Properties of complexity measures for PRAMs and WRAMs // Mathematical Foundations of Computer Science. 23rd International Symposium MFCS'96 (Bratislava, August 25-29, 1986). Berlin: Springer-Verlag, 1986. P. 230-238. (Lecture Notes in Comput. Sci. V. 233.)

[35] S. Bublitz, U. Schürfeld, I. Wegener. Properties of complexity measures for PRAMs and WRAMs // Theoret. Comput. Sci. 1986. V. 48. № 1. P. 53-73.

[36] L. Calabi, J. A. Riley. Inessentiality in minimal networks and formulas // IRE Trans. Electron. Comput. 1962. V. EC-11. № 5. P. 711-713.

[37] А. В. Чашкин. О влиянии отрицаний на сложность реализации частичных монотонных булевых функций // Вестник МГУ. Сер. 1. Матем., мех. 1995. № 3. С. 44-47.

[38] И. А. Чегис, С. В. Яблонский. Логические способы контроля работы электрических схем // Труды МИАН. 1958. Т. 51. С. 270-360.

[39] Д. Ю. Черухин. Нижние оценки формальной сложности симметрических булевых функций // Дискрет. анализ и исслед. операций. Сер. 1. 2000. Т. 7. № 3. С. 86-98.

[40] R. Church. Numerical analysis of certain free distributive structures // Duke Math. J. 1940. V. 6. № 3. P. 732-734.

[41] R. Church. Enumeration by rank of the elements of the free distributive lattices with seven generators // Notices Amer. Math. Soc. 1965. V. 12. №6. P. 724.

[42] B. Commentz-Walter. Size-depth trade-off in Boolean formulas // Automata, Languages and Programming. Proceedings of the Fifth Colloquium (Udine, July 17-21, 1978). Berlin: Springer-Verlag, 1978. P. 125-141. (Lecture Notes in Comput. Sci. V. 62.) 
[43] B. Commentz-Walter. Size-depth trade-off in monotone Boolean formulae // Acta Inform. 1979. V. 12. № 3. P. 227-243.

[44] B. Commentz-Walter, J. Sattler. Size-depth tradeoff in nonmonotone Boolean formulae // Acta Inform. 1980. V. 14. № 3. P. 257-269.

[45] D. Coppersmith, B. Shieber. Lower bounds on the depth of monotone arithmetic computations // Proceedings of the 33rd Annual Symposium on Foundations of Computer Science (Pittsburgh, 1992). Los Alamitos: IEEE Comput. Soc. Press, 1992. P. 288-295.

[46] D. W. Dai. A kind of matrix representation of a free distributive lattice and evaluation of its order // Wuhan Daxue Xuebao. 1980. № 3. P. 39-50.

[47] R. Dedekind. Über Zerlegungen von Zahlen durch ihre grössten gemeinsamen Teiler // Festschrift der Techn. Hochsch. Braunschweig bei Gelegenheit der 69. Versammlung deutscher und Ärzte, 1897. P. 1-40.

[48] А. Н. Дмитриев, Ю.И. ЖКуравлев, Ф. П. Кренделев. О математических принципах классификации предметов и явлений // Дискретный анализ: Сб. науч. тр. Вып. 7. Новосибирск: Ин-т математики СО АН СССР, 1966. С. 3-15.

[49] О.А. Долотова. О сложности проверяющих тестов // Конструкции в алгебре и логике. Тверь: Тверской ун-т, 1990. С. 43-49.

[50] О.А. Долотова. О сложности минимальных проверяющих тестов для классов Поста // Докл. АН СССР. 1992. Т. 324. № 4. С. 730-733.

[51] О.А. Долотова. О минимальных проверяющих тестах функций из классов Поста // Дискрет. матем. 1993. Т. 5. № 2. С. 75-82.

[52] M. Dubiner, U. Zwick. Amplification and percolation // Proceedings of the 33rd Annual Symposium on Foundations of Computer Science (Pittsburgh, 1992). Los Alamitos: IEEE Comput. Soc. Press, 1992. P. 258-267.

[53] P.E. Dunne. Lower bounds on the monotone network complexity of threshold functions // Proceedings of the 22nd Annual Allerton Conference on Communication, Control and Computation. Urbana-Champaign: Univ. of Illinois, 1984. P. 911-920.

[54] P.E. Dunne. A 2.5n lower bound on the monotone network complexity of $T_{3}^{n} / /$ Acta Inform. 1985. V. 22. № 2. P. 229-240.

[55] P. E. Dunne. The Complexity of Boolean Networks. London: Academic Press, 1988.

[56] P.E. Dunne. On monotone simulations of nonmonotone networks // Theoret. Comput. Sci. 1989. V. 66. № 1. P. 15-25.

[57] P.E. Dunne. Superpolynomial lower bounds on monotone network complexity // Theoretical foundations of VLSI desing. Cambridge: Cambridge Univ. Press, 1990. P. 403-438. (Cambridge Tracts Theoret. Comput. Sci. V. 10.)

[58] P.E. Dunne. Relationships between monotone and non-monotone network complexity // Boolean Function Complexity (Durhan, 1990). Cambridge: Cambridge Univ. Press, 1992. P. 1-24. (London Math. Soc. Lecture Note Ser. V. 169.)

[59] P.E. Dunne. Ceilings of monotone Boolean functions // J. Universal Comput. Sci. 1996. V. 2. № 7. P. 533-548.

[60] J. Edmonds, R. Impagliazzo, S. Rudich, J. Sgall. Communication complexity towards lower bounds on circuit depth // Proceedings of the 32nd Annual Symposium on Foundations of Computer Science (San Juan, October 1-4, 1991). Los Alamitos: IEEE Comput. Soc. Press, 1991. P. 249-257.

[61] K. Engel. Sperner Theory. Cambridge: Cambridge Univ. Press, 1997. (Encyclopedia Math. Appl. V. 65.)

[62] K. Engel, H.-D.O. F. Grönau. Sperner Theory in Partially Ordered Sets. Leipzig: Teubner, 1985. (Teubner-Texte Math. V. 78.)

[63] P. Erdös, D. J. Kleitman. Extremal problems among subsets of a set // Discrete Math. 1974. V. 8. № 3. Р. 281-294. (Рус. пер.: П. Эрдёш, Дж. Спенсер. Вероятностные методы в комбинаторике. М.: Мир, 1976. С. 115-130.)

[64] В. Феллер. Введение в теорию вероятностей и ее приложения. Т. 1. М.: Мир, 1984.

[65] R. Fidytek, A. W. Mostowski, R. Somla, A. Szepietowski. Algorithms counting monotone Boolean functions // Inform. Process. Lett. 2001. V. 79. № 5. P. 203-209. 
[66] M. Fischer. The complexity of negation-limited networks - a brief survey // Automata Theory and Formal Languages. Berlin: Springer-Verlag, 1975. P. 71-82. (Lecture Notes in Comput. Sci. V. 33.)

[67] M.J. Fischer, A.R. Meyer. Boolean matrix multiplication and transitive closure // Conference record 1971 12th Annual Symposium on Switching and Automata Theory (East Lansing, 1971). New York: IEEE, 1971. P. 129-131.

[68] M. J. Fischer, A.R. Meyer, M.S. Paterson. $\Omega(n \log n)$ lower bounds on length of Boolean formulas // SIAM J. Comput. 1982. V. 11. № 3. P. 416-427.

[69] M. L. Fred man, L. Khachiyan. On the complexity of dualization of monotone disjunctive normal forms // J. Algorithms. 1996. V. 21. № 3. P. 618-628.

[70] J. Friedman. Constructing $O(n \log n)$ size monotone formulae for the $k$-th elementary symmetric polynomial of $n$ Boolean variables // Proceedings of the 25th Annual Symposium on Foundations of Computer Science (Singer Island, 1984). New York: IEEE Comput. Soc. Press, 1984. P. 506-515.

[71] J. Friedman. Constructing $O(n \log n)$ size monotone formulae for the $k$-th threshold function of $n$ Boolean variables // SIAM J. Comput. 1986. V. 15. № 3. P. 641-654.

[72] M. Furst, J. Saxe, M. Sipser. Parity, circuits, and the polynomial time hierarchy // Proceedings of the 22nd Annual Symposium on Foundations of Computer Science (Nashville, 1981). New York: IEEE Comput. Soc. Press, 1981. P. 260-270.

[73] M. Furst, J. Saxe, M. Sipser. Parity, circuits, and the polynomial-time hierarchy // Math. Systems Theory. 1984. V. 17. № 1. P. 13-27.

[74] M. Gabbouj, E. Coyle, N. Gallager, Jr. An overview of median and stack filtering // Circuits Systems Signal Process. 1992. V. 11. № 1. P. 7-45.

[75] Д.Н. Гайнанов. Об одном критерии оптимальности алгоритма расшифровки монотонных булевых функций // Журн. вычисл. матем. и матем. физ. 1984. Т. 24. № 8. С. 1250-1257.

[76] G. Galbiati, M. Fischer. On the complexity of 2-output Boolean networks // Theoret. Comput. Sci. 1981. V. 16. №2. P. 177-185.

[77] D. R. Gaur, R. Krishnamurti. Self-duality of bouded monotone Boolean functions and related problems // Algorithmic learning theory. Proceedings of the 11th International Conference ALT 2000 (Sydney, December 11-13, 2000). Berlin: Springer-Verlag, 2000. P. 209-223. (Lecture Notes in Comput. Sci. V. 1968.)

[78] E. N. Gilbert. Lattice theoretic properties of frontal switching functions // J. Math. Phys. 1954. V. 33. №1. Р. 57-67. (Рус. пер.: Э.Н.Гилберт. Теоретико-структурные свойства замыкающих переключательных функций // Кибернетический сборник. Вып. 1. М.: ИЛ, 1960. C. $175-188$.

[79] M. Goldmann, J. Håstad. A simple lower bound for monotone clique using a communication game // Inform. Process. Lett. 1992. V. 41. № 4. P. 221-226.

[80] M. Goldmann, J. Håstad. Monotone circuits for connectivity have depth $(\log n)^{2-o(1)}$ (extended abstract) // Proceedings of the 27th Annual ACM Symposium on Theory of Computing (Las Vegas, 1995). New York: ACM Press, 1995. P. 569-574.

[81] M. Goldmann, J. Håstad. Monotone circuits for connectivity have depth $(\log n)^{2-o(1)}$ // SIAM J. Comput. 1998. V. 27. № 5. P. 1283-1294.

[82] L. M. Goldschlager. A space efficient algorithm for the monotone planar circuit value problem // Inform. Process. Lett. 1980. V. 10. № 1. P. 25-27.

[83] Ю. М. Горчаков. О числе монотонных функций // Конструкции в алгебре и логике. Тверь: Тверской ун-т, 1990. С. 22-24.

[84] E. Goto, H. Takahasi. Some theorems useful in threshold logic for enumerating Boolean functions // Inform. Processing 1962. Amsterdam: North-Holland, 1963. P. 747-752.

[85] M. Grigni, M.Sipser. Monotone complexity // Boolean Function Complexity (Durhan, 1990). Cambridge: Cambridge Univ. Press, 1992. P. 57-75. (London Math. Soc. Lecture Note Ser. V. 169.)

[86] Д. Ю. Григорьев. Об одной нижней оценке сложности вычисления семейства дизъюнкций в монотонном базисе // Зап. науч. семинаров ЛОМИ. 1977. Т. 68. С. 19-25. 
[87] М.И. Гринчук. О монотонной сложности пороговых функций // Методы дискретного анализа в теории графов: Сб. науч. тр. Вып. 52. Новосибирск: Ин-т математики СО РАН, 1992. C. $41-48$.

[88] V. Grolmusz. A weight-size trade-off for circuits with MODm gates // Proceedings of the 26th Annual ACM Symposium on Theory of Computing (Montréal, 1994). New York: ACM Press, 1994. P. 68-74.

[89] V. Gurvich, L. Khachiyan. On generating the irredundant conjunctive and disjunctive normal forms of monotone Boolean functions // Discrete Appl. Math. 1999. V. 96/97. P. $363-373$.

[90] A. Haken. Counting bottlenecks to show monotone $\mathrm{P} \neq \mathrm{NP} / /$ Proceedings of the 36th Annual Symposium on Foundations of Computer Science (Milwankee, October 23-25, 1995). Los Alamitos: IEEE Comput. Soc. Press, 1995. P. 36-40.

[91] A. Haken, S. A. Cook. An exponential lower bound for the size of monotone real circuits // J. Comput. System Sci. 1999. V. 58. № 2. P. 326-335.

[92] M. M. Halldórsson, J. Radkhakrishnan, K. V. Subrahmanyam. Directed vs. undirected monotone contact networks for threshold functions // Proceedings of the 34th Annual Symposium on Foundations of Computer Science (Palo Alto, 1993). Los Alamitos: IEEE Comput. Soc. Press, 1993. P. 604-613.

[93] G. Hansel. Nombre minimal de contacts de fermeture nécessaires pour réaliser une fonction booléenne symétrique de $n$ variables // C. R. Acad. Sci. Paris. 1964. V. 258. № 25. P. 6037-6040. (Рус. пер.: Ж. Ансель. Минимальное число замькающих контактов, достаточное для реализации одной симметричной булевой функции $n$ переменных // Кибернетический сборник. Нов. сер. Вып. 5. М.: Мир, 1968. С. 47-52.)

[94] G. Hansel. Nombre de lettres nécessaire pour écrire une fonction symétrique de $n$ variables // C. R. Acad. Sci. Paris. 1965. V. 261. № 21. P. 4297-4300.

[95] G. Hansel. Résultats concernant le nombre minimal de contacts nécessaire pour réaliser certaines fonctions booléennes symétriques // C. R. Acad. Sci. Paris. Ser. A-B. 1966. V. 262. № 12. P. A679-A681.

[96] G. Hansel. Sur le nombre des fonctions booléennes monotones de $n$ variables // C. R. Acad. Sci. Paris. Sér. A-B. 1966. V. 262. № 20. P. A1088-A1090. (Рус. пер.: Ж. Ансель. О числе монотонных булевых функций $n$ переменных // Кибернетический сборник. Нов. сер. Вып. 5. М.: Мир, 1968. С. 53-57.)

[97] G. Hansel. Construction d'un schéma de contacts bipolaire pour une fonction booléenne isotone arbitraire de $n$ variables // C. R. Acad. Sci. Paris. Sér. A-B. 1966. V. 263. № 19. P. A651-А654. (Рус. пер.: Ж. Ансель. Построение двухполюсной схемы для произвольной монотонной булевой функции $n$ переменных // Кибернетический сборник. Нов. сер. Вып. 15. М.: Мир, 1968. С. 58-63.)

[98] D. Harnik, R. Raz. Higher lower bounds on monotone size // Proceedings of the 32nd Annual ACM Symposium on Theory of Computing (Oregon, 2000). New York: ACM Press, 2000. P. 378-387.

[99] L. H. Harper, J. E. Sawage. On the complexity of the marriage problem // Adv. Math. 1972. V. 9. № 3. P. 299-312.

[100] J. Håstad. Almost optimal lower bounds for small depth circuits // Proceedings of the 18th Annual ACM Symposium on Theory of Computing (Berkeley, 1986). New York: ACM Press, 1986. P. 6-20.

[101] J. Håstad. Almost optimal lower bounds for small depth curcuits // Advances in Computer Research. V. 5: Randomness and Computation. Greenwich: IAI Press, 1989. P. 143-170.

[102] J. Håstad. The shrinkage exponent is $2 / /$ Proceedings of the 34th Annual Symposium on Foundations of Computer Science (Palo Alto, 1993). Los Alamitos: IEEE Comput. Soc. Press, 1993. P. 114-123.

[103] J. Håstad. The shrinkage exponent of De Morgan formulas is 2 // SIAM J. Comput. 1998. V. 28. № 1. P. 48-64. 
[104] J. Håstad, M. Goldmann. On the power of small-depth threshold circuits // Proceedings of the 31st Annual Symposium on Foundations of Computer Science (St. Louis, 1990). V. 2. Los Alamitos: IEEE Comput. Soc. Press, 1990. P. 610-618.

[105] J. Håstad, M. Goldmann. On the power of small-depth threshold circuits // Comput. Complexity. 1991. V. 1. № 2. P. 113-129.

[106] J. E. Hopcroft, R. M. Karp. An $n^{5 / 2}$ algorithm for maximum matchings in bipartite graphs // SIAM J. Comput. 1973. V. 2. № 4. P. 225-231.

[107] J. Hromkovič. On the number of monotonic Boolean functions // Comput. Artificial Intelligence. 1984. V. 3. P. 319-329.

[108] J. Hromkovič. On the number of monotonic functions from two-valued logic to $k$-valued logic // Kybernetika (Prague). 1985. V. 21. № 3. P. 228-234.

[109] S. B. Hu, D. W. Dai, C. C. Liu. An algorithm for solving Dedekind's problem by reduction of rank // Wuhan Daxue Xuebao. 1982. № 2. P. 31-36.

[110] R. Impagliazzo, N. Nisan. The effect of random restrictions on formula size // Random Structures Algorithms. 1993. V. 4. № 2. P. 121-133.

[111] R. Impagliazzo, R. Paturi, M.E. Saks. Size-depth trade-off for threshold circuits // Proceedings of the 25th Annual ACM Symposium on Theory of Computing (San Diego, 1993). New York: ACM Press, 1993. P. 541-550.

[112] R. Impagliazzo, R. Paturi, M. E. Saks. Size-depth tradeoffs for threshold circuits // SIAM J. Comput. 1997. V. 26. № 3. P. 693-707.

[113] А. А. Ирматов. Оценки числа пороговых функций // Дискрет. матем. 1996. Т. 8. № 4. C. $92-107$.

[114] M. Karchmer. On proving lower bounds for circuit size // Proceedings of the Eighth Annual Structure in Complexity Theory Conference (San Diego, May 18-21, 1993). Los Alamitos: IEEE Comput. Soc. Press, 1993. P. 112-118.

[115] M. Karchmer, R. Raz, A. Wigderson. On proving super-logarithmic depth lower bounds via the direct sum in communication complexity // Proceedings of the 6th Annual Structure in Complexity Theory Conference (Chicago, 1991). Los Alamitos: IEEE Comput. Soc. Press, 1991. P. 299-304.

[116] M. Karchmer, A. Wigderson. Monotone curcuits for connectivity require super-logarithmic depth // Proceedings of the 20th Annual ACM Symposium on Theory of Computing (Chicago, 1988). New York: ACM Press, 1988. P. 539-550.

[117] M. Karchmer, A. Wigderson. Monotone curcuits for connectivity require super-logarithmic depth // SIAM J. Discrete Math. 1990. V. 3. № 2. P. 255-265.

[118] Н. А. Карпова. О контактных схемах для монотонных функций // Докл. АН СССР. 1958. T. 123. № 1. C. 25-27.

[119] Н. А. Карпова. Минимальные схемы из замькающих контактов для монотонных булевых функций пяти переменных // Проблемы кибернетики. Вьп. 26. М.: Наука, 1973. C. 53-94.

[120] О. М. Касим-Заде. О неявной выразимости булевых функций // Вестник МГУ. Сер. 1. Матем., мех. 1995. № 2. С. 44-49.

[121] О.М.Касим-Заде. О неявной выразимости в двухзначной логике и криптоизоморфизмах двухэлементных алгебр // Докл. РАН. 1996. Т. 348. №3. С. 299-301.

[122] О. М. Касим-Заде. Об одной метрической характеристике неявных и параметрических представлений булевых функций // Математические вопросы кибернетики. Вып. 6. М.: Наука, 1996. С. 133-188.

[123] Н.Н.Катериночкина. Поиск максималњного верхнего нуля монотонной функции алгебры логики // Докл. АН СССР. 1975. Т. 224. №3. С. 557-560.

[124] Н. Н. Катериночкина. Поиск максимального верхнего нуля для одного класса монотонных функций $k$-значной логики // Докл. АН СССР. 1977. Т. 234. № 4. С. 746-749.

[125] Н. Н. Катериночкина. Поиск максимального верхнего нуля для некоторых подклассов монотонных булевых функций // Журн. вычисл. матем. и матем. физ. 1978. Т. 18. № 2. C. $516-519$. 
[126] Н.Н.Катериночкина. Поиск максималњного верхнего нуля дискретной монотонной функции // Rostock. Math. Kolloq. 1982. № 21. P. 5-10.

[127] Н. Н. Катериночкина. О задаче поиска максималшного верхнего нуля для ряда подклассов монотонных булевых функций // Журн. вычисл. матем. и матем. физ. 1987. Т. 27. №9. C. $1402-1411$.

[128] Н.Н. Катериночкина. Поиск максимального верхнего нуля для некоторых классов монотонных булевых функций из классификации Поста // Журн. вычисл. матем. и матем. физ. 1988. Т. 28. № 9. С. 1397-1406.

[129] Л.С. Хасин. Оценки сложности реализации монотонных симметрических функций в базисах $\vee, \&,-$ // Докл. АН СССР. 1969. Т. 189. № 4. С. 752-755.

[130] Л. С. Хасин. О реализации монотонных симметрических функций формулами в базисах $\vee, \&,-/ /$ Проблемы кибернетики. Вып. 21. М.: Наука, 1969. С. 253-257.

[131] Л. С. Хасин. Об использовании отрицания для реализации монотонных симметрических функций алгебры логики формулами в базисах $\vee, \&,-/ /$ Дискретный анализ: Сб. науч. тр. Вып. 17. Новосибирск: Ин-т математики СО АН СССР, 1970. С. 45-55.

[132] В. М. Храпченко. О сложности реализации симметрических функций формулами // Матем. заметки. 1972. Т. 11. №1. С. 109-120.

[133] В. М. Храпченко. Нижние оценки сложности схем из функционалшных элементов (обзор) // Кибернетический сборник. Нов. сер. Вып. 21. М.: Мир, 1984. С. 3-54.

[134] A. Kisielewicz. A solution of Dedekind's problem on the number of isotone Boolean functions // J. Reine Angew. Math. 1988. V. 3. №1. P. 1-6.

[135] M. Klawe, W. J. Paul, N. Pippenger, M. Yannakakis. On monotone formulae with restricted depth // Proceedings of the 16th Annual ACM Symposium on Theory of Computing (Washington, 1984). New York: ACM Press, 1984. P. 480-487.

[136] M. Kleiman, N. Pippenger. An explicit constructing of short monotone formulae for the monotone symmetric functions // Theoret. Comput. Sci. 1978. V. 7. № 3. P. 325-332.

[137] D. Kleitman. On Dedekind's problem: the number of monotone Boolean functions // Proc. Amer. Math. Soc. 1969. V. 21. № 3. Р. 677-682. (Рус. пер.: Д. Клейтман. О проблеме Дедекинда: число монотонных булевых функций // Кибернетический сборник. Нов. сер. Вып. 7. М.: Мир, 1970. С. 43-52.)

[138] D. Kleitman. The number of Sperner families of subsets of an $n$ element set // Infinite and finite sets (Colloq., Keszhely, 1973). V. 2. Amsterdam: North-Holland, 1975. P. 989-1001. (Colloq. Math. Soc. János Bolyai. V. 10.)

[139] D. Kleitman, G. Markowsky. On Dedekind's problem: the number of monotone Boolean functions. II // Trans. Amer. Math. Soc. 1975. V. 213. P. 373-390.

[140] Б. С. Кочкарев. Оценки сложности формул для монотонных функций алгебры логики в классе дизъюнктивных нормальных форм (д.н.ф.) // Учен. зап. Казан. ун-та. 1965. Т. 125. №6. C. $49-57$.

[141] M. Kochol. Efficient monotone circuits for threshold functions // Inform. Process. Lett. 1989. V. 32. № 3. P. 121-122.

[142] В. И. Колпаков. О соответствии между монотонными функциями и множеством тупиковых тестов для таблиц // Дискретньй анализ: Сб. науч. тр. Вып. 16. Новосибирск: Ин-т математики СО АН СССР, 1970. С. 44-50.

[143] В. К. Коробков. Реализация симметрических функций в классе $\pi$-схем // Докл. АН CCCP. 1956. T. 109. № 2. C. 260-263.

[144] В.К. Коробков. Оценка числа монотонных функций алгебры логики и сложности разрешающего алгоритма отыскания разрешающего множества для произволнной монотонной функции алгебры логики // Докл. АН СССР. 1963. Т. 150. № 4. С. 744-747.

[145] В.К. Коробков. К вопросу о числе монотонных функций алгебры логики // Дискретньй анализ: Сб. науч. тр. Вьп. 1. Новосибирск: Ин-т математики СО АН СССР, 1963. C. $24-27$.

[146] В. К. Коробков. О монотонных функциях алгебры логики // Проблемы кибернетики. Вып. 13. М.: Наука, 1965. С. 5-28. 
[147] В.К.Коробков. Некоторые обобщения задачи "расшифровки" монотонных функций алгебры логики // Дискретный анализ: Сб. науч. тр. Вып. 5. Новосибирск: Ин-т математики СО АН СССР, 1965. С. 19-25.

[148] В. К. Коробков. О некоторых целочисленных задачах линейного программирования // Проблемы кибернетики. Вып. 14. М.: Наука, 1965. С. 297-299.

[149] В.К. Коробков, Т. Л. Резник. О некоторых алгоритмах вычисления монотонных функций алгебры логики // Докл. АН СССР. 1962. Т. 147. № 5. С. 1022-1025.

[150] В. В. Коротких. О связи задач оптимального восстановления одного функционального класса с расшифровкой монотонных функций конечнозначной логики // Методы исследования сложных систем: Труды конференции молодых ученых ВНИСИ. М.: ВНИСИ, 1983. C. $18-24$.

[151] А. Д. Коршунов. Решение проблемы Дедекинда о числе монотонных булевых функций // Докл. АН СССР. 1977. Т. 233. № 4. С. 543-546.

[152] А. Д. Коршунов. О числе монотонных булевых функций // Проблемы кибернетики. Вып. 38. М.: Наука, 1981. С. 5-108.

[153] А. Д. Коршунов. О сложности кратчайших дизъюнктивных нормалшных форм случайных булевых функций // Методы дискретного анализа в оптимизации управляющих систем: Сб. науч. тр. Вып. 40. Новосибирск: Ин-т математики СО АН СССР, 1983. С. 25-53.

[154] А. Д. Коршунов. О числе $r$-элементных подмножеств в $E^{n}$ с равномощными границами. I // Методы дискретного анализа в теории графов и схем: Сб. науч. тр. Вып. 42. Новосибирск: Ин-т математики СО АН СССР, 1985. С. 44-61.

[155] А. Д. Коршунов. О числе $r$-элементных подмножеств в $E^{n}$ с равномощными границами. II // Методы дискретного анализа в теории графов и схем: Сб. науч. тр. Вып. 44. Новосибирск: Ин-т математики СО АН СССР, 1986. С. 24-53.

[156] А. Д. Коршунов. О мощности и структуре некоторых замкнутых классов Поста (семейств подмножеств конечного множества) // Докл. АН СССР. 1987. Т. 295.№ 3. C. $533-537$.

[157] A. D. Korshunov. The number and the structure of typical Sperner and $k$-non-separable families of subsets of a finite set // Foundamentals of Computation Theory (Kazan, June 22-26, 1987). Berlin: Springer-Verlag, 1987. P. 239-243. (Lecture Notes in Comput. Sci. V. 278.)

[158] А. Д. Коршунов. О мощности и структуре некоторых замкнутых классов Поста (семейств подмножеств конечного множества) // Модели и методы оптимизации. Новосибирск: Наука, 1988. С. 159-204. (Тр. АН СССР. Сиб. отд-ние. Ин-т математики. Т. 10.)

[159] A. D. Korshunov. Families of subsets of a finite set and closed classes of Boolean functions // Extremal Problems for Finite Sets (Visegrád, 1991). Budapest: János Bolyai Math. Soc., 1994. P. 375-396. (Bolyai Soc. Math. Stud. V. 3.)

[160] А. Д. Коршунов. О числе и строении монотонных булевых функций // Дискретная математика и ее приложения: Сборник лекций молодежных научных школ по дискретной математике и ее приложениям. М.: Изд-во Центра прикладных исследований при мех.-матем. факултете МГУ, 2001. С. 34-58.

[161] А. Д. Коршунов, А. А. Сапоженко. О числе двоичных кодов с расстоянием 2 // Проблемы кибернетики. Вып. 40. М.: Наука, 1983. С. 111-130.

[162] А. Д. Коршунов, И. Шмулевич. Число специальных монотонных булевых функций и статистические свойства стековых фильтров // Дискрет. анализ и исслед. операций. Сер. 1. 2000. T. 7. №3. C. 17-44.

[163] A. D. Korshunov, I. Shmulevich. On the distribution of the number of monotone Boolean functions relative to the number of lower units // Discrete Math. 2002. V. 257. № 2-3. P. 463-479.

[164] Д. Л. Кожевников, О.И.Ларичев. Сравнение алгоритмов расшифровки монотонных функций методом статистического моделирования // Журн. вычисл. матем. и матем. физ. 1999. Т. 39. № 8. С. 1415-1421. 
[165] Е. Г. Красулина. О реализации монотонных симметрических функций алгебры логики контактными схемами // Вестник МГУ. Сер. 13. Вычисл. матем. и кибернетика. 1987. № 2. C. $69-71$.

[166] Е. Г. Красулина. О сложности реализации монотонных симметрических функций алгебры логики контактными схемами // Математические вопросы кибернетики. Вып. 1. М.: Наука, 1988. С. 140-167.

[167] Р. Е. Кричевский. Сложность контактных схем, реализующих одну функцию алгебры логики // Докл. АН СССР. 1963. Т. 151. № 4. С. 803-806.

[168] Р. Е. Кричевский. Оценка сложности $\pi$-схемы для одной функции алгебры логики // Дискретньй анализ: Сб. науч. тр. Вып. 1. Новосибирск: Ин-т математики СО АН СССР, 1963. C. $13-23$.

[169] Р. Е. Кричевский. О сложности параллельно-последовательных контактных схем, реализующих одну последовательность булевых функций // Проблемы кибернетики. Вып. 12. M.: Наука, 1964. С. 45-55.

[170] Р. Е. Кричевский. Минимальная схема из замькающих контактов для одной функции алгебры логики // Дискретный анализ: Сб. науч. тр. Вып. 5. Новосибирск: Ин-т математики СО АН СССР, 1965. С. 89-92.

[171] А. В. Кузнецов. О средствах для обнаружения невыводимости или невыразимости // Логический вывод. М.: Наука, 1979. С. 5-53.

[172] E. A. Lamagna. The complexity of monotone networks for certain bilinear forms, routing problems, sorting, and merging // IEEE Trans. Comput. 1979. V. C-28. № 10. P. 773-782.

[173] E. A. Lamagna, J. E. Savage. Combinatorial complexity of some monotone functions // Proceedings of the 15th Annual Symposium on Switching and Automata Theory (New Orleans, 1974). Long Beach: IEEE Comput. Soc. Press, 1974. P. 140-144.

[174] C. C. Liu. The mechanical algorithm for solving Dedekind's problem // Wuhan Daxue Xuebao. 1980. № 3. P. 30-38.

[175] L. Lovász. The works of A. A. Razborov // Proceedings of the International Congress of Mathematicians (Kyoto, August 21-29, 1990). V. 1. Tokyo: Math. Soc. Japan, 1991. Р. 37-40. (Рус. пер.: Л. Ловас. О работах А.А. Разборова // Международный конгресс математиков в Киото, 1990. Избранные доклады. М.: Мир, 1996. С. 52-58.)

[176] С. А. Ложкин. О связи между глубиной и сложностью эквивалентных формул и о глубине монотонных функций алгебры логики // Проблемы кибернетики. Вып. 38. М.: Наука, 1981. C. $269-271$.

[177] С. А. Ложкин, А. А. Семенов. Об одном методе сжатия информации и о сложности реализации монотонных симметрических функций // Изв. вузов. Матем. 1988. № 7 . C. $44-52$.

[178] D. Lubell. A short proof of Sperner's lemma // J. Combin. Theory. 1966. V. 1. № 2. P. 299.

[179] F. Lunnon. The IU function: The size of a free distributive lattice // Combinatorial Mathematics and Its Applications (Proceedings of Conf., Oxford, 1969). London: Academic Press, 1971. P. 173-181.

[180] О. Б. Лупанов. О принципе локального кодирования и реализации функций из некоторых классов схемами из функциональных элементов // Докл. АН СССР. 1961. Т. 140. № 2. C. $322-325$.

[181] О. Б. Лупанов. О реализации функций алгебры логики формулами из конечных классов (формулами ограниченной глубины) в базисе $\&, \vee,-/ /$ Проблемы кибернетики. Вьп. 6 . М.: Физматгиз, 1961. С. 5-14.

[182] О.Б. Лупанов. О сравнении сложности реализации монотонных функций контактными схемами, содержащими лишь замыкающие контакты, и произвольными схемами // Докл. AH CCCP. 1962. T. 144. № 6. C. 1245-1248.

[183] О. Б. Лупанов. Об одном подходе к синтезу управляющих систем-принципе локалного кодирования // Проблемы кибернетики. Вып. 14. М.: Наука, 1965. С. 31-110.

[184] О. Б. Лупанов. К вопросу о реализации симметрических функций алгебры логики контактными схемами // Проблемы кибернетики.Вып. 15. М.: Наука, 1965. С. 85-99.

[185] О. Б. Лупанов. О влиянии глубины на сложность // Кибернетика. 1970. № 2. С. 46-49. 
[186] О.Б.Лупанов. О методах получения оценок сложности и вычисления индивидуальных функций // Дискретньй анализ: Сб. науч. тр. Вьп. 25. Новосибирск: Ин-т математики CO AH CCCP, 1974. C. 3-18.

[187] О.Б. Лупанов. Асимптотические оценки сложности управляющих систем. М.: МГУ, 1984.

[188] K. Makino, T. Ibaraki. The maximum latency and identification of positive Boolean functions // Algorithms and Computation. 5th International Symposium, ISAAC'94 (Beijing, August 25-27, 1994). Berlin: Springer-Verlag, 1994. P. 324-332. (Lecture Notes in Comput. Sci. V. 834.)

[189] K. Makino, T. Ibaraki. A fast and simple algorithm for identificating 2-monotonic positive Boolean functions // J. Algorithms. 1998. V. 26. № 2. P. 291-305.

[190] С. С. Марченков. Замкнутые классы булевых функций. М.: Физматлит, 2000.

[191] А. А. Марков. О минимальных контактно-вентильных двухполюсниках для монотонных симметрических функций // Проблемы кибернетики. Вып. 8. М.: Физматгиз, 1962. C. $117-121$.

[192] Ал. А. Марков. Введение в теорию кодирования. М.: Наука, 1982.

[193] W. F. McColl. The maximum depth of monotone formulae // Inform. Process. Lett. 1978. V. 7. № 2. P. 65.

[194] W.F. McColl. On the planar monotone computation of threshold functions // STACS'85. Proceedings of the 2nd Annual Symposium on Theoretical Aspects of Computer Science (Saarbrücken, January 3-5, 1985). Berlin: Springer-Verlag, 1985. P. 219-230. (Lecture Notes in Comput. Sci. V. 182.)

[195] K. Mehlhorn. Some remarks on Boolean sums // Acta Informatica. 1979. V. 12. № 4 . P. 371-375. (Рус. пер.: К. Мельхорн. Некоторые замечания, касающиеся булевых сумм // Кибернетический сборник. Нов. сер. Вып. 18. М.: Мир, 1981. С. 39-45.)

[196] K. Mehlhorn, Z. Galil. Monotone switching circuits and Boolean matrix product (extended abstract) // Mathematical Foudations of Computer Science 1975. Proceedings of the Symposium (Mariánské Lázné, 1975). Berlin: Springer-Verlag, 1975. P. 315-319. (Lecture Notes in Comput. Sci. V. 32.)

[197] K. Mehlhorn, Z. Galil. Monotone switching circuits and Boolean matrix product // Computing. 1976. V. 16. № 1-2. P. 99-111.

[198] В. М. Михеев. О множествах, содержащих наиболшшее число попарно несравнимых векторов // Проблемы кибернетики. Вып. 2. М.: Физматгиз, 1959. С. 69-71.

[199] S. Muroga. Lower bounds of the number of threshold functions and a maximum weight // IEEE Trans. Electron. Comput. 1965. V. EC-14. № 2. P. 136-148.

[200] S. Muroga, I. Toda, S. Takasu. Theory of majority decision elements // J. Franklin Inst. 1961. V. 271. № 5. P. 376-418.

[201] S. Muroga, T. Tsuboi, C. H. Baugh. Enumeration of threshold function of eight variables // IEEE Trans. Comput. 1970. V. C-19. № 9. P. 818-825.

[202] Э. И. Нечипорук. О вентильных схемах // Тезисы докладов Международного симпозиума по теории релейных устройств и конечных автоматов (Москва, 24 сентября - 2 октября 1962). M., 1962. C. 42-43.

[203] Э. И. Нечипорук. О синтезе схем из пороговых элементов // Докл. АН СССР. 1964. T. 154. № 4. С. $763-766$.

[204] Э.И. Нечипорук. О синтезе схем из пороговых элементов // Проблемы кибернетики. Вып. 11. М.: Физматгиз, 1964. С. 49-62.

[205] Э.И. Нечипорук. Об одной булевской функции // Докл. АН СССР. 1966. Т. 169. № 4. C. $765-766$.

[206] Э.И. Нечипорук. Об одной булевской матрице // Проблемы кибернетики. Вып. 21. М.: Наука, 1969. С. 237-240.

[207] Э. И. Нечипорук. О реализации дизъюнкции и конъюнкции в некоторых монотонных базисах // Проблемы кибернетики. Вып. 23. М.: Наука, 1970. С. 291-293.

[208] Р. Г. Нигматуллин. Вариационный принцип в алгебре логики // Дискретный анализ: Сб. науч. тр. Вып. 10. Новосибирск: Ин-т математики СО АН СССР, 1969. С. 69-89. 
[209] Р. Г. Нигматуллин. Нижние оценки сложности и сложность универсальных схем. Казань: Изд-во Казанского ун-та, 1990.

[210] Р. Г. Нигматуллин. Сложность булевых функций. М.: Наука, 1991.

[211] I. Ninomiya. A study of the structures of Boolean functions and its application to the synthesis of switching circuits // Mem. Fac. Eng. Nagoya Univ. 1961. V. 13. № 2.

[212] С. В. Новиков, В.Э. Комиссаров, В.П. Супрун. Минимальная реализация функции $f=x_{1} \ldots x_{r}$ схемами из элементов шефферовского типа // Вестник Белорусского ун-та. Сер. 1. 1975. №2. С. 13-17.

[213] Н. Н. Нурмеев. О сложности схемной реализации почти всех монотонных булевых функций // Изв. вузов. Матем. 1981. № 5. С. 64-70.

[214] Е. А. Окольнишникова. О роли отрицаний при реализации монотонных булевых функций формулами в базисе $(\vee, \&,-) / /$ Методы дискретного анализа в решении экстремальных задач: Сб. науч. тр. Вып. 33. Новосибирск: Ин-т математики СО АН СССР, 1979. C. $68-76$.

[215] Е. А. Окольнишникова. О влиянии отрицаний на сложность реализации монотонных булевых функций формулами ограниченной глубины // Методы дискретного анализа в оценках сложности управляющих систем: Сб. науч. тр. Вып. 38. Новосибирск: Ин-т математики СО АН СССР, 1982. С. 74-80.

[216] Е. А. Окольнишникова. Монотонная булева система с квадратичной сложностью реализации в базисе $\{\vee, \&, 0,1\} / /$ Методы дискретного анализа в изучении реализаций логических функций: Сб. науч. тр. Вып. 41. Новосибирск: Ин-т математики СО АН СССР, 1984. C. 81-98.

[217] M.S. Paterson. Complexity of monotone networks for Boolean matrix product // Theoret. Comput. Sci. 1975. V. 1. №1. P. 13-20. (Рус. пер.: М. С. Патерсон. Сложность монотонных схем для булева умножения матриц // Кибернетический сборник. Нов. сер. Вып. 15. М.: Мир, 1978. С. 28-37.)

[218] M.S. Paterson. New bounds on formula size // Theoretical Computer Science. Third GI conference (Darmstadt, 1977). Berlin: Springer-Verlag, 1977. P. 17-26. (Lecture Notes in Comput. Sci. V. 48.)

[219] W. J. Paul. Realizing Boolean functions on disjoint sets of variables // Theoret. Comput. Sci. 1976. V. 2. № 3. P. 383-396.

[220] N. Pippenger. The realization of monotone Boolean functions (preliminary version) // Proceedings of the Eighth Annual ACM Symposium on Theory of Computing (Hershey, 1976). New York: ACM Press, 1976. P. 204-210.

[221] N. Pippenger. The complexity of monotone Boolean functions // Math. Systems Theory. 1977/78. V. 11. № 4. P. 289-316.

[222] N. Pippenger. On another Boolean matrix // Theoret. Comput. Sci. 1980. V. 11. № 1. P. $49-56$.

[223] N. Pippenger. Entropy and enumeration of Boolean functions // IEEE Trans. Inform. Theory. 1999. V. 45. № 6. P. 2096-2100.

[224] L. Pitas, A. N. Venetsanopoulos. Nonlinear Digital Filters: Principles and Applications. Boston: Kluwer, 1980.

[225] В. Я. Пивкин. О сравнении сложности реализации монотонных функций формулами в базисах $(\vee, \&)$ и $(\vee, \&,-) / /$ Проблемы кибернетики. Вып. 15. М.: Наука, 1965. С. 275-279.

[226] E. L. Post. The Two-Valued Iterative Systems of Mathematical Logic. Princeton: Princeton Univ. Press, 1941. (Ann. of Math. Stud. V. 5.)

[227] V. R. Pratt. The power of negative thinking in multiplying Boolean matrices // Proceedings of the Sixth Annual ACM Symposium on Theory of Computing (Seattle, 1974). New York: ACM Press, 1974. P. 80-83.

[228] V. R. Pratt. The power of negative thinking in multiplying Boolean matrices // SIAM J. Comput. 1975. V. 4. № 3. P. 326-330.

[229] P. Pudlák. Bounds for Hodes-Specker theorem // Logic and Machines: Decision Problems and Complexity (Münster, 1983). Berlin: Springer-Verlag, 1984. P. 421-445. (Lecture Notes in Comput. Sci. V. 171.) 
[230] J. Radhakrishnan. Better bounds for threshold formulas // Proceedings of the 32nd Annual Symposium on Foundations of Computer Science (San Juan, October 1-4, 1991). Los Alamitos: IEEE Comput. Soc. Press, 1991. P. 314-323.

[231] J. Radhakrishnan. $\Sigma \Pi \Sigma$ threshold formulas // Combinatorica. 1994. V. 14. № 3. P. 345-374.

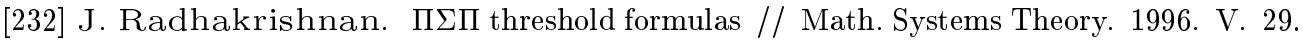
№ 4. P. 357-374.

[233] J. Radhakrishnan. Better lower bounds for monotone threshold formulas // J. Comput. System Sci. 1997. V. 54. № 2. Part 1. P. 221-226.

[234] J. Radhakrishnan, M. M. Halldórsson, K. V. Subrahmanyan. Directed monotone contact networks for threshold functions // Inform. Process. Lett. 1994. V. 50. № 4. P. 199-203.

[235] R. Raz, P. McKenzie. Separation of the monotone NC hierarchy // Proceedings of the 38th Symposium on Foundations of Computer Science (Miami Beach, October 1997). Los Alamitos: IEEE Comput. Soc. Press, 1997. P. 234-243.

[236] R. Raz, P. McKenzie. Separation of the monotone NC hierarchy // Combinatorica. 1999. V. 19. № 3. P. 403-435.

[237] R. Raz, A. Wigderson. Monotone circuits for matching require linear depth // Proceedings of the 22nd Annual ACM Symposium on Theory of Computing (Baltimore, 1990). New York: ACM Press, 1990. P. 287-292.

[238] R. Raz, A. Wigderson. Monotone circuits for matching require linear depth // J. ACM. 1992. V. 39. № 3. P. 736-744.

[239] А. А. Разборов. Нижние оценки монотонной сложности некоторых булевых функций // Докл. АН СССР. 1985. Т. 281. № 4. С. 798-801.

[240] А. А. Разборов. Нижние оценки монотонной сложности логического перманента // Матем. заметки. 1985. Т. 37. №6. С. 887-900.

[241] А. А. Разборов. Нижние оценки размера схем ограниченной глубины в базисе $\{\&, \vee, \oplus\}$ // УМН. 1986. Т. 41. № 4. С. 213-220.

[242] А. А. Разборов. Нижние оценки размера схем ограниченной глубины в полном базисе, содержащем функции логического сложения // Матем. заметки. 1987. Т. 41. № 4 . C. 598-607.

[243] А. А. Разборов. Нижние оценки сложности булевых функций // Proceedings of the International Congress of Mathematicians (Berkeley, 1986). V. 2. Providence, RI: Amer. Math. Soc., 1987. P. 1478-1487.

[244] A. A. Razborov. On the method of approximations // Proceedings of the 21st Annual ACM Symposium on the Theory of Computing (Seattle, May 15-17, 1989). New York: ACM Press, 1989. P. 167-176.

[245] A. A. Razborov. Applications of matrix methods to the theory of lower bounds in computational complexity // Combinatorica. 1990. V. 10. № 1. P. 81-93.

[246] A. A. Razborov. On small depth threshold circuits // Algorithm Theory - SWAT'92. Proceedings of the Third Scandinavian Workshop on Algorithm Theory (Helsinki, July 8-10, 1992). Berlin: Springer-Verlag, 1992. P. 42-52. (Lecture Notes in Comput. Sci. V. 621.)

[247] A. A. Razborov. On submodular complexity measures // Boolean Function Complexity (Durham, 1990). Cambridge: Cambridge Univ. Pres, 1992. P. 76-83. (London Math. Soc. Lecture Note Ser. V. 169.)

[248] A. A. Razborov. Bounded arithmetic and lower bounds in Boolean complexity // Feasible Mathematics, II (Ithaca, 1992). Boston: Birkhäuser, 1995. P. 344-386. (Progr. Comput. Sci. Appl. Logic. V. 13.)

[249] A. A. Razborov, S. Rudich. Natural proofs // Proceedings of the 26th Annual ACM Symposium on Theory of Computing (Montréal, May 23-25, 1994). New York: ACM Press, 1994. P. 204-213.

[250] A. A. Razborov, S. Rudich. Natural proofs // J. Comput. System Sci. 1997. V. 55. № 1. Part 1. P. 24-35. 
[251] Н. П. Редькин. О реализации монотонных булевых функций контактными схемами // Проблемы кибернетики. Вып. 35. М.: Наука, 1979. С. 87-110.

[252] Н. П. Редькин. Асимптотически минимальные самокорректирующиеся схемы для одной последовательности булевьх функций // Дискрет. анализ и исслед. операций. 1996. Т. 3. № 2. C. $62-79$.

[253] Н. П. Редькин. Минимальные самокорректирующиеся схемы для одной последовательности булевых функций // Дискрет. анализ и исслед. операций. Сер. 1. 1998. Т. 5. № 3. C. $44-63$.

[254] В. И. Резник. О реализации монотонных функций схемами из функциональных элементов // Докл. АН СССР. 1961. Т. 139. № 3. С. 566-569.

[255] J. Riordan, C. Shannon. The number of two-terminal series-parallel networks // J. Math. Phys. 1942. V. 21. № 2. Р. 83-93. (Рус. пер.: Дж. Риордан, К. Шеннон. Число двухполюсных параллельно-последовательных сетей // К. Шеннон. Работы по теории информации и кибернетике. М.: ИЛ, 1963. С. 46-58.)

[256] R. L. Rivest. The necessity of feedback in minimal monotone combinatorial circuits // IEEE Trans. Comput. 1977. V. C-26. №6. P. 606-607.

[257] N. M. Riviere. Recursive formulas on free distributive lattices // J. Combin. Theory. 1968. V. 5. № 3. P. 229-234.

[258] A. Rosenbloom. Monotone real circuits are more powerful than monotone Boolean circuits // Inform. Process. Lett. 1997. V. 61. № 3. P. 161-164.

[259] А. А. Сапоженко. О числе связных подмножеств с заданной мощностью границы в двудольном графе // Методы дискретного анализа в решении комбинаторных задач: Сб. науч. тр. Вып. 45. Новосибирск: Ин-т математики СО АН СССР, 1987. С. 42-70.

[260] А. А. Сапоженко. Асимптотика числа монотонных функций на частично упорядоченных множествах // Докл. АН СССР. 1989. Т. 305. № 2. С. 279-283.

[261] А. А. Сапоженко. О числе антицепей в ранжированных частично упорядоченных множествах // Дискрет. матем. 1989. Т. 1. № 1. С. 74-93.

[262] А. А. Сапоженко. О числе антицепей в многослойных ранжированных множествах // Дискрет. матем. 1989. Т. 1. № 2. С. 110-128.

[263] А. А. Сапожкенко. О поиске максимального верхнего нуля монотонных функций на ранжированных множествах // Журн. вьгисл. матем. и матем. физ. 1991. Т. 31. № 12. C. $1871-1884$.

[264] А. А. Сапоженко. Оценка числа связных компонент в графе и структура компонент случайных подмножеств // Докл. АН СССР. 1996. Т. 365. № 4. С. 455-457.

[265] А. А. Сапоженко. О числе связных множеств с заданной мощностью границы // Дискрет. анализ и исслед. операций. Сер. 1. 1997. Т. 4. № 3. С. 18-34.

[266] А. А. Сапоженко. Метод граничных функционалов в перечислительных изопериметрических задачах // Сборник трудов семинара по дискретной математике и ее приложениям (Москва, 2-4 февраля 1998). М.: Изд-во мех.-матем. фак-та МГУ, 1998. С. 43-49.

[267] А. А. Сапоженко. Проблема Дедекинда и метод граничных функционалов // Математические вопросы кибернетики. Вып. 9. М.: Физматлит, 2000. С. 161-220.

[268] J. E. Savage. The Complexity of Computing. New York: Wiley, 1976. (Рус. пер.: Д.Э. Сэвидж. Сложность вычислений. М.: Факториал, 1998.)

[269] C.P. Schnorr. A lower bound on the number of additions in monotone computation // Theoret. Comput. Sci. 1976. V.2. № 3. Р. 305-315. (Рус. пер.: К.П. Шнорр. Нижняя оценка числа сложений в монотонных вычислениях // Кибернетический сборник. Нов. сер. Вып. 18. М.: Мир, 1982. С. 5-20.)

[270] C.P. Schnorr. Computation of the Boolean matrix-vector, AND/OR-product in average time $O(m+n \ln n) / /$ Informatik. Stuttgart: Teubner, 1992. P. 359-362. (Teubner-Texte Inform. V. 1.)

[271] U. Schürfeld. New lower bounds on the formula size of Boolean functions // Acta Inform. 1983. V. 19. № 2. P. 183-194.

[272] А. В. Сержантов. Оптимальный алгоритм расшифровки некоторых классов монотонных функций // Журн. вычисл. матем. и матем. физ. 1983. Т. 23. № 1. С. 206-212. 
[273] А. В. Сержантов. Об оптимальном алгоритме расшифровки монотонных функций конечнозначной логики // Математические вопросы кибернетики. Вып. 1. М.: Наука, 1988. C. 223-233.

[274] О. В. Шабанин. О сложности дизъюнктивной нормальной формы пороговых функций // Дискрет. матем. 2000. Т. 12. № 2. С. 85-92.

[275] C. E. Shannon. A symbolic analysis of relay and switching circuits // Trans. AIEE. 1938. V. 57. Р. 713-722. (Рус. пер.: К. Шеннон. Работы по теории информации и кибернетике. М.: ИЛ, 1963. С. 9-45.)

[276] C. E. Shannon. The synthesis of two-terminal switching circuits // Bell System. Tech. J. 1949. V. 28. № 1. Р. 59-98. (Рус. пер.: К. Шеннон. Работы по теории информации и кибернетике. М.: ИЛ, 1963. С. 59-101.)

[277] H. N. Shapiro. On the counting problem for monotone Boolean functions // Comm. Pure Appl. Math. 1970. V. 23. № 3. P. 299-312.

[278] I. Shmulevich, A. D. Korshunov, J. Astola. Almost all monotone Boolean functions are polynomially learnable using membership queries // Inform. Process. Lett. 2001. V. 79. № 5. P. 211-213.

[279] I. Shmulevich, O. Yli-Harja, J. Astola, A. Korshunov. On the robustness of the class of stack filters // IEEE Trans. Signal Process. 2002. V. 50. № 7. P. 1640-1649.

[280] С. Е. Шумилина. Комбинаторно-алгебраические методы и их применение. Горький: Горьк. гос. ун-т, 1987.

[281] S. Skyum. A measure in which Boolean negation is exponentially powerful // Inform. Process. Lett. 1983. V. 17. № 3. P. 125-128.

[282] R. Smolensky. Algebraic methods in the theory of lower bounds for Boolean circuit complexity // Proceedings of the 19th Annual ACM Symposium on Theory of Computing (New York, 1987). New York: ACM Press, 1987. P. 77-86.

[283] Н. А. Соколов. Поиск максималшного верхнего нуля для одного класса монотонных дискретных функций // Докл. АН СССР. 1980. Т. 251. № 5. С. 1077-1080.

[284] Н. А. Соколов. Об оптимальной расшифровке монотонных функций алгебры логики // Журн. вычисл. матем. и матем. физ. 1982. Т. 22. № 2. С. 449-461.

[285] Н. А. Соколов. Частичная расшифровка монотонных булевых функций // Журн. вычисл. матем. и матем. физ. 1983. Т. 23. № 5. С. 1267-1271.

[286] Н. А. Соколов. Оптимальная расшифровка монотонных булевых функций // Журн. вьчисл. матем. и матем. физ. 1987. Т. 27. № 12. С. 1878-1887.

[287] Н. А. Соколов. Частичные порядки и порядковая оптимизация // Методы комбинаторной оптимизации. М.: ВЦ РАН, 1997. С. 68-86.

[288] Н. А. Соколов. Об одной задаче расшифровки монотонных булевых функций // Комбинаторные модели и методы. Вьп. 2. М.: ВЦ РАН, 1997. С. 73-84.

[289] Е. П. Сопруненко. О минимальной реализации некоторых функций схемами из функционалшных элементов // Проблемы кибернетики. Вып. 15. М.: Наука, 1965. С. 117-134.

[290] E. Sperner. Ein Satz über Untermengen einer endlichen Menge // Math. Z. 1928. V. 27. № 4. P. 544-548.

[291] V. Strassen. Gaussian elimination is not optimal // Numer. Math. 1969. V. 13. № 4. Р. 354-356. (Рус. пер.: В. Штрассен. Алгоритм Гаусса не оптимален // Кибернетический сборник. Нов. сер. Вып. 7. М.: Мир, 1970. С. 67-70.)

[292] Н. Н. Субоч. Реализация монотонных функций алгебры логики контактными $\pi$-схемами // Проблемы кибернетики. Вып. 17. М.: Наука, 1966. С. 247-253.

[293] Н. Н. Субоч. Реализация монотонных функций с неболшшим числом конъюнкций в сокращенной д.н.ф. схемами из замькающих контактов // Дискретный анализ: Сб. науч. тр. Вып. 15. Новосибирск: Ин-т математики СО АН СССР, 1969. С. 66-78.

[294] Б. А. Субботовская. О реализации линейных функций формулами в базисе $\{\&, \vee,-\}$ // Докл. АН СССР. 1961. Т. 136. № 3. С. 553-555.

[295] É. Tardos. The gap between monotone and non-monotone circuit complexity is exponential // Combinatorica. 1988. V. 8. № 1. P. 141-142. 
[296] R.E. Tarjan. Complexity of monotone networks for computing conjunctions // Algorithmic Aspects of Combinatorics (Vancouver, 1976). Amsterdam: North-Holland, 1978. P. 121-133. (Ann. Discrete Math. V. 2.)

[297] В. И. Теренков. О точности алгоритмов вычисления оценок для таблиц, порождаемшх монотонными булевыми функциями // Журн. вычисл. матем. и матем. физ. 1973. Т. 13. №6. C. $1620-1625$.

[298] J. Tiekenheinrich. A $4 n$-lower bound on the monotone network complexity of a one-output Boolean function // Inform. Process. Lett. 1984. V. 18. № 4. P. 201-202.

[299] Г. П. Тоноян. Разбиение вершин $n$-мерного куба на цепи и расшифрровка монотонных булевых функций // Журн. вычисл. матем. и матем. физ. 1979. Т. 19. №6. С. 1532-1542.

[300] Г. П. Тоноян. Некоторые характеристики одного алгоритма расшифровки монотонных булевых функций // Молодой научный сотрудник. Естеств. науки. № 2/32, 1980. С. 85-87.

[301] В. Н. Тришин. Адаптивный алгоритм для решения многомерной задачи о ранце и распознавания монотонной булевой функции // Изв. АН СССР. Сер. техн. кибернетика. 1982. № 4. C. 11-18.

[302] А. Б. Угольников. О реализации монотонных функций схемами из функционалшных элементов // Проблемы кибернетики. Вып. 31. М.: Наука, 1976. С. 167-185.

[303] А. Б. Угольников. О реализации функций из замкнутых классов схемами из функциональных элементов в полном базисе // Докл. АН СССР. 1983. Т. 271. № 1. С. 49-51.

[304] А. Б. Угольников. О реализации функций из замкнутых классов схемами из функциональных элементов // Математические вопросы кибернетики. Вып. 1. М.: Наука, 1988. C. $89-113$.

[305] А. Б. Угольников. О глубине формул в неполных базисах // Математические вопросы кибернетики. Вып. 1. М.: Наука, 1988. С. 242-245.

[306] А. Б. Угольников. Сложность функций из замкнутых классов // Сборник трудов семинара по дискретной математике и ее приложениям. М.: Изд-во мех.-матем. ф-та МГУ, 1998. C. $49-56$.

[307] L. G. Valiant. Exponential lower bounds for restricted monotone circuits // Proceedings of the 15th Annual ACM Symposium on Theory of Computing (Boston, 1983). New York: ACM Press, 1983. P. 110-117.

[308] L. G. Valiant. Short monotone formulae for the majority function // J. Algorithms. 1984. V. 5. № 3. Р. 363-366. (Рус. пер.: Л. Вэльянт. Простые монотонные формулы для функции голосования // Кибернетический сборник. Нов. сер. Вьп. 24. М.: Мир, 1987. С. 97-100.)

[309] L. G. Valiant. Negation is powerless for Boolean slice functions // SIAM J. Comput. 1986. V. 15. № 2. Р. 531-535. (Рус. пер.: Л. Дж. Вальянт. Отрицание малоэффективно для булевых слой-функций // Кибернетический сборник. Нов. сер. Вьп. 27. М.: Мир, 1990. C. $97-103$.

[310] В.А. Варданян. О сложности динамических тестов для монотонных булевых функций // Докл. АН АрмССР. 1985. Т. 80. № 4. С. 147-151.

[311] V. A. Vardanian. On the length of single dynamic tests for monotone Boolean functions // Foundamentals of Computation Theory. FCT'85 (Cottbus, September 9-13, 1985). Berlin: Springer-Verlag, 1985. P. 442-449. (Lecture Notes in Comput. Sci. V. 199.)

[312] В. А. Варданян. О сложности единичных динамических тестов для монотонных булевых функций // Кибернетика. 1987. № 3. С. 23-26.

[313] В.А. Варданян. О сложности полных проверяющих тестов для монотонных булевых функций // Докл. АН Армении. 1993. Т. 94. № 2. С. 93-97.

[314] Ю. Л. Васильев. Минимальные контактные схемы для булевых функций четырех переменных // Докл. АН СССР. 1959. V. 127. № 2. Р. 242-245.

[315] А. А. Вороненко. О сложности распознавания монотонности // Математические вопросы кибернетики. Вып. 8. М.: Наука, 1999. С. 301-303.

[316] M. Ward. Note on the order of free distributive lattices // Bull. Amer. Math. Soc. 1946. V. 52. № 5. P. 423. 
[317] I. Wegener. Switching functions whose monotone complexity is nearly quadratic // Conference Record of the 10th Annual ACM Symposium on Theory of Computing (San Diego, 1978). New York: ACM Press, 1978. P. 143-149.

[318] I. Wegener. Switching functions whose monotone complexity is nearly quadratic // Theoret. Comput. Sci. 1979. V. 9. № 1. Р. 83-97. (Рус. пер.: И. Вегенер. Булевы функции, чья монотонная сложность почти квадратична / / Кибернетический сборник. Нов. сер. Вып. 18. М.: Мир, 1982. С. 55-74.)

[319] I. Wegener. A counterexample to a conjecture of Schnorr referring to monotone networks // Theoret. Comput. Sci. 1979. V. 9. №1. P. 147-150.

[320] I. Wegener. A new lower bound on the monotone network complexity of Boolean sums // Acta Informatica. 1980. V. 13. № 2. P. 109-114.

[321] I. Wegener. Boolean functions whose monotone complexity is of size $n^{2} / \log n$ // Theoretical Computer Science. 5th GI-conference (Karlsruhe, March 23-25, 1981). Berlin: Springer-Verlag, 1981. P. 22-31. (Lecture Notes in Comput. Sci. V. 104.)

[322] I. Wegener. Best possible asymptotic bounds on the depth of monotone functions in multivalued logic // Inform. Process. Lett. 1982. V. 15. № 2. P. 81-83.

[323] I. Wegener. Boolean functions whose monotone complexity is of size $n^{2} / \log n / /$ Theoret. Comput. Sci. 1982. V. 21. № 2. Р. 213-224. (Рус. пер.: И. Вегенер. Булевы функции, чья монотонная сложность имеет величину порядка $n^{2} / \log n / /$ Кибернетический сборник. Нов. сер. Вып. 21. М.: Мир, 1984. С. 69-84.)

[324] I. Wegener. Relating monotone formula size and monotone depth of Boolean functions // Inform. Process. Lett. 1983. V. 16. № 1. P. 41-42.

[325] I. Wegener. Proving lower bounds on the monotone complexity of Boolean functions // Logic and Machines: Decision Problems and Complexity. Proceedings of the Symposium (Münster/Westfalen, May 23-28, 1983). Berlin: Springer-Verlag, 1984. P. 446-456. (Lecture Notes in Comput. Sci. V. 171.)

[326] I. Wegener. On the complexity of slice functions // Mathematical Foundations of Computer Science (Prague, 1984). Berlin: Springer-Verlag, 1984. P. 553-561. (Lecture Notes in Comput. Sci. V. 176.)

[327] I. Wegener. The critical complexity of all (monotone) Boolean functions and monotone graph properties // Foundamentals of Computation Theory (Cottbus, September 9-13, 1985). Berlin: Springer-Verlag, 1985. P. 494-502. (Lecture Notes in Comput. Sci. V. 199.)

[328] I. Wegener. On the complexity of slice functions // Theoret. Comput. Sci. 1985. V. 38. № 1. P. $55-68$.

[329] I. Wegener. The Complexity of Boolean Functions. Chichester/Stuttgart: Wiley/Teubner, 1987.

[330] I. Wegener, N. Wurm, S.-Z. Yi. Symmetric functions in $\mathrm{AC}^{0}$ can be computed in constant depth with very small size // Boolean Function Complexity (Durhan, 1990). Cambridge: Cambridge Univ. Press, 1992. P. 129-139. (London Math. Soc. Lecture Note Ser. V. 169.)

[331] J. Weiss. An $n^{3 / 2}$ lower bound on the monotone network complexity of the Boolean convolution // Inform. and Control. 1983. V. 59. № 1-3. P. 184-188.

[332] P. Wendt, E. Coyle, N. Gallager. Stack filters // IEEE Trans. Acoust. Speech Signal Process. 1986. V. ASSP-34. P. 898-911.

[333] D. Wiedemann. A computation of the eighth Dedekind number // Order. 1991. V. 8. № 1. P. 5-6.

[334] R. O. Winder. Single stage threshold logic // Switching Circuit Theory and Logical Design. AIEE Special Publ. 1960. P. 321-322.

[335] R. O. Winder. Enumeration of seven-argument threshold functions // IEEE Trans. Electron. Comput. 1965. V. EC-14. № 3. P. 315-325.

[336] С.В.Яблонский. О классах функций алгебры логики, допускающих простую схемную реализацию // УМН. 1957. Т. 12. №6. С. 189-196. 
[337] С. В. Яблонский. Об алгоритмических трудностях синтеза минималњных контактных схем // Проблемы кибернетики. Вып. 2. М.: Физматгиз, 1957. С. 75-121.

[338] С. В. Яблонский, Г. П. Гаврилов, В.Б.Кудрявцев. Функции алгебры логики и классы Поста. М.: Наука, 1966.

[339] S. Yajima, T. Ibaraki. A lower bound of the number of threshold functions // IEEE Trans. Electron. Comput. 1965. V. EC-14. №6. Р. 926-929. (Рус. пер.: С. Яджима, Т. Ибараки. Нижняя оценка числа пороговых функций // Кибернетический сборник. Нов. сер. Вып. 6. М.: Мир, 1969. С. 72-81.)

[340] K. Yamamoto. Logarithmic order of free distributive lattice // J. Math. Soc. Japan. 1954. V. 6. P. 343-353.

[341] A. C.-C. Yao. Separating the polynomial-time hierarchy by oracles // Proceedings of the 26th Annual Symposium on Foundations of Computer Science. Los Alamitos: IEEE Comput. Soc. Press, 1985. P. 1-10.

[342] A. C.-C. Yao. Circuits and local computation // Proceedings of the 21st Annual ACM Symposium on Theory of Computing (Seattle, 1989). New York: ACM Press, 1989. P. $186-196$.

[343] A. C.-C. Yao. A lower bound for the monotone depth of connectivity // Proceedings of the 35th Annual Symposium on Foundations of Computer Science (Santa Fe, 1994). Los Alamitos: IEEE Comput. Soc. Press, 1994. P. 302-308.

[344] L. Yin, R. Yang, M. Gabbouj, Y. Neuvo. Weighted median filters: a tutorial // IEEE Trans. Circuits Systems. 1996. V. 43. № 3. P. 157-192.

[345] А. И. Зеличенко. О соответствии монотонных булевых функций системам линейных неравенств // Журн. вычисл. матем. и матем. физ. 1979. Т. 19. №6. С. 1543-1554.

[346] Ю. И. ЖКуравлев. Об алгоритмах выделения совокупности существенных переменных не всюду определенных функций алгебры логики // Проблемы кибернетики. Вып. 11. М.: Наука, 1964. С. 271-275.

[347] Ю. И. Ж Куравлев, С. Н. Мирошник, С. М. Швартин. Об одном подходе к оптимизации в классе параметрических алгоритмов распознавания // Журн. вычисл. матем. и матем. физ. 1976. Т. 16. №1. С. 209-218.

[348] Ю. А. Зуев. О представлении булевых функций системами линейных неравенств // Кибернетика. 1985. № 5. С. 7-9.

[349] Ю. А. Зуев. Асимптотика логарифма числа пороговых функций алгебры логики // Докл. АН СССР. 1989. Т. 306. № 3. С. 528-530.

[350] Ю. А. Зуев. Комбинаторно-вероятностные и геометрические методы в пороговой логике // Дискрет. матем. 1991. Т. 3. № 2. С. 47-57.

[351] Ю. А. Зуев. Пороговые функции и пороговое представление булевых функций // Математические вопросы кибернетики. Вып. 5. М.: Физматгиз, 1994. С. 5-61.

[352] Ю. А. Зуев, Л.И.Липкин. О числе линейных отделимых булевых множеств заданной мощности // Методы дискретного анализа в теории графов и логических функций: Сб. науч. тр. Вып. 43. Новосибирск: Ин-т математики СО АН СССР, 1986. С. 29-39.

[353] Ю. А. Зуев, Л.И.Липкин. К оценке эффективности пороговых представлений булевых функций // Кибернетика. 1988. №6. С. 29-37.

[354] Ю.А. Зуев, Л.И. Липкин. Линейные отсечения заданной мощности в единичном гиперкубе // Изв. АН СССР. Сер. техн. кибернетика. 1988. № 3. С. 79-85.

[355] Ю. А. Зуев, В.Н. Тришин. Нижняя оценка числа неравенств, представляющих монотонную булеву функцию от $n$ переменных // Журн. выгисл. матем. и матем. физ. 1983. T. 23. № 3. C. $754-756$.

[356] Ю. А. Зуев, В.Н. Тришин. О связи линейных неравенств с монотонными булевыми функциями // Журн. вычисл. матем. и матем. физ. 1984. Т. 24. № 5. С. 780-781. 